\title{
Computational characterization of adhesive bond properties using guided waves in bonded plates
}

\author{
A Thesis \\ Presented to \\ The Academic Faculty \\ by \\ Juergen Koreck
}

\author{
In Partial Fulfillment \\ of the Requirements for the Degree \\ Master of Science in Engineering Science and Mechanics
}

School of Civil and Environmental Engineering

Georgia Institute of Technology

December 2006 


\section{Computational characterization of adhesive bond properties using guided waves in bonded plates}

Approved by:

Dr. Laurence Jacobs, Advisor

School of Civil and Environmental

Engineering

Georgia Institute of Technology

Dr. Jianmin Qu

George W. Woodruff School of Mechanical Engineering

Georgia Institute of Technology

Dr. Christine Valle

George W. Woodruff School of

Mechanical Engineering

Georgia Institute of Technology

Date Approved: August 25, 2006 


\section{ACKNOWLEDGEMENTS}

My special gratitude goes to my advisors Dr. Laurence J. Jacobs and Dr. Christine Valle. I would like to thank Dr. Jacobs for being an excellent advisor and friend. I am grateful for all support I received from him during my year at Georgia Tech. I very appreciate that he made it possible for us to present the results of this research on the 2006 QNDE conference in Portland, Oregon.

I would like to thank Dr. Christine Valle for her advice and help not only with the finite element simulation. I am very grateful for all her suggestions and all the interesting discussions.

I would also like to thank Dr. Jianmin Qu from the Woodruff School of Mechanical Engineering for his help and support.

Furthermore, I would like to express my gratitude to Prof. Lothar Gaul from the University of Stuttgart for giving me the opportunity to study at Georgia Tech. I would also like to thank the DAAD (German Academic Exchange Service) for providing generous support for my studies in the U.S. 


\section{TABLE OF CONTENTS}

ACKNOWLEDGEMENTS .................. . . iii

LIST OF TABLES . . . . . . . . . . . . . . . . . vi

LIST OF FIGURES ...................... . . . . . . . . . . . .

SUMMARY . . . . . . . . . . . . . . . . . . 1

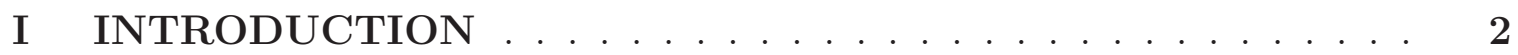

II THEORETICAL BACKGROUND OF WAVE PROPAGATION 4

2.1 Governing equations in linear elasticity . . . . . . . . . . . . . 4

2.2 Wave phenomena .................. 6

2.3 Reflection of body waves . . . . . . . . . . . . . . . . . 7

2.4 Guided waves . . . . . . . . . . . . . . . . . 9

III METHODS FOR LAYERED MEDIA . . . . . . . . . . . . . 13

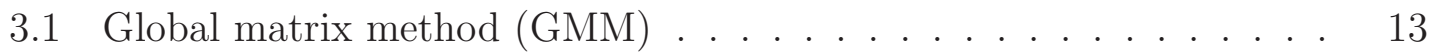

3.1.1 Equation of motion - Displacement . . . . . . . . . . . 14

3.1 .2 Strain and Stress . . . . . . . . . . . . . . . 16

3.1.3 Boundary conditions ................. 17

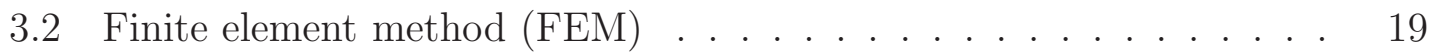

3.2.1 FE theory . . . . . . . . . . . . . . . . . 19

3.2.2 FE program ...................... 20

3.2.3 FE criteria . . . . . . . . . . . . . . . . . 20

3.2 .4 Nyquist criteria . . . . . . . . . . . . . . . . 21

3.2.5 FE model . . . . . . . . . . . . . . . . . 22

3.2.6 Signal processing . . . . . . . . . . . . . 25

3.3 Waveguide FE method (WFE) . . . . . . . . . . . . . 27

3.3.1 Dynamic Stiffness Matrix . . . . . . . . . . . . . 27

3.3.2 Transfer matrix . . . . . . . . . . . . . . . . 28

3.3.3 Dispersion relation . . . . . . . . . . . . . . . . 29 
3.3 .4 Example . . . . . . . . . . . . . . . . 30

IV FORWARD PROBLEM . . . . . . . . . . . . . . . . . 33

4.1 Dispersion relations for single and double layered plates (GMM) . . 34

4.2 Typical FE results . . . . . . . . . . . . . . . . . . . . . . 34

4.3 Parametric studies $(\mathrm{GMM}) \ldots \ldots \ldots$

4.3.1 Variation of the adhesive bond thickness . . . . . . . 39

4.3.2 Variation of the Young's modulus . . . . . . . . . . . . 41

4.3.3 Variation of the Poisson's ratio . . . . . . . . . . . . . . 41

4.4 Typical waveguide FE (WFE) results . . . . . . . . . . . 44

V CONNECTING FORWARD TO INVERSE PROBLEM . . . . 46

5.1 Frequency-wavenumber representation . . . . . . . . . . 46

5.2 Slowness-frequency representation . . . . . . . . . . . . 47

5.3 Adhesive bond parameter influence . . . . . . . . . . . . . . . 49

VI INVERSE PROBLEM . . . . . . . . . . . . . . . . 51

6.1 Parts of the inversion process . . . . . . . . . . . . . 52

6.1.1 Computing the sensitive points with the GMM . . . . . . 52

6.1 .2 Error-function $e \ldots \ldots \ldots \ldots \ldots \ldots \ldots$

6.1 .3 Optimization algorithm . . . . . . . . . . . 54

6.1.4 Initial guess, lower and upper bounds . . . . . . . . . 55

6.2 Results . . . . . . . . . . . . . . . . . . . 5 56

6.2.1 Case of only one transient output signal . . . . . . . 57

6.2.2 Case of multiple transient output signals . . . . . . . 58

6.3 Limits and outlook . . . . . . . . . . . . . . . . 60

6.3.1 Lower stiffness adhesive bond . . . . . . . . . . . . 60

6.3 .2 Triple layered plate . . . . . . . . . . . . . . . 61

VII CONCLUSION $\ldots \ldots \ldots \ldots \ldots \ldots \ldots \ldots$

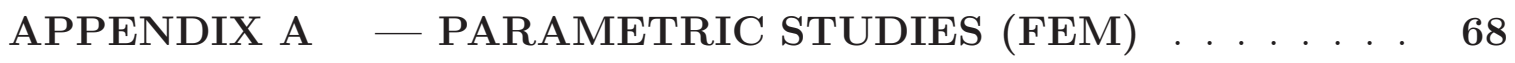

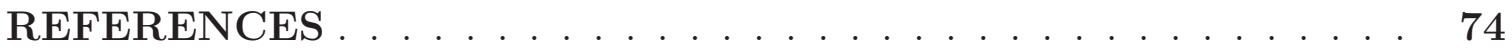




\section{LIST OF TABLES}

Table 3.1 Low and high resolution FE model settings (rounded values). . . 25

Table $4.1 \quad$ Material properties. . . . . . . . . . . . . . . . . . 33

Table 6.1 Starting points, lower and upper bounds for the inversion. . . . . 56

Table $6.2 \quad$ Frequency locations of the critical points. . . . . . . . . . . 57 


\section{LIST OF FIGURES}

Figure 2.1 Particle motion of body waves . . . . . . . . . . . . . . 6

Figure 2.2 Reflection of body waves. . . . . . . . . . . . . . . . . . . . . 8

Figure 2.3 Reflections in a waveguide. . . . . . . . . . . . . . . . . 9

Figure 2.4 Dispersion relation in the frequency-wavenumber domain. . . . . 11

Figure 2.5 Dispersion relation in the slowness-frequency domain. . . . . . . 12

Figure 3.1 Double layer model for the GMM. . . . . . . . . . . . . . . . 13

Figure 3.2 High resolution FE model. . . . . . . . . . . . . . . . . . . . . . 24

Figure 3.3 Low resolution FE model. . . . . . . . . . . . . . . . . . . . . . . 24

Figure 3.4 FE mesh of three sections in the waveguide. . . . . . . . . . . . . 28

Figure 3.58 element and 20 element models. . . . . . . . . . . . . . . . . 31

Figure 3.6 Waveguide FE results for a 8 element and a 20 element model of a $1 \mathrm{~mm}$ aluminum plate. . . . . . . . . . . . . 32

Figure 4.1 GMM for Alu, Tape2 and Alu-Tape2. . . . . . . . . . . . . . . 35

Figure 4.2 Frequency-wavenumber domain after the 2D-FFT of FE results for Alu-Tape2 with Alu modes (GMM) as yellow dashed lines. . . 37

Figure 4.3 Slowness-frequency domain after the STFT of FE results for AluTape2 with Alu modes (GMM) as yellow dashed lines. . . . . . . 38

Figure $4.4 h_{t}$ variation for Tape printed for the A0, S0 and A1 modes. . . . 40

Figure 4.5 $h_{t}$ variation for Alu-Tape printed for the first three modes. . . . . 40

Figure 4.6 $E_{t}$ variation for Tape printed for the A0, S0 and A1 modes. . . . 42

Figure $4.7 \quad E_{t}$ variation for Alu-Tape printed for the first three modes. . . . 42

Figure $4.8 \quad \nu_{t}$ variation for Tape printed for the A0 to A2 modes. . . . . . . 43

Figure $4.9 \quad \nu_{t}$ variation for Alu-Tape printed for the first few modes. . . . . . 43

Figure 4.10 Waveguide FE results for a 75 element double layered Alu-Tape plate. . . . . . . . . . . . . . . . 45

Figure 5.1 Relation of the sensitive points between the different representations. 48

Figure 5.2 Influence of a variation of one parameter at a time on the minima (o), maxima (x) and points of maximum slope (•). . . . . . . 50 
Figure 6.1 Structure of the forward and inverse problem. . . . . . . . . . 51

Figure 6.2 Result of the global matrix method for Alu-Tape and Alu. . . . . 52

Figure 6.3 Slowness-minima for the first three Alu-Tape modes closest to the A0 Alu mode. ................... 53

Figure 6.4 Two parameter optimization path for $f_{\mathrm{s} 12}$ and $f_{\mathrm{sl} 3}$ of FEM results. 58

Figure 6.5 Three parameter optimization path for $f_{\mathrm{sl} 2}, f_{\mathrm{sl} 3}$ and $f_{\mathrm{cr} 2}$ of FEM results. . . . . . . . . . . . . . . 59

Figure 6.6 Frequency-wavenumber domain after the 2D-FFT of FE results for Alu-Tape1 with Alu modes (GMM) as yellow dashed lines. . . 62

Figure 6.7 Slowness-frequency domain after the STFT of FE results for AluTape1 with Alu modes (GMM) as yellow dashed lines. . . . . . .

Figure 6.8 Frequency-wavenumber domain after the 2D-FFT of FE results for a triple layered plate (Tape2 in the middle) with Alu modes $($ GMM) as yellow dashed lines. . . . . . . . . . . . . . .

Figure 6.9 Slowness-frequency domain after the STFT of FE results for a triple layered plate (Tape2 in the middle) with Alu modes (GMM) as yellow dashed lines. . . . . . . . . . . . . .

Figure A.1 $h_{t}$ variation for in-plane FEM results of Alu-Tape with Alu modes $(\mathrm{GMM})$ as yellow dashed lines. . . . . . . . . . . . .

Figure A.2 $h_{t}$ variation for out-of-plane FEM results of Alu-Tape with Alu modes (GMM) as yellow dashed lines. . . . . . . . . . . . .

Figure A.3 $E_{t}$ variation for in-plane FEM results of Alu-Tape with Alu modes (GMM) as yellow dashed lines. . . . . . . . . . . . 70

Figure A.4 $E_{t}$ variation for out-of-plane FEM results of Alu-Tape with Alu modes (GMM) as yellow dashed lines. . . . . . . . . . . . 71

Figure A.5 $\nu_{t}$ variation for in-plane FEM results of Alu-Tape with Alu modes (GMM) as yellow dashed lines. . . . . . . . . . . . . . 72

Figure A.6 $\quad \nu_{t}$ variation for out-of-plane FEM results of Alu-Tape with Alu modes (GMM) as yellow dashed lines. . . . . . . . . . . . 


\section{SUMMARY}

This research focuses on the application of guided waves techniques to nondestructively characterize the structural integrity of bonded engineering components. Computational methods are used to examine the properties of multi-layered, adhesive bonded plates. This study quantifies the effect of the adhesive bond parameters (Young's modulus, Poisson's ration and bond thickness) on the dispersion curves. A commercial finite element (FE) code (ABAQUS/Explicit) is used for the numerical model while the global matrix method and the waveguide FE method are used to benchmark the resulting dispersion relationships in the form of a frequencywavenumber or slowness-frequency relation. The postprocessing of FE data includes the two-dimensional Fourier transform (2D-FFT) and the short-time Fourier transform (STFT). Note that the 2D-FFT and STFT operate on multiple or just one transient output signals of the FE results respectively, while the waveguide FE method uses mass-, damping- and stiffness-matrices to generate the dispersion relations. In the dispersion relations, a set of bond parameter sensitive and FE-visible points is selected. The frequency locations of these points represent the solution criteria for the inversion procedure based on the global matrix method. The capabilities of the inversion process depend on the number of transient output signals from an FE simulation for the forward problem. 


\section{CHAPTER I}

\section{INTRODUCTION}

Improved structural integrities combined with low element weight widen the range of possible applications for adhesive bonded components. This and advantages like low cost and ease of assembly compared to conventional bonding techniques have heightened the demand for adhesive bonded engineering structures. Adhesive bonded components are being increasingly used in many lightweight engineering fields such as the airplane industry. Aging, thermal and mechanical influences are only a few mechanisms that could lead to failure or damage. Failure of any component in this field could induce critical situations for humans which is definitely the most unwanted consequence of any possible incident. As a result, maintenance and reliability are crucial subjects. Usually the adhesive bond itself is not accessible or visible. This makes nondestructive testing methods that are able to quantify the properties of hidden layers attractive.

The objective of this work is to monitor the quality of an hidden adhesive layer. This will be done by quantifying its properties using the dispersion relationships. The properties of the bond (in this case the Young's modulus, the Poisson's ratio and the bond thickness) will be centered around the values for a commercially available, double coated, adhesive transfer tape. This tape has a very high strength and is intended to replace rivets, screws and other mechanical fastenings. All three param-

eters influence the dispersion relation of guided waves. The whole structure itself would therefore be a waveguide consisting of layered media. Further on, properties of the outer adherent are assumed to be known, which also means that the dispersion relation of the top layer by itself is also known. 
In this work computational methods are focused on a double layered plate first, and then possibilities for more than two layers and eventual experiments are identified. Different methods to solve the forward problem, in other words methods to produce the dispersion relation, are compared to each other, while a finite element simulation is used to model the idealized reality. Between the forward and the inverse problem, different sets of parameter sensitive points as an input for the inverse procedure are examined and discussed. A set of sensitive points is selected by observing tendencies and used to solve the inverse problem via the global matrix method, while possibilities (including periodic non plate structures) for the waveguide FE method are investigated.

Previous research focused on the application of the finite element simulation of wave propagation in a waveguide [14, 20,21]. Signal processing methods including the two dimensional Fourier transformation and short-time Fourier transformation are investigated in [3] and [15] respectively. [6, 20] used numerical methods like the global matrix method to interpret experimental results of [6] as well as understand wave propagation in multi-layered plates. The waveguide FE method as one of the newer approaches is investigated in $[10,11]$. 


\section{CHAPTER II}

\section{THEORETICAL BACKGROUND OF WAVE PROPAGATION}

This chapter provides a brief introduction to wave propagation in elastic media. It is not intended to give a complete coverage of the subject, but only to provide a background to understand the topics covered in this work. For further reference and a more detailed description of the material, the reader is encouraged to see $[2,5,18]$.

Depending on material properties, geometry and method of excitation, different kinds of wave phenomena can be observed. First a short derivation of wave propagation theory is presented and then different wave types in infinite media and waveguides are covered.

\subsection{Governing equations in linear elasticity}

The media employed in this research are considered to be continuous, homogeneous, isotropic and linear elastic. The constitutive stress-strain relationship (Hooke's law) for the stress tensor $\tau_{i j}$ under these conditions is

$$
\tau_{i j}=\lambda \varepsilon_{k k} \delta_{i j}+2 \mu \varepsilon_{i j}
$$

where $\epsilon_{i j}$ is the strain tensor, related to the displacement $u_{i}$ by

$$
\varepsilon_{i j}=\frac{1}{2}\left(u_{i, j}+u_{j, i}\right)
$$


and $\mu$ and $\lambda$ are the Lamé constants. A relationship to Young's modulus $E$ and Poisson's ratio $\nu$ is given by

$$
\begin{aligned}
\lambda & =\frac{E \nu}{(1+\nu)(1-2 \nu)}, \\
\mu & =\frac{E}{2(1+\nu)} .
\end{aligned}
$$

The stress equation of motion is

$$
\tau_{i j, j}+\rho f_{i}=\rho \ddot{u}_{i}
$$

In this case body forces are neglected

$$
\rho f_{i}=0
$$

which leads to

$$
\tau_{i j, j}=\rho \ddot{u}_{i}
$$

If the strain-displacement relation (2.2) is substituted into Hooke's law (2.1) and the expression for the stress in terms of the displacements is subsequently substituted in the stress equation of motion (2.6), the displacement equation of motion

$$
\mu u_{i, j j}+(\lambda+\mu) u_{j, j i}=\rho \ddot{u}_{i}
$$

is obtained, which reads in vector representation as

$$
\mu \nabla^{2} \mathbf{u}+(\lambda+\mu) \nabla \nabla \cdot \mathbf{u}=\rho \ddot{\mathbf{u}}
$$

Navier's equations of motion (2.7) is a partial differential equation which cannot be solved for the displacements explicitly. Thus a solution for the displacements is assumed. If the solution is a sum of potentials the Helmholtz decomposition may be applied which provides a simpler set of equations for $\mathbf{u}$. The expression for $\mathbf{u}$ is

$$
\mathbf{u}=\nabla \varphi+\nabla \times \boldsymbol{\psi}
$$


Equation (2.8) represents the three components of the displacement $\mathbf{u}$ with the four functions $\varphi, \psi_{1}, \psi_{2}$ and $\psi_{3}$. To guarantee the uniqueness of the solution, an additional constraint

$$
\nabla \cdot \psi=0
$$

is introduced. Substitution of (2.8) into the displacement equation of motion (2.7) leads to two uncoupled wave equations expressed in terms of the displacement potentials $\varphi$ and $\boldsymbol{\psi}$ :

$$
c_{\mathrm{L}}^{2} \nabla^{2} \varphi=\frac{\partial^{2}}{\partial t^{2}} \varphi, \quad c_{\mathrm{T}}^{2} \nabla^{2} \boldsymbol{\psi}=\frac{\partial^{2}}{\partial t^{2}} \boldsymbol{\psi}
$$

where $c_{\mathrm{L}}$ represents the wave speed of longitudinal waves and $c_{\mathrm{T}}$ the wave speed of transverse shear waves,

$$
c_{\mathrm{L}}^{2}=\frac{\lambda+2 \mu}{\rho}, \quad c_{\mathrm{T}}^{2}=\frac{\mu}{\rho} .
$$

Two basic types of waves are associated with each of the potentials in equation (2.10). Longitudinal waves correspond to the potential $\varphi$ and shear waves are associated with the potential $\boldsymbol{\psi}$. These waves are called body waves. Figure 2.1 shows the particle motion of these two basic types of waves.

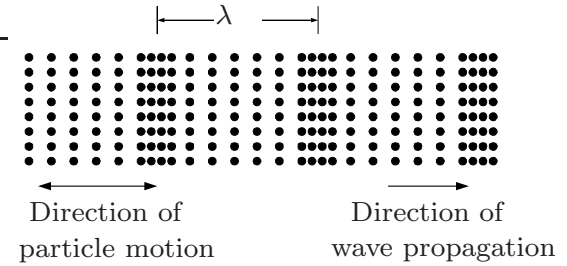

(a) Longitudinal wave

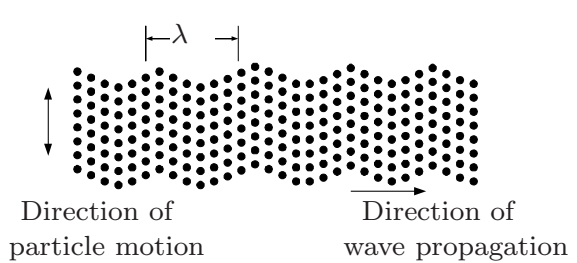

(b) Shear wave

Figure 2.1: Particle motion of body waves

\subsection{Wave phenomena}

Plane waves are assumed to have constant properties $(\epsilon, \tau, u)$ on a plane perpendicular to its direction of propagation $\mathbf{p}$

$$
\mathbf{u}=\mathbf{d} f(\mathbf{x} \cdot \mathbf{p}-c t)
$$


where $\mathbf{d}$ is the unit vector defining the direction of particle motion, and $c$ is either the longitudinal wave speed $c_{\mathrm{L}}$ or the transverse wave speed $c_{\mathrm{T}}$. By substituting (2.12) into (2.7b), one obtains

$$
\left(\mu-\rho c^{2}\right) \mathbf{d}+(\lambda+\mu)(\mathbf{p} \cdot \mathbf{d}) \mathbf{p}=0 .
$$

Since $\mathbf{p}$ and $\mathbf{d}$ are two different unit vectors, it can be seen that the two possible solutions that form the basis of wave propagation are either $\mathbf{d}= \pm \mathbf{p}$ or $\mathbf{p} \cdot \mathbf{d}=0$ :

1) $\mathbf{d}= \pm \mathbf{p}$ leads to $\mathbf{p} \cdot \mathbf{d}= \pm 1$. Inspection of (2.13) yields $c=c_{\mathrm{L}}$ as defined in (2.11). Since $\mathbf{d}$ and $\mathbf{p}$ are linearly dependent, this represents a particle movement in the direction of propagation - a longitudinal or P-wave.

2) $\mathbf{p} \cdot \mathbf{d}=0$ yields with (2.13), $c=c_{\mathrm{T}}$ (see (2.11)). Hence, the direction of motion is normal to the direction of propagation, and the wave is called a transverse wave. If a two-dimensional plane of propagation is considered (for example, the $\left(x_{1}, x_{2}\right)$-plane), a wave with an in-plane displacement (in the $\left(x_{1}, x_{2}\right)$-plane) is called an SV-wave (vertically polarized), while a wave with out-of-plane displacement (in the $x_{3}$-direction) is called an $\mathrm{SH}$-wave (horizontally polarized).

In a homogeneous, isotropic material, transverse and longitudinal wave speeds are independent of frequency - therefore they are nondispersive.

\subsection{Reflection of body waves}

Here the most important types of reflections of body waves are presented. To understand the reflection phenomena, a halfspace with a stress free surface $\left(\tau_{22}=0\right.$ and $\left.\tau_{21}=0\right)$ is considered. For a wave propagating in an infinite media, plane-strain case, the displacement field of an harmonic wave in the $x_{1}, x_{2}$ plane is

$$
\mathbf{u}^{(w)}=A_{n} \mathbf{d}^{(w)} \mathrm{e}^{\imath k_{w}\left(x_{1} p_{1}^{(w)}+x_{2} p_{2}^{(w)}-c_{w} t\right)},
$$


where $(w)$ denotes the wave (longitudinal or transverse), $k_{n}=\frac{\omega}{c_{n}}$ is the wavenumber of the wave $n$ and the respective wave speeds are $c_{n}$. First the case of an incident P-wave (longitudinal wave) is explained.

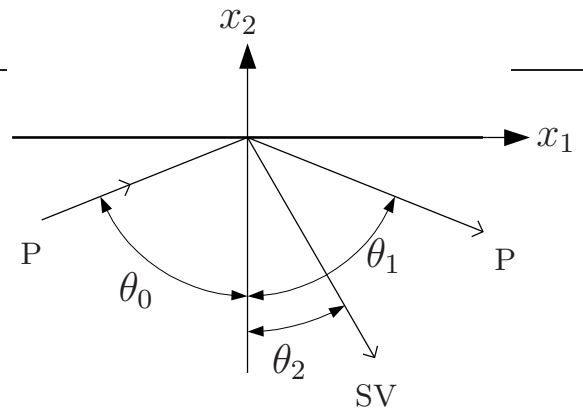

(a) incident P-wave

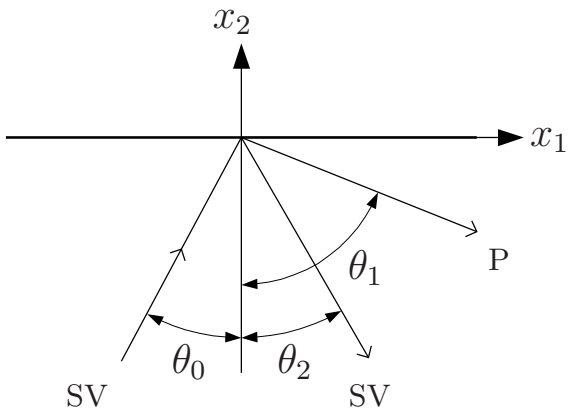

(b) incident SV-wave

Figure 2.2: Reflection of body waves.

Figure 2.2(a) shows that an incident P-wave produces two reflected waves, a Pwave and a SV-wave (shear wave). The boundary condition of a stress free surface requires that an incident $\mathrm{P}$-wave under an arbitrary angle is reflected as both, a $\mathrm{P}$ and SV-wave. The effect of a single incident wave-type producing two different waves after reflection from a boundary is called mode conversion. If the incident angle $\theta_{0}$ approaches zero, only a $\mathrm{P}$-wave is reflected. It may also be recognized that $\theta_{2}$ (the reflected angle of the $\mathrm{SV}$-wave) is always smaller than $\theta_{1}$. Hence for the range $0<\theta_{0}<\frac{\pi}{2}$ always two reflected waves exist with $\theta_{0}=\theta_{1}$. To obtain non-trivial amplitudes $A_{n}$, the angles of incident and reflected waves $\theta_{0}, \theta_{1}$ and $\theta_{2}$ as defined in Figure 2.2, must satisfy Snell's law:

$$
k_{0} \sin \theta_{0}=k_{1} \sin \theta_{1}=k_{2} \sin \theta_{2} .
$$

Now the reflection of vertically polarized shear waves is considered. Figure 2.2(b) shows that again two waves are reflected.

However in this case the reflecting angle of the $\mathrm{P}$-wave (i.e. $\theta_{1}$ ) is larger than $\theta_{2}$. 


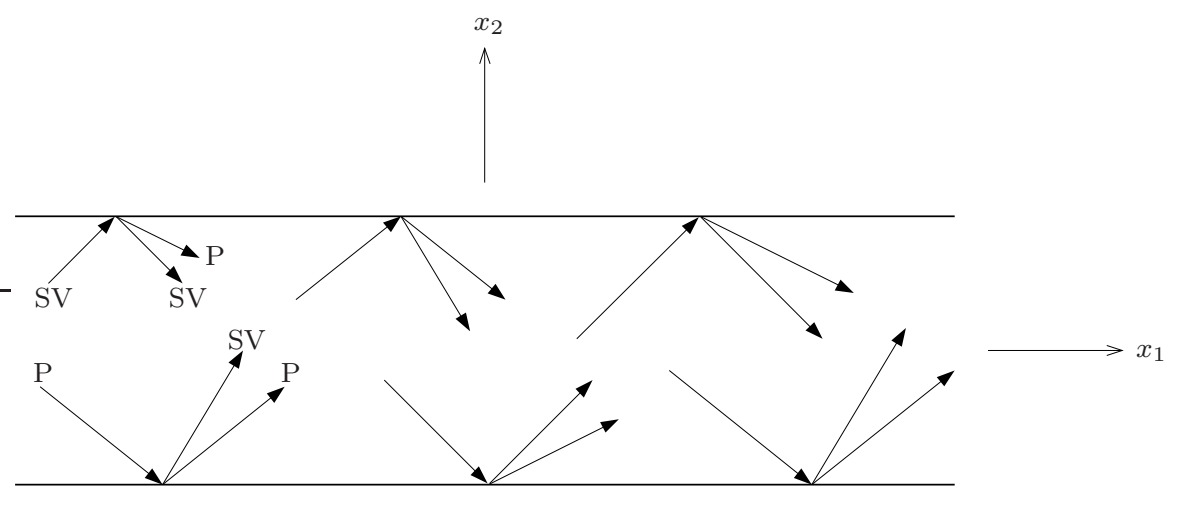

Figure 2.3: Reflections in a waveguide.

That means above a certain value of $\theta_{0}$ (this angle is called the critical angle)

$$
\theta_{\text {critical }}=\arcsin \frac{c_{\mathrm{T}}}{c_{\mathrm{L}}}
$$

the reflected P-wave vanishes and a so called evanescent surface wave is established. This wave travels along the surface and decays in $x_{2}$-direction. Below $\theta_{\text {critical }}$ the relation $\theta_{0}=\theta_{2}$ is valid. At an incident angle of $\theta_{0}=0^{\circ}$ there is only a reflected SV-wave. In [13] several other cases of reflections are discussed.

Other wave types like Rayleigh waves, Stoneley waves and Love waves are not discussed here since they are not relevant for this work. A detailed description of these waves can be found in $[2,5]$.

\subsection{Guided waves}

Guided waves occur in bodies with finite dimensions in two directions, like plates, shells, etc. These types of bodies are called waveguides. The waves travel in the direction of the infinite extensions of the body. Because of the boundaries of the body, the waves are reflected multiple times, as shown in Figure 2.3. These multiple reflections form a constructive/destructive interference pattern in the waveguide. This pattern can be interpreted as standing waves in the $x_{2}$-direction, while travelling in the $x_{1}$-direction. 
To investigate wave motion in an elastic wave guide, the potentials

$$
\varphi=\Phi\left(x_{2}\right) \exp \imath\left(k x_{1}-\omega t\right), \quad \psi_{3}=\Psi\left(x_{2}\right) \exp \imath\left(k x_{1}-\omega t\right)
$$

are used. The direction of propagation is in the $x_{1}$-direction. Assuming a plate thickness of $2 h$, plane strain and stress-free boundaries at $x_{2}= \pm h$ finally lead to the Rayleigh-Lamb frequency equations [2]

$$
\begin{aligned}
& \frac{\tan (q h)}{\tan (p h)}=-\frac{4 k^{2} p q}{\left(q^{2}-k^{2}\right)^{2}} \\
& \frac{\tan (q h)}{\tan (p h)}=-\frac{\left(q^{2}-k^{2}\right)^{2}}{4 k^{2} p q}
\end{aligned}
$$

where

$$
p^{2}=\frac{\omega^{2}}{c_{\mathrm{L}}^{2}}-k^{2}, \quad q^{2}=\frac{\omega^{2}}{c_{\mathrm{T}}^{2}}-k^{2} .
$$

Equation (2.18a) represents the symmetric Lamb modes, while (2.18b) characterizes the antisymmetric Lamb modes. A symmetric mode is defined to have a displacement symmetric to the $x_{1}$-axis, and an antisymmetric mode is accordingly determined by an antisymmetric displacement with respect to the $x_{1}$-axis.

The waves in a plate are called Lamb waves. The waves propagating in a waveguide are determined by the wavenumber $k$ and the frequency $f$. For a given wavenumber waves with different frequencies can propagate in a waveguide. Possible frequencywavenumber combinations form modes. The solutions of the Rayleigh-Lamb frequency equation (2.18) form several branches in a frequency-wavenumber diagram. Each of these branches is one propagating mode.

The dispersion curves are obtained by finding first a numerical solution in the $(\omega, k)$-domain or $(f, k)$-domain and differentiating $f$ numerically (partially with respect to $k$ ) for each of the different modes to attain the group velocity in the absence of attenuation as

$$
c_{\mathrm{gr}}(f)=2 \pi \frac{\partial f}{\partial k}
$$


Group velocity as defined in Equation (2.20) describes the velocity of propagating energy. In contrast, points of constant phase propagate with the phase velocity $c_{\mathrm{ph}}=$ $\frac{\omega}{k}$. Note that Lamb waves are dispersive, therefore the propagation velocity of a Lamb mode is dependent on its oscillation frequency. However, for nondispersive media, group and phase velocity are equal.

$$
s l(f)=\frac{1}{c_{\mathrm{gr}}(f)}
$$

(2.21) is used to express the dispersion relationship of Lamb modes in terms of the energy slowness $s l$, which is the reciprocal of the group velocity. Figures 2.4 and 2.5 show the dispersion curves of the Rayleigh-Lamb spectrum for a $1 \mathrm{~mm}$ thick aluminum plate.

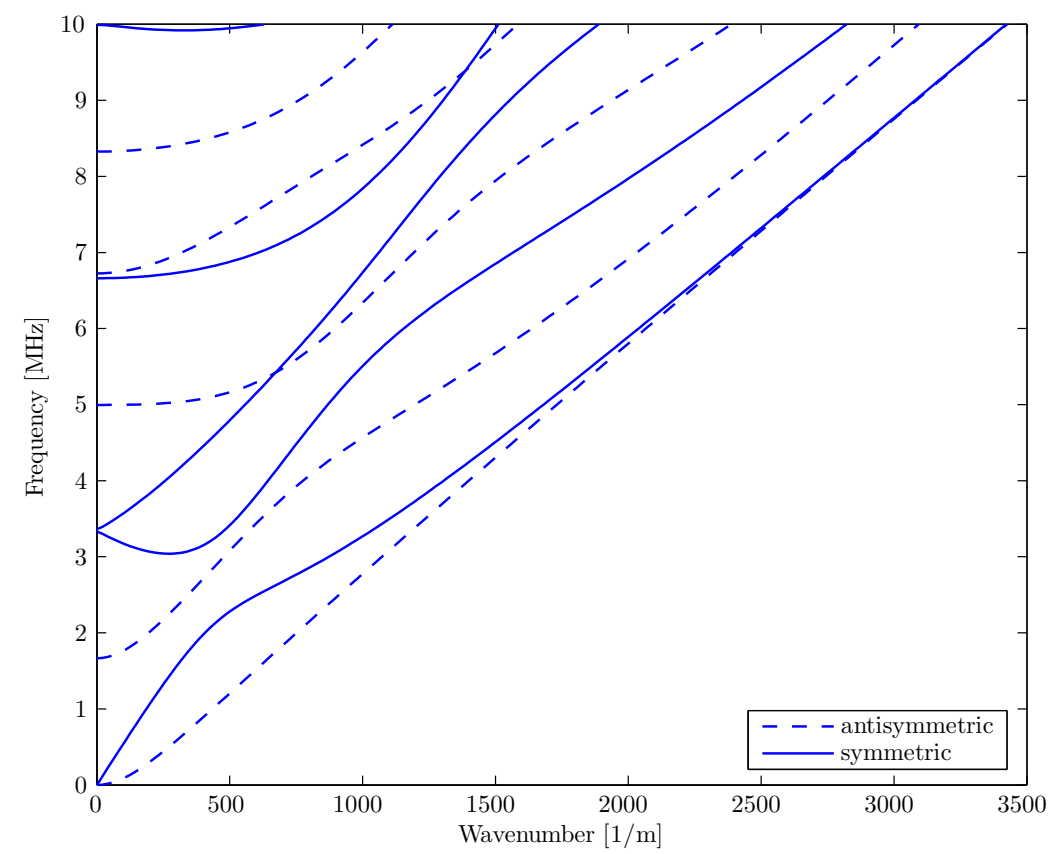

Figure 2.4: Dispersion relation in the frequency-wavenumber domain. 


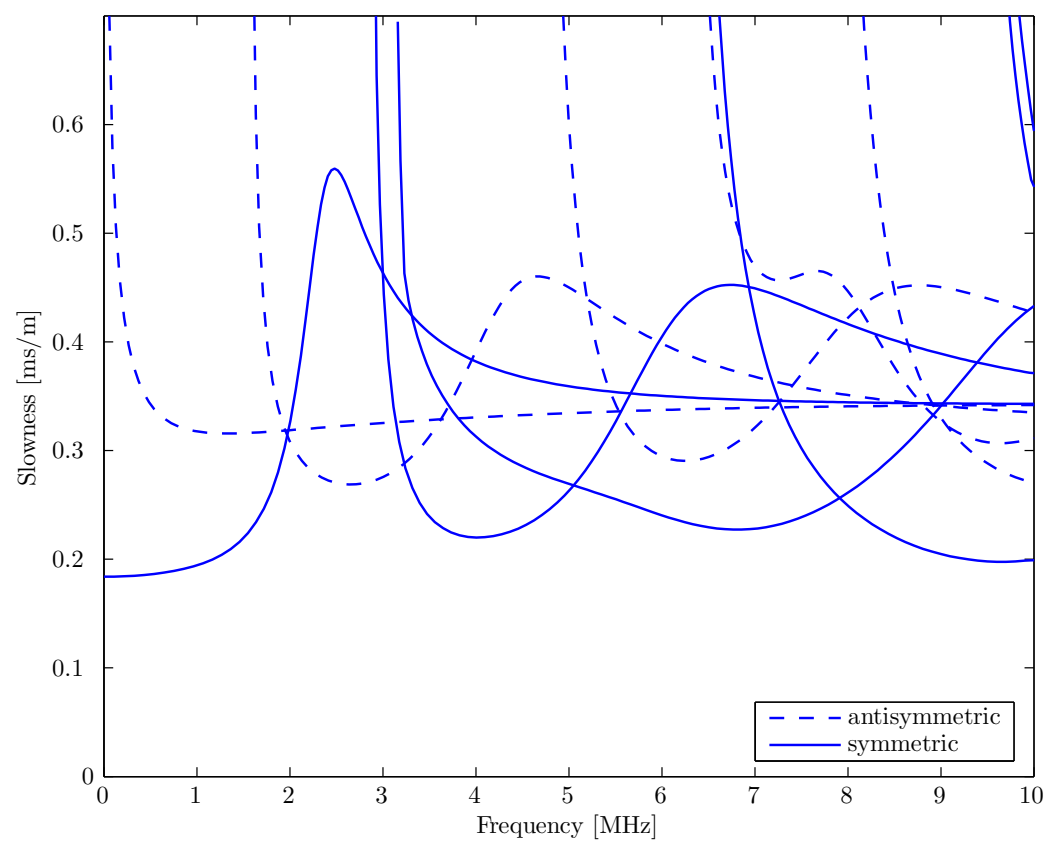

Figure 2.5: Dispersion relation in the slowness-frequency domain. 


\section{CHAPTER III}

\section{METHODS FOR LAYERED MEDIA}

In this research different methods to examine the dispersion curves of multi-layered plates are used. This section explains the theoretical background of those methods as well as the procedures themselves.

\subsection{Global matrix method (GMM)}

The global matrix method is a well known method to calculate the dispersion curves of multilayered media. The Rayleigh-Lamb wave equation is only valid for a waveguide consisting out of one (linearly elastic) material. In order to solve for the dispersion relation of multiple layers in this section the global matrix method is used. The method has already proven to perform well, because it is used in a software for dispersion curves called Disperse [17]. This work does not present any new information considering the GMM, therefore only the basics of this method are described.

Figure 3.1 shows the geometrical shape and the material properties of the double layer model examined in this research. The top and bottom layer are assumed to be stress-free on their surfaces. The interface conditions between layers are continuity of the displacement and traction vector. This means that the two layers are rigidly joined to each other at the interface. A detailed description of the model for layered

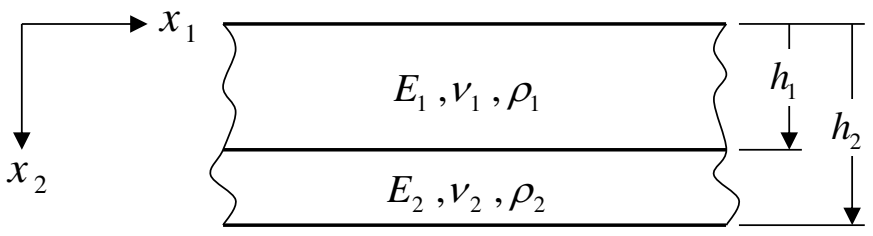

Figure 3.1: Double layer model for the GMM. 
plates is done by [18].

\subsubsection{Equation of motion - Displacement}

Assuming elastic and isotropic materials for both layers it is obvious that the displacement field $\mathbf{u}^{(s)}=\left[u_{1}^{(s)}, u_{2}^{(s)}, u_{3}^{(s)}\right]^{T}$ in each layer must satisfy Navier's displacement equations of motion

$$
\mu^{(s)} \nabla^{2} \mathbf{u}^{(s)}+\left(\lambda^{(s)}+\mu^{(s)}\right) \nabla\left(\nabla \cdot \mathbf{u}^{(s)}\right)=\rho^{(s)} \ddot{\mathbf{u}}^{(s)} .
$$

The different layers are denoted by the index $s \in(1,2)$. For displacement fields that vary harmonically with $\mathbf{u}^{(s)}=\mathbf{u}^{(s)} \mathrm{e}^{\imath \omega t}$ this leads to

$$
\mu^{(s)} \nabla^{2} \mathbf{u}^{(s)}+\left(\lambda^{(s)}+\mu^{(s)}\right) \nabla\left(\nabla \cdot \mathbf{u}^{(\mathbf{s})}\right)=\rho^{(s)} \omega^{2} \mathbf{u}^{(\mathbf{s})} .
$$

The Helmholz decomposition is used to determine the displacement field in each layer

$$
\mathbf{u}^{(s)}=\nabla \phi^{(s)}+\nabla \times \boldsymbol{\psi}^{(s)} .
$$

Again a plane-strain case is assumed, which means that the displacement is only a function of $x_{1}$ and $x_{2}$ and that $u_{3}^{(s)} \equiv 0$. By using the Helmholtz decomposition this can be achieved when $\psi_{1}^{(s)}=\psi_{2}^{(s)} \equiv 0, \psi_{3}^{(s)}=\psi_{3}^{(s)}\left(x_{1}, x_{2}, t\right)$ and $\phi^{(s)}=\phi^{(s)}\left(x_{1}, x_{2}, t\right)$. With these restrictions on $\phi^{(s)}$ and $\boldsymbol{\psi}^{(s)}$, equation (3.3) yields the displacement components

$$
\begin{aligned}
u_{1}^{(s)} & =\frac{\partial}{\partial x_{1}} \phi^{(s)}\left(x_{1}, x_{2}, t\right)-\frac{\partial}{\partial x_{2}} \psi_{3}^{(s)}\left(x_{1}, x_{2}, t\right), \\
u_{2}^{(s)} & =\frac{\partial}{\partial x_{2}} \phi^{(s)}\left(x_{1}, x_{2}, t\right)+\frac{\partial}{\partial x_{1}} \psi_{3}^{(s)}\left(x_{1}, x_{2}, t\right), \\
u_{3}^{(s)} & \equiv 0 .
\end{aligned}
$$

If (3.3) is substituted into (3.1), the result is two uncoupled wave equations

$$
\begin{gathered}
\frac{\partial^{2}}{\partial x_{2}^{2}} \phi^{(s)}\left(x_{1}, x_{2}, t\right)+\frac{\partial^{2}}{\partial x_{1}^{2}} \phi^{(s)}\left(x_{1}, x_{2}, t\right)-\frac{\ddot{\phi}^{(s)}\left(x_{1}, x_{2}, t\right)}{\left(c_{L}^{(s)}\right)^{2}}=0, \\
\frac{\partial^{2}}{\partial x_{2}^{2}} \psi_{3}^{(s)}\left(x_{1}, x_{2}, t\right)+\frac{\partial^{2}}{\partial x_{1}^{2}} \psi_{3}^{(s)}\left(x_{1}, x_{2}, t\right)-\frac{\ddot{\psi}_{3}^{(s)}\left(x_{1}, x_{2}, t\right)}{\left(c_{T}^{(s)}\right)^{2}}=0,
\end{gathered}
$$


where $c_{L}^{(s)}$ and $c_{T}^{(s)}$ represent the longitudinal and transversal wave speeds in layer $n$. Harmonic solutions to (3.8) in each layer can be written as

$$
\begin{aligned}
& \phi^{(s)}=C_{1}^{(s)} \mathrm{e}^{\imath\left(k_{L}\left(x_{1} \sin \theta_{L}^{(s)}+x_{2} \cos \theta_{L}^{(s)}\right)-\omega t\right)}+C_{2}^{(s)} \mathrm{e}^{\imath\left(k_{L}\left(x_{1} \sin \theta_{L}^{(s)}-x_{2} \cos \theta_{L}^{(s)}\right)-\omega t\right)}, \\
& \psi_{3}^{(s)}=C_{3}^{(s)} \mathrm{e}^{\imath\left(k_{T}\left(x_{1} \sin \theta_{T}^{(s)}+x_{2} \cos \theta_{T}^{(s)}\right)-\omega t\right)}+C_{4}^{(s)} \mathrm{e}^{\imath\left(k_{T}\left(x_{1} \sin \theta_{T}^{(s)}-x_{2} \cos \theta_{T}^{(s)}\right)-\omega t\right)},
\end{aligned}
$$

where

$$
k_{L}^{(s)}=\frac{\omega}{c_{L}^{(s)}}, \quad k_{T}^{(s)}=\frac{\omega}{c_{T}^{(s)}},
$$

and $C_{1}^{(s)}, C_{2}^{(s)}, C_{3}^{(s)}, C_{4}^{(s)}$ are arbitrary constants. The relation between the wavenumber $k$, the longitudinal wavenumber $k_{L}^{(s)}$ and the transverse wavenumber $k_{T}^{(s)}$ in each layer as well as the orthogonal wavenumbers $K_{L}^{(s)}$ and $K_{T}^{(s)}$ can be expressed as

$$
\begin{aligned}
K_{L}^{(s)} & =\sqrt{k_{L}^{2}-k^{2}}, \\
K_{T}^{(s)} & =\sqrt{k_{T}^{2}-k^{2}} .
\end{aligned}
$$

In order to replace the angles $\theta_{L}^{(s)}$ and $\theta_{T}^{(s)}$ in (3.9) and (3.10) the trigonometric relations

$$
\begin{aligned}
k & =k_{L}^{(s)} \sin \theta_{L}^{(s)}, \\
k & =k_{T}^{(s)} \sin \theta_{T}^{(s)}, \\
K_{L}^{(s)} & =k_{L}^{(s)} \cos \theta_{L}^{(s)}, \\
K_{T}^{(s)} & =k_{T}^{(s)} \cos \theta_{T}^{(s)},
\end{aligned}
$$

are used, this leads to

$$
\begin{aligned}
& \phi^{(s)}\left(x_{1}, x_{2}, t\right)=C_{1}^{(s)} \mathrm{e}^{\imath\left(x_{1} k+x_{2} K_{L}^{(s)}-\omega t\right)}+C_{2}^{(s)} \mathrm{e}^{\imath\left(x_{1} k-x_{2} K_{L}^{(s)}-\omega t\right)}, \\
& \psi_{3}^{(s)}\left(x_{1}, x_{2}, t\right)=C_{3}^{(s)} \mathrm{e}^{\imath\left(x_{1} k+x_{2} K_{T}^{(s)}-\omega t\right)}+C_{4}^{(s)} \mathrm{e}^{\imath\left(x_{1} k-x_{2} K_{T}^{(s)}-\omega t\right)} .
\end{aligned}
$$


Further on plugging the last equation into (3.4) and (3.5) results in

$$
\begin{aligned}
u_{1}^{(s)}=\imath & {\left[k\left(C_{1}^{(s)} \mathrm{e}^{\imath\left(x_{1} k+x_{2} K_{L}^{(s)}-\omega t\right)}+C_{2}^{(s)} \mathrm{e}^{\imath\left(x_{1} k-x_{2} K_{L}^{(s)}-\omega t\right)}\right)\right.} \\
& \left.-K_{T}^{(s)}\left(C_{3}^{(s)} \mathrm{e}^{\imath\left(x_{1} k+x_{2} K_{T}^{(s)}-\omega t\right)}-C_{4}^{(s)} \mathrm{e}^{\imath\left(x_{1} k-x_{2} K_{T}^{(s)}-\omega t\right)}\right)\right], \\
u_{2}^{(s)}=\imath & {\left[K_{L}^{(s)}\left(C_{1}^{(s)} \mathrm{e}^{\imath\left(x_{1} k+x_{2} K_{L}^{(s)}-\omega t\right)}-C_{2}^{(s)} \mathrm{e}^{\imath\left(x_{1} k-x_{2} K_{L}^{(s)}-\omega t\right)}\right)\right.} \\
+ & \left.k\left(C_{3}^{(s)} \mathrm{e}^{\imath\left(x_{1} k+x_{2} K_{T}^{(s)}-\omega t\right)}+C_{4}^{(s)} \mathrm{e}^{\imath\left(x_{1} k-x_{2} K_{T}^{(s)}-\omega t\right)}\right)\right] .
\end{aligned}
$$

\subsubsection{Strain and Stress}

The strain field in each layer is found from the displacement field by using the relation

of (2.2). In the plane strain case the non zero components of the strain field in each layer are

$$
\begin{aligned}
\varepsilon_{11}^{(s)} & =\frac{\partial u_{1}^{(s)}}{\partial x_{1}}, \\
\varepsilon_{22}^{(s)} & =\frac{\partial u_{2}^{(s)}}{\partial x_{2}}, \\
\varepsilon_{12}^{(s)} & =\frac{1}{2}\left(\frac{\partial u_{1}^{(s)}}{\partial x_{2}}+\frac{\partial u_{2}^{(s)}}{\partial x_{1}}\right) .
\end{aligned}
$$

With the Equation (3.20) and (3.21) for the displacement field the strain components in $x_{1^{-}}$and $x_{2}$-direction are

$$
\begin{aligned}
\varepsilon_{11}^{(s)}= & -k^{2}\left(C_{1}^{(s)} \mathrm{e}^{\imath\left(k x_{1}+x_{2} K_{L}^{(s)}-\omega t\right)}+C_{2}^{(s)} \mathrm{e}^{\imath\left(k x_{1}-x_{2} K_{L}^{(s)}-\omega t\right)}\right) \\
& +k K_{T}^{(s)}\left(C_{3}^{(s)} \mathrm{e}^{\imath\left(k x_{1}+x_{2} K_{T}^{(s)}-\omega t\right)}-C_{4}^{(s)} \mathrm{e}^{\imath\left(x_{1} k-x_{2} K_{T}^{(s)}-\omega t\right)}\right), \\
\varepsilon_{22}^{(s)}= & +K_{L}^{(s) 2}\left(C_{1}^{(s)} \mathrm{e}^{\imath\left(x_{1} k+x_{2} K_{L}^{(s)}-\omega t\right)}+C_{2}^{(s)} \mathrm{e}^{\imath\left(x_{1} k-x_{2} K_{L}^{(s)}-\omega t\right)}\right) \\
& +k K_{T}^{(s)}\left(C_{3}^{(s)} \mathrm{e}^{\imath\left(x_{1} k+x_{2} K_{T}^{(s)}-\omega t\right)}-C_{4}^{(s)} \mathrm{e}^{\imath\left(x_{1} k-x_{2} K_{T}^{(s)}-\omega t\right)}\right),
\end{aligned}
$$

and the shear deformation is

$$
\begin{aligned}
\varepsilon_{12}^{(s)}= & +k K_{L}^{(s)}\left(C_{1}^{(s)} \mathrm{e}^{\imath\left(x_{1} k+x_{2} K_{L}^{(s)}-\omega t\right)}-C_{2}^{(s)} \mathrm{e}^{\imath\left(x_{1} k-x_{2} K_{L}^{(s)}-\omega t\right)}\right) \\
& +\left(k^{2}-K_{T}^{(s) 2}\right)\left(C_{3}^{(s)} \mathrm{e}^{\imath\left(x_{1} k+x_{2} K_{T}^{(s)}-\omega t\right)}+C_{4}^{(s)} \mathrm{e}^{\imath\left(x_{1} k-x_{2} K_{T}^{(s)}-\omega t\right)}\right) .
\end{aligned}
$$

The stress field is calculated from the strain with Hooke's law. Using (2.1) and the fact that only $\tau_{22}^{(s)}$ and $\tau_{12}^{(s)}$ are relevant for the boundary conditions of each layer 
yields

$$
\begin{aligned}
\tau_{22}^{(s)}= & \left(\lambda^{(s)} k_{L}^{(s) 2}+2 \mu^{(s)} K_{L}^{(s) 2}\right)\left(C_{1}^{(s)} \mathrm{e}^{\imath\left(k x_{1}+K_{L}^{(s)} x_{2}-\omega t\right)}+C_{2}^{(s)} \mathrm{e}^{\imath\left(k x_{1}-K_{L}^{(s)} x_{2}-\omega t\right)}\right) \\
& +2 \mu^{(s)} k K_{T}^{(s)}\left(C_{3}^{(s)} \mathrm{e}^{\imath\left(k x_{1}+K_{T}^{(s)} x_{2}-\omega t\right)}-C_{4}^{(s)} \mathrm{e}^{\imath\left(k x_{1}-K_{T}^{(s)} x_{2}-\omega t\right)}\right)
\end{aligned}
$$

and

$$
\begin{aligned}
\tau_{12}^{(s)}= & 2 \mu^{(s)} k K_{L}^{(s)}\left(C_{1}^{(s)} \mathrm{e}^{\imath\left(k x_{1}+K_{L}^{(s)} x_{2}-\omega t\right)}-C_{2}^{(s)} \mathrm{e}^{\imath\left(k x_{1}-K_{L}^{(s)} x_{2}-\omega t\right)}\right) \\
& +\mu^{(s)}\left(K_{T}^{(s) 2}-k^{2}\right)\left(C_{3}^{(s)} \mathrm{e}^{\imath\left(k x_{1}+K_{T}^{(s)} x_{2}-\omega t\right)}+C_{4}^{(s)} \mathrm{e}^{\imath\left(k x_{1}-K_{T}^{(s)} x_{2}-\omega t\right)}\right) .
\end{aligned}
$$

\subsubsection{Boundary conditions}

At each of the upper and lower stress free surfaces the stresses $\tau_{22}$ and $\tau_{12}$ are zero. This leads to two equations for each free surface. At an interface between two layers the stresses $\tau_{22}$ and $\tau_{12}$ and the displacement $u_{1}$ and $u_{3}$ must be equal in both layers. This yields four equations for each interface. A stress free upper surface at $x_{2}=0$ for layer 1 leads to

$$
\tau_{22}^{(1)}=0, \quad \tau_{12}^{(1)}=0
$$

The continuity of displacement and stresses at the interface $x_{2}=h_{1}$ between layer 1 and layer 2 is expressed by

$$
\begin{array}{ll}
\tau_{22}^{(1)}=\tau_{22}^{(2)}, & \tau_{12}^{(1)}=\tau_{12}^{(2)}, \\
u_{1}^{(1)}=u_{1}^{(2)}, & u_{2}^{(1)}=u_{2}^{(2)} .
\end{array}
$$

The stress free lower surface at $x_{2}=h_{2}$ for layer 2 is described by

$$
\tau_{22}^{(2)}=0, \quad \tau_{12}^{(2)}=0
$$

Using (3.20) and (3.21) for the displacements and (3.28) and (3.29) for the stresses in Equations (3.30) to (3.33) leads to 8 equations which can be represented in a 
$8 \times 8$ matrix

$$
\underbrace{\left[\begin{array}{cccc}
A_{1,1} & A_{1,2} & \ldots & A_{1,8} \\
A_{2,1} & A_{2,2} & \ldots & A_{2,8} \\
\vdots & \vdots & \ddots & \vdots \\
A_{8,1} & A_{8,2} & \ldots & A_{8,8}
\end{array}\right]} \underbrace{\left[\begin{array}{c}
C_{1}^{(1)} \\
C_{2}^{(1)} \\
\vdots \\
C_{4}^{(2)}
\end{array}\right]}=\left[\begin{array}{c}
0 \\
0 \\
\vdots \\
0
\end{array}\right] .
$$

A

C

All elements of the matrix $\mathbf{A}$ are functions of the known material properties $E^{(s)}, \nu^{(s)}, \rho^{(s)}$, the unknown angular frequency $\omega$ and wavenumber $k$. In order to get the dispersion curves, the eigenvalue problem

$$
\operatorname{det}(\mathbf{A})=0
$$

has to be solved. The eigenvalues of Equation (3.35) are the frequency-wavenumber relationship of the different modes. This special kind of eigenvalue problem has to be solved numerically.

Plates with more than two layers have more interface conditions which leads to the general expression that a $s$-layer model needs $4 \cdot s$ equations from the boundary conditions. Note also that $h_{2}$ in this case denotes the overall thickness. Below $h_{a}$ always refers to the thickness of the aluminum, while $h_{t}$ refers to the thickness of the adhesive bond (tape). In all double layer cases the aluminum is on top of the bond which leads to $h_{1}=h_{a}$ and $h_{2}=h_{a}+h_{t}$. 


\subsection{Finite element method (FEM)}

The finite element method has a variety of applications and is still evolving. This work uses the commercial software Abaqus/Explicit. Here the theoretical background of the FEM is presented briefly, whereas the details of the used FE models are described. For further reference the author recommends [1] and [23].

\subsubsection{FE theory}

The general equation of motion in matrix form for the dynamic finite element simulation in terms of nodal displacements $\mathbf{u}_{\mathrm{e}}$ is given as

$$
\mathbf{M} \ddot{\mathbf{u}}_{\mathrm{e}}+\mathbf{C} \dot{\mathbf{u}}_{\mathrm{e}}+\mathbf{K} \mathbf{u}_{\mathrm{e}}-\mathbf{f}=\mathbf{0}
$$

where $\mathbf{f}$ is the vector of applied forces and

$$
\begin{aligned}
\mathbf{M} & =\int_{V} \rho \mathbf{N}^{T} \mathbf{N} \mathrm{d} V, \\
\mathbf{C} & =\int_{V} c \mathbf{N}^{T} \mathbf{N} \mathrm{d} V, \\
\mathbf{K} & =\int_{V}(\mathfrak{D N})^{T} \mathfrak{E} \mathfrak{D N} \mathrm{d} V,
\end{aligned}
$$

are mass matrix, damping matrix and the stiffness matrix respectively. Those relations are based on

$$
\begin{aligned}
& \mathbf{u}=\mathbf{N u}_{\mathrm{e}}, \\
& \boldsymbol{\tau}=\mathfrak{E} \varepsilon,
\end{aligned}
$$

where the mass density is denoted by $\rho$, the mechanical stiffness matrix is given by $\mathfrak{E}$, whereas $\mathbf{N}$ are interpolation functions and $\mathfrak{D}$ denotes differential operators to realize strains from the nodal displacements $\varepsilon=(\mathfrak{D N}) \mathbf{u}_{\mathrm{e}}$. 


\subsubsection{FE program}

Both packages of Abaqus can be used to solve dynamic problems in the form of Equation (3.36) for the displacement vector $\mathbf{u}_{\mathrm{e}}$. Abaqus/Standard solves the problem via an implicit algorithm, which means that the solution for dynamic quantities at the time $t+\Delta t$ is not only based on the values at $t$, but also on the same quantities at $t+\Delta t$. The result are nonlinear equations which make the whole procedure numerically stable but slow. The Abaqus/Explicit algorithm is computationally efficient for the analysis of large models with relatively short dynamic response times, which makes it optimal for high frequency simulations. Explicit schemes, as used in ABAQUS/Explicit, obtain values for dynamic quantities at $t+\Delta t$ based entirely on available values at time $t$.

\subsubsection{FE criteria}

In this section two fundamental finite element criteria are described, which influence the accuracy and costs of a simulation.

\subsubsection{Integration time step}

The explicit procedure integrates through time by using many small time increments. The central difference operator is only conditionally stable, the stability limit being approximately equal to the time for an elastic wave to cross the smallest element dimension in the model. The default setting of Abaqus/Explicit is to calculate this time step on its own

$$
\triangle t \approx \frac{l_{e \min }}{c_{L}},
$$

where $l_{e \min }$ is the smallest element dimension in the mesh and $c_{L}$ is the wave speed of longitudinal waves defined by equation (2.11). The estimate for $\Delta t$ is only approximate, therefore the conservative (safe) time estimate for 2D models is

$$
\triangle t=\frac{l_{e \min }}{\sqrt{2} \cdot c_{L}} .
$$


The automatic time step calculation of Abaqus/Explicit produces good results. However the software also allows the user to choose the time step. But two facts have to been taken into consideration. If the time step is too long, high frequency vibrations are not resolved accurately and the solution process might become unstable while if the time step is too short a lot of calculation time is wasted. A usually good rule [14] for the relation of the time increment $\Delta t$ and the maximal frequency of interest $f_{\max }$ is given by

$$
\triangle t=\frac{1}{20 f_{\max }}
$$

\subsubsection{Element size}

The size of the elements must be chosen so that the propagating waves are accurately captured. In [3] it is recommended that more than 10 nodes per wavelength are used, while [14] and [20] use a higher number. This can be expressed by

$$
l_{e}=\frac{1}{20 k_{\max }}
$$

with

$$
k_{\max }=\frac{1}{\lambda_{\min }}
$$

where $l_{e}$ is the element length, $\lambda_{\min }$ is the shortest wavelength and $k_{\max }$ is the maximum wavenumber of interest. The shortest wavelength can be approximated by the transverse wave speed $c_{T}$ and the maximum frequency that should be simulated. This leads to the overall relation

$$
l_{e}=\frac{\lambda_{\min }}{20} \approx \frac{c_{T}}{20 f_{\max }}
$$

\subsubsection{Nyquist criteria}

In addition to the FE criteria concerning the integration time step and the element length, the Nyquist criteria is important. For the 2D-FFT that is performed on the 
data to be accurate, the sampling frequency in time and space have to be adjusted, because the 2D FFT entails sampling a continuous signal with a given sampling rate. If the continuous signal contains frequencies above half the sampling frequency the frequency content for the sampled data is falsified. This effect is called aliasing and further reference can be found in [16]. The Nyquist frequency is the upper frequency limit for the continuous signal in order to avoid aliasing. This means for practical purposes that the continuous signal has to be sampled at least twice within the period of the highest frequency

$$
\triangle T=\frac{1}{2 f_{\max }}
$$

Hence the necessary output time step $\triangle T$ is $5 \cdot 10^{-8}$ seconds in order to avoid aliasing up to a maximum frequency of $10 \mathrm{Mhz}$. A similar relationship

$$
\triangle X=\frac{1}{2 \kappa_{\max }}
$$

is valid for the sampling in space with $\triangle X$ as the length between two output points.

\subsubsection{FE model}

Just like the previous sections stated, different parameters of the FE model are crucial for the success of a simulation. Two FE models are set up in Abaqus/Explicit to model multimodal wave propagation in a double layer plate.

The FE models use 2D solid structural elements (CPE4R) consisting of four nodes with two degrees of freedom (DOFs) at each node. The DOFs of the plane strain elements are the translation in $x_{1}$ and $x_{2}$ direction. As an absorbing boundary a set of elements called infinite elements (CINPE4) is used. These elements represent one layer at each end of the plate and are supposed to simulate an infinite plate without any reflections from boundaries in $x_{1}$ direction. The infinite elements don't absorb $100 \%$ of the energy. Therefore minor reflections are the result. But those reflections don't influence the main results. 
Figure 3.2 shows a high resolution model that satisfies all requirements concerning the Nyquist and FE criteria and offers a high enough resolution of the results for the inversion procedure. Figure 3.3 shows the low resolution model that still satisfies all criteria but is restricted to the relative small frequency-wavenumber window of interest. This model offers a short computation time (1 hour compared to 24 hours for the high resolution model) and is used in chapter 4.3 for the parametric studies. The calculation end time $t_{\text {end }}=0.26 \mathrm{~ms}$ as well as the sampling frequency of $20 \mathrm{MHz}$ and the excitation are the same for both models.

Besides the computation time, Table 3.1 shows the other differences between the FE models. The mesh size is the total number of used elements, the DOFs are the total number of degrees of freedom while the memory in Megabyte quantifies the memory usage (RAM) during the computation. The preprocessing of such a model usually uses temporally more memory. The computer used for these calculations was a regular Pentium PC with a 3.2 GHz Dual-Core CPU and 2 GB of RAM. Although the presented models don't completely use the resources of this computer, models with slightly smaller element sizes, more than two layers or viscoelastic behavior will reach the limit.

The element length $l_{e}$ is the most critical parameter concerning computational costs. Both models try to keep the element shape as close as possible to a square in the aluminium layer, while $l_{e}$ in the out-of-plane direction in the bond layer is only half as long as in the aluminium layer. This is done to account for the slower wave speeds in the adhesive bond layer. For wave propagation purposes a uniform mesh is generally the best choice. Too big changes of element sizes or shapes result in multiple micro reflections that disturb the constructive/destructive interference pattern that is responsible for the properties of guided waves. Without presenting details here the presented models in Figures 3.2 and 3.3 show the best results of a variety of different simulations. 
In order to neglect near field effects in the results, the distance between the excitation and the first output signal has to be $10 \cdot h_{a}=10 \mathrm{~mm}$. Although all simulations record the in and out-of-plane velocity of the output nodes, for simplicity Figures 3.2 and 3.3 show the output signals as green arrows in out-of-plane direction only. The excitation in the same figures is pictured as a red downward arrow, which means, that only one node experiences an out-of-plane force. The force follows a triangular amplitude variation with an end time of 0.1 us to get a broad band excitation at a frequency range of up to $10 \mathrm{MHz}$.

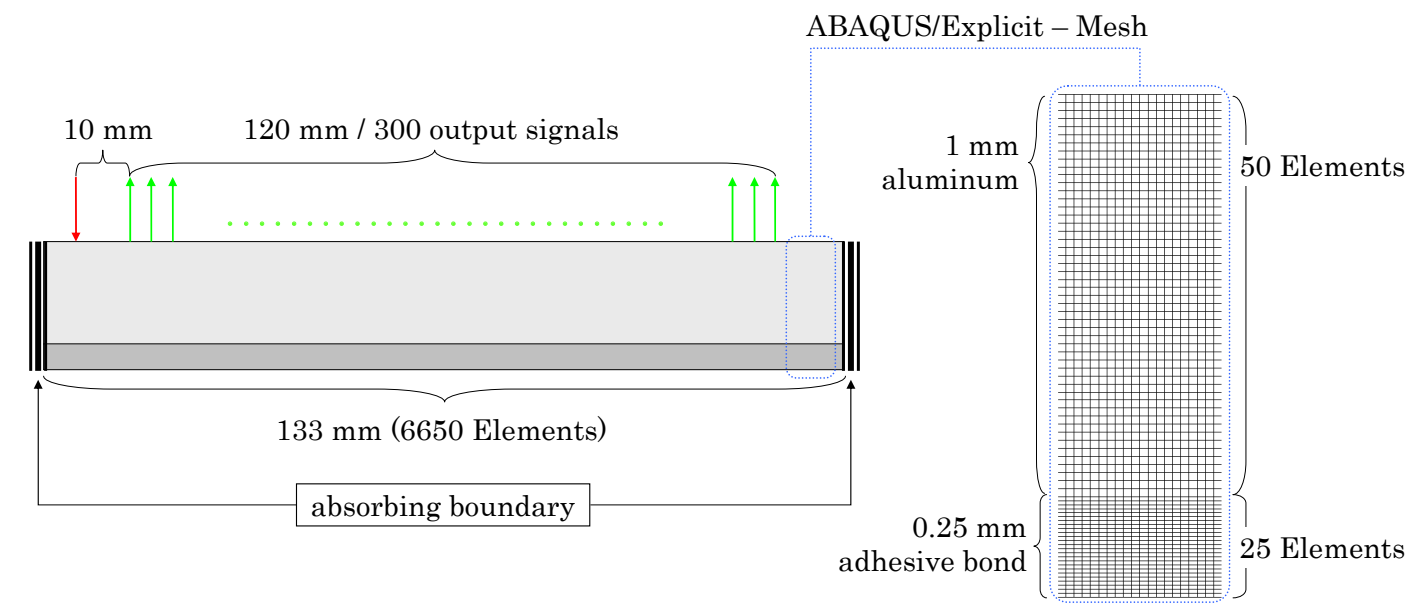

Figure 3.2: High resolution FE model.

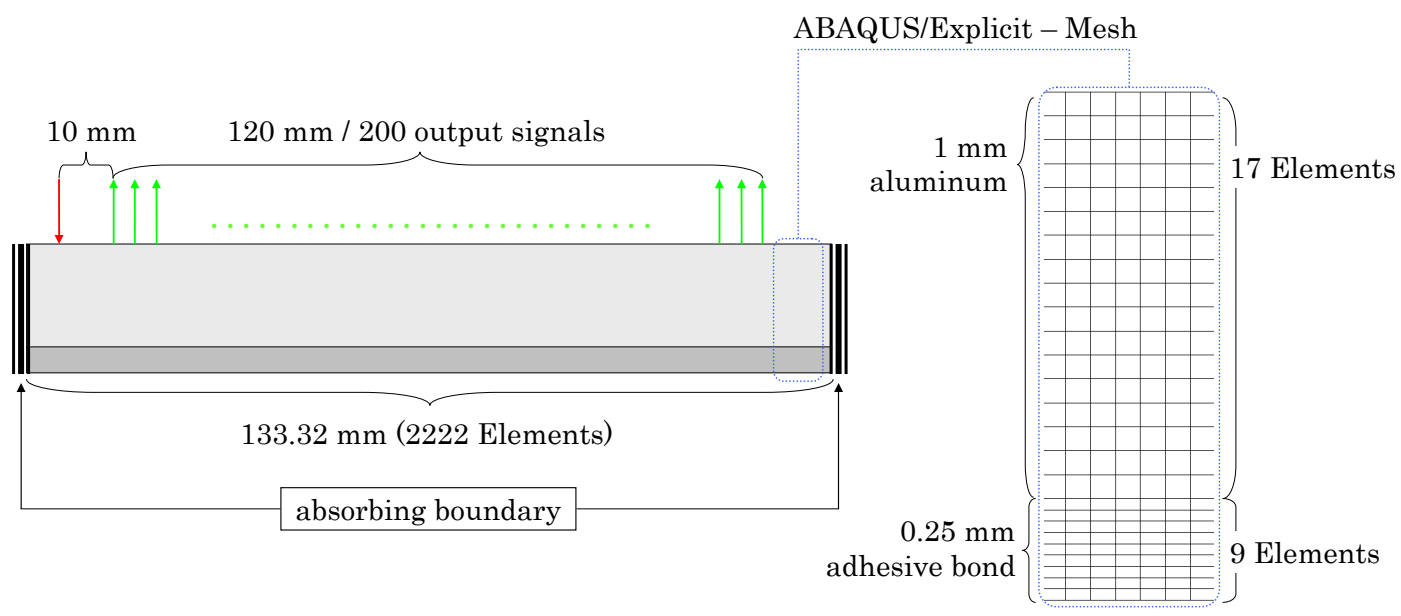

Figure 3.3: Low resolution FE model. 
Table 3.1: Low and high resolution FE model settings (rounded values).

\begin{tabular}{|l|c|c|c|c|c|}
\hline Model & Mesh size & DOF's & Memory & $l_{e}[\mathrm{~mm}]$ & $\triangle X[\mathrm{~mm}]$ \\
\hline Low resolution & 60,000 & 120,000 & $50 \mathrm{MB}$ & 0.06 & 0.6 \\
\hline High resolution & 500,000 & $1,000,000$ & $400 \mathrm{MB}$ & 0.02 & 0.4 \\
\hline
\end{tabular}

\subsubsection{Signal processing}

The results of the Finite Element simulation are represented as transient output signals of the in-plane and out-of-plane velocities.

Most experimentalists use only one transient output signal for simplicity. The short-time Fourier transform transforms one output signal into its time-frequency representation. The use of the corresponding propagation distance leads to a group velocity-frequency or a slowness-frequency representation.

Another possibility is to use multiple output signals to generate the two dimensional Fourier transform, or a frequency - wavenumber representation. The following section explains those signal processing tools.

\subsubsection{Short-time Fourier transform (STFT)}

The short-time Fourier transform is based on a regular Fourier transform. In this method the signal is split up into a series of overlapping parts instead of transforming the entire transient signal at once. Each part is then windowed and Fourier transformed. The STFT is given by

$$
S_{\mathrm{stft}}\left(\omega_{0}, t_{0}\right)=\frac{1}{2 \pi} \int_{-\infty}^{+\infty} s(t) h\left(t-t_{0}\right) e^{-\imath \omega_{0} t} \mathrm{~d} t
$$

where $h(t)$ is a window function. The spectrogram of an STFT is defined as

$$
E_{\mathrm{d}}\left(\omega_{0}, t_{0}\right)=\left|S_{\mathrm{stft}}\left(\omega_{0}, t_{0}\right)\right|^{2}
$$


This corresponds to the energy density spectrum. The STFT needs only a single signal $s(t)$. So only one measurement at a single location is sufficient to determine the entire time-frequency representation. As a result a relation for the time $t$ and the circular frequency $\omega$ can be obtained which is converted to a slowness-frequency representation.

\subsubsection{Two dimensional Fourier transform (2D-FFT)}

In this section, a mathematical procedure is introduced in order to process and interpret numerically obtained data. The 2D-Fourier transform was originally used to analyze experimental results in the form of multimode time signals [3].

The 2D Fourier transform is essentially a Fourier transform of a Fourier transform. In the continuous space it can be calculated by

$$
S(k, \omega)=\int_{-\infty}^{+\infty} \int_{-\infty}^{+\infty} s(x, t) e^{-\imath(\omega t+k x)} \mathrm{d} x \mathrm{~d} t,
$$

where $s(x, t)$ can be any function of two variables. Usually $\mathrm{x}$ and $\mathrm{t}$ will correspond to two spatial directions or one spatial direction and time respectively. The two dimensional energy density spectrum is

$$
E_{\mathrm{d}}(\omega, k)=|S(\omega, k)|^{2} .
$$

in the continuous case. In order to apply the 2D transformation to numerically or experimentally obtained data, the discrete equivalent to (3.52) is used. The discrete form is called 2D-FFT (Fast Fourier Transformation). The data must be sampled spatially and temporally.

From a wave propagation point of view, the 2D Fourier transform maps the original time signal $s(x, t)$ to the signal $S(k, \omega)$ in the angular frequency-wavenumber domain. The advantage of this transformation is that different modes are easily distinguished, because every mode obeys its own distinct $(k, \omega)$-relation. 


\subsection{Waveguide FE method (WFE)}

This method serves as a basis to create dispersion relations in a different way compared to the earlier explained global matrix method. In order to apply this method ABAQUS/Standard is used to create one segment of a periodic structure. For our case this would mean one element layer in the in-plane direction. For further reference on this subject see $[10,11]$.

\subsubsection{Dynamic Stiffness Matrix}

The stiffness, damping and mass matrices respectively $\mathbf{K}, \mathbf{C}$ and $\mathbf{M}$ of the FE model are used to create the dynamic stiffness matrix $\mathbf{D}$ which accounts for time-harmonic system behavior in a waveguide

$$
\mathbf{D}(\omega)=\mathbf{K}+i \omega \mathbf{C}-\omega^{2} \mathbf{M}
$$

where

$$
\mathrm{Du}=\mathbf{f}
$$

In a general case the nodes in (3.54) are partitioned into sets of internal nodes (i),

nodes on the left side (l) and nodes on the right side (r), see Figure 3.4. If there are no forces on the internal nodes, this leads to

$$
\left[\begin{array}{ccc}
\mathrm{D}_{\mathrm{ii}} & \mathrm{D}_{\mathrm{il}} & \mathrm{D}_{\mathrm{ir}} \\
\mathrm{D}_{\mathrm{li}} & \mathrm{D}_{\mathrm{ll}} & \mathrm{D}_{\mathrm{lr}} \\
\mathrm{D}_{\mathrm{ri}} & \mathrm{D}_{\mathrm{rl}} & \mathrm{D}_{\mathrm{rr}}
\end{array}\right]\left[\begin{array}{c}
\mathbf{u}_{\mathrm{i}} \\
\mathbf{u}_{\mathrm{l}} \\
\mathbf{u}_{\mathrm{r}}
\end{array}\right]=\left[\begin{array}{c}
\mathbf{0} \\
\mathbf{f}_{\mathrm{l}} \\
\mathbf{f}_{\mathrm{r}}
\end{array}\right]
$$

Eliminating the interior degrees of freedom results in

$$
\left[\begin{array}{cc}
\tilde{\mathbf{D}}_{\mathrm{ll}} & \tilde{\mathbf{D}}_{\mathrm{lr}} \\
\tilde{\mathbf{D}}_{r l} & \tilde{\mathbf{D}}_{\mathrm{rr}}
\end{array}\right]\left[\begin{array}{l}
\mathbf{u}_{\mathrm{l}} \\
\mathbf{u}_{\mathrm{r}}
\end{array}\right]=\left[\begin{array}{l}
\mathbf{f}_{\mathrm{l}} \\
\mathbf{f}_{\mathrm{r}}
\end{array}\right],
$$




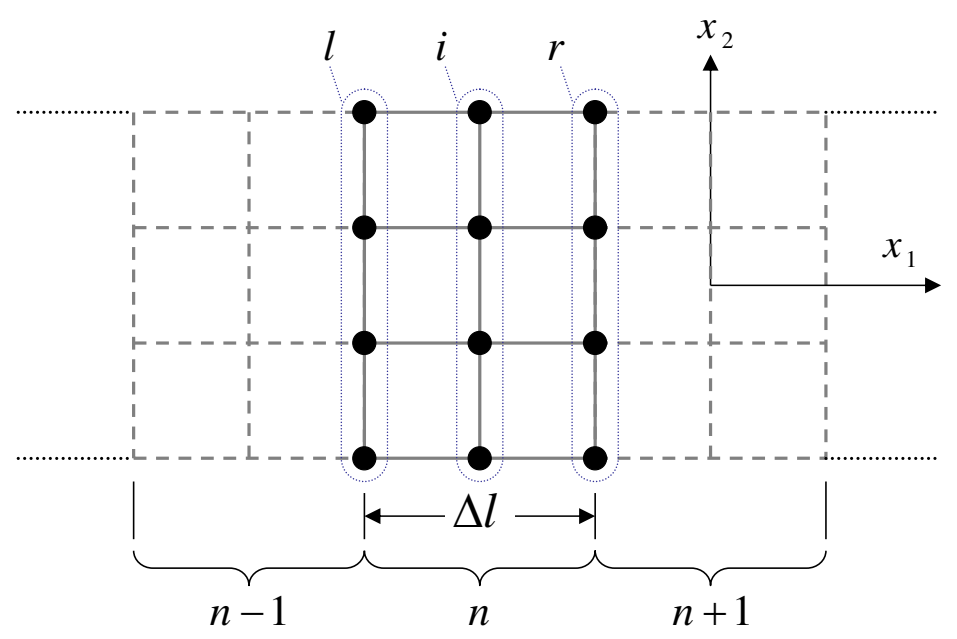

Figure 3.4: FE mesh of three sections in the waveguide.

with

$$
\begin{aligned}
& \tilde{\mathbf{D}}_{\mathrm{ll}}=\mathbf{D}_{\mathrm{ll}}-\mathbf{D}_{\mathrm{li}} \mathbf{D}_{\mathrm{ii}}^{-1} \mathbf{D}_{\mathrm{il}}, \\
& \tilde{\mathbf{D}}_{\mathrm{lr}}=\mathbf{D}_{\mathrm{lr}}-\mathbf{D}_{\mathrm{li}} \mathbf{D}_{\mathrm{ii}}^{-1} \mathbf{D}_{\mathrm{ir}}, \\
& \tilde{\mathbf{D}}_{\mathrm{rl}}=\mathbf{D}_{\mathrm{rl}}-\mathbf{D}_{\mathrm{ri}} \mathbf{D}_{\mathrm{ii}}^{-1} \mathbf{D}_{\mathrm{il}}, \\
& \tilde{\mathbf{D}}_{\mathrm{rr}}=\mathbf{D}_{\mathrm{rr}}-\mathbf{D}_{\mathrm{ri}} \mathbf{D}_{\mathrm{ii}}^{-1} \mathbf{D}_{\mathrm{ir}} .
\end{aligned}
$$

\subsubsection{Transfer matrix}

Since the entire periodic structure is made of single segments, the displacements and forces on the right side of one segment (n) have to be the same as those on the left side of the next segment $(n+1)$, what can be expressed by

$$
\begin{aligned}
& \mathbf{u}_{\mathrm{r}}^{(n)}=\mathbf{u}_{1}^{(n+1)} \\
& \mathbf{f}_{\mathrm{r}}^{(n)}=\mathbf{f}_{\mathrm{l}}^{(n+1)}
\end{aligned}
$$

Rearrangements lead to the frequency-dependent transfer matrix system representing a relation between the node variables of the bordering segments $(n)$ and $(n+1)$

$$
\left[\begin{array}{l}
\mathbf{u}_{1}^{(n+1)} \\
\mathbf{f}_{1}^{(n+1)}
\end{array}\right]=\mathbf{T}(\omega)\left[\begin{array}{c}
\mathbf{u}_{1}^{(n)} \\
\mathbf{f}_{1}^{(n)}
\end{array}\right]
$$


with

$$
\mathbf{T}(\omega)=\left[\begin{array}{cc}
-\tilde{\mathbf{D}}_{\mathrm{lr}}^{-1} \tilde{\mathbf{D}}_{\mathrm{ll}} & \tilde{\mathbf{D}}_{\mathrm{lr}}^{-1} \\
-\tilde{\mathbf{D}}_{\mathrm{rl}}+\tilde{\mathbf{D}}_{\mathrm{rr}} \tilde{\mathbf{D}}_{\mathrm{lr}}^{-1} \tilde{\mathbf{D}}_{\mathrm{ll}} & -\tilde{\mathbf{D}}_{\mathrm{rr}} \tilde{\mathbf{D}}_{\mathrm{lr}}^{-1}
\end{array}\right]
$$

The assumption of harmonic wave propagation in $x_{1}$-direction along the segment length $\triangle l$ with the complex wavenumber $\kappa$

$$
\begin{aligned}
& \mathbf{u}_{r}=\mathrm{e}^{\kappa \Delta l} \mathbf{u}_{l}, \\
& \mathbf{f}_{r}=\mathrm{e}^{\kappa \Delta l} \mathbf{f}_{l},
\end{aligned}
$$

leads to the eigenvalue problem

$$
\mathbf{T}(\omega)\left[\begin{array}{c}
\hat{\mathbf{u}}_{1}^{(n)}(\omega) \\
\hat{\mathbf{f}}_{1}^{(n)}(\omega)
\end{array}\right]=\lambda\left[\begin{array}{c}
\hat{\mathbf{u}}_{1}^{(n)}(\omega) \\
\hat{\mathbf{f}}_{1}^{(n)}(\omega)
\end{array}\right]=\mathrm{e}^{\kappa \Delta l}\left[\begin{array}{c}
\hat{\mathbf{u}}_{1}^{(n)}(\omega) \\
\hat{\mathbf{f}}_{1}^{(n)}(\omega)
\end{array}\right],
$$

which has $2 m$ solutions, where $m$ is the number of nodal DOFs on one side. The physical complex wavenumber is now calculated from the eigenvalues

$$
\kappa=\frac{\ln (\lambda)}{\triangle l}
$$

\subsubsection{Dispersion relation}

For each frequency the number of numerically calculated solutions for $\lambda$ is equal to the dimension of $\mathbf{T}$. But only a few eigenvalues represent a physically meaningful solution. Therefore only waves with $|\lambda| \leq 1$ are taken into account, while waves with $|\lambda| \ll 1$ and $\mathfrak{R e}\{\lambda\} \leq 1$ are not considered [10].

So far a frequency-wavenumber relation is generated. With this function it is relatively easy to calculate the phase velocity

$$
c_{p h}=\frac{\omega}{\kappa} .
$$

Furthermore group velocities are also very important. (2.20) represents one way to calculate the group velocities. This approach however leads to relatively big mismatches, due to the scanning approach of the waveguide-FE method. Another way 
to calculate the group velocity is to use the relation between the time average power $P$ and the time average overall energy density $\bar{E}$

$$
c_{g r}=\frac{P}{\bar{E}}
$$

In the frequency domain the power flow becomes

$$
P=-\frac{1}{2} \mathfrak{R e}\left\{\mathbf{f}^{\mathrm{H}} \dot{\mathbf{u}}\right\}=\frac{\omega}{2} \mathfrak{I m}\left\{\mathbf{f}^{\mathrm{H}} \mathbf{u}\right\}
$$

where the superscript $\mathrm{H}$ denotes the Hermitian, or complex conjugate transpose. The

total energy density $\bar{E}$ is the sum of the kinetic and potential energy densities $\bar{E}_{\mathrm{k}}$ and $\bar{E}_{\mathrm{p}}$ respectively

$$
\bar{E}=\bar{E}_{\mathrm{k}}+\bar{E}_{\mathrm{p}}
$$

where

$$
\begin{aligned}
& \bar{E}_{\mathrm{k}}=\frac{1}{4 \triangle l} \mathfrak{R e}\left\{\dot{\mathbf{u}}^{\mathrm{H}} \mathbf{M} \dot{\mathbf{u}}\right\}=-\frac{\omega^{2}}{4 \triangle l} \mathfrak{R e}\left\{\mathbf{u}^{\mathrm{H}} \mathbf{M} \mathbf{u}\right\} \\
& \bar{E}_{\mathrm{p}}=\frac{1}{4 \triangle l} \mathfrak{R e}\left\{\mathbf{u}^{\mathrm{H}} \mathbf{K} \mathbf{u}\right\} .
\end{aligned}
$$

\subsubsection{Example}

In order to verify the operability and the accuracy window, the waveguide FE-method is applied to a single aluminum plate. Two models are set up. One has eight elements, while the other one consists of twenty elements. Both use only one element layer in the plate direction $\left(x_{1}\right)$, which means that no internal nodes exist in this case and that $\triangle l=l_{e}$. Damping is neglected by setting $\mathbf{C}=\mathbf{0}$. Figure 3.5 shows the eight and the 20 element models. In order to calculate the dispersion curves in an effective way a square is chosen as element shape. The thickness $t$ is $1 \mathrm{~mm}$ in both cases resulting in an element length $l_{e}$ of $0.125 \mathrm{~mm}$ for the eight element model and $0.05 \mathrm{~mm}$ for the twenty element model. Figure 3.6 shows the results of the eight element and the twenty element model compared to each other. Due to the fact, that this method is based on matrices from an FE code the element size plays an important role in regard 
of the accuracy. The windows of maximum accuracy according to equations (3.45) to (3.47) are also pictured in the same figure. The good accuracy for the 8 element model as well as for the 20 element model inside those windows confirms equations (3.45) and (3.47) for the single plate. Further on this means that the GMM and the WFE produce the same results inside the suggested accuracy window.
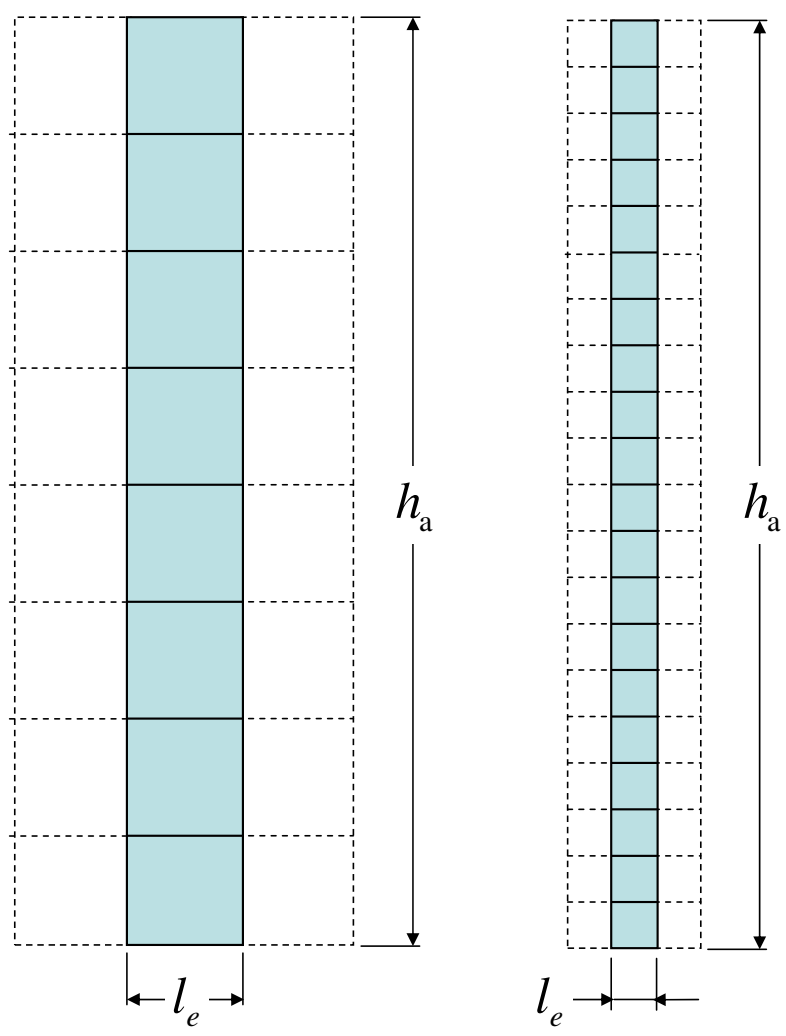

Figure 3.5: 8 element and 20 element models. 


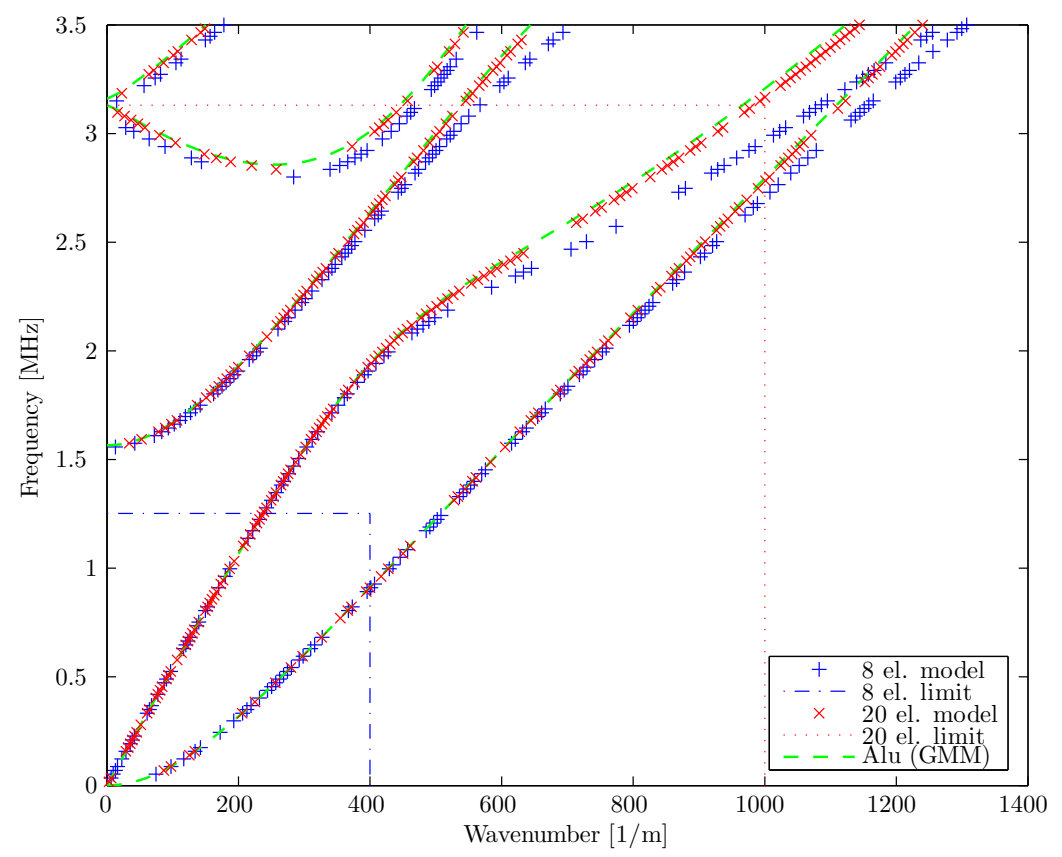

(a) Frequency-wavenumber relation

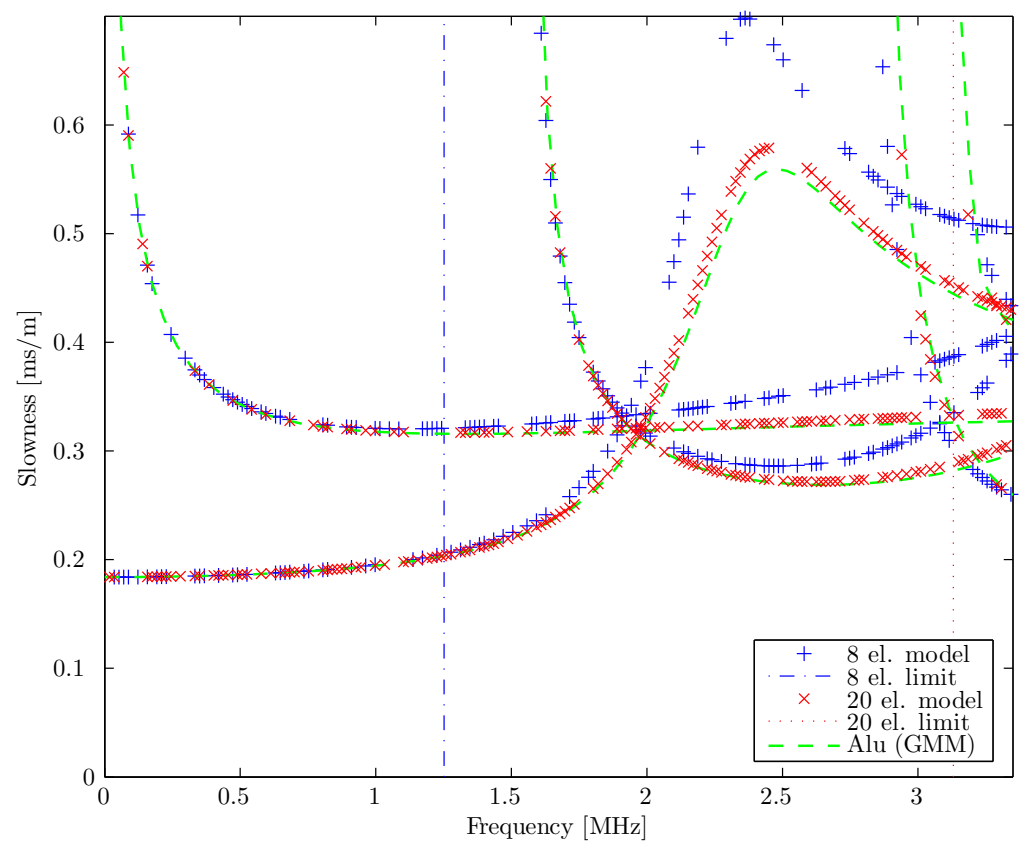

(b) Slowness-frequency relation

Figure 3.6: Waveguide FE results for a 8 element and a 20 element model of a 1 $\mathrm{mm}$ aluminum plate. 


\section{CHAPTER IV}

\section{FORWARD PROBLEM}

In order to investigate the influence of the tape parameters on the dispersion relation we want to use first the global matrix method to create the dispersion curves. In this process the program Disperse (Imperial College) [17] is used. These results are then compared to the results of the FEM simulation after some signal processing. Two representations are used, the frequency-wavenumber representation via the $2 \mathrm{D}$-FFT for multiple transient output signals and the slowness-frequency representation via the STFT for one transient output signal.

In order to stay comparable to former experiments and results in this field this research is centered around the adhesive bond properties that have been used in $[6,20]$. Table 4.1 shows the properties of the aluminium, tape 1 and tape 2 . In this chapter the focus is on the relative high stiffness tape 2. The lower stiffness tape 1 will be considered at a later time.

Table 4.1: Material properties.

\begin{tabular}{|l|c|c|c|}
\hline Material & Young's Modulus & Poisson's ration & Density \\
\hline Aluminium & $E_{a}=70.758 \mathrm{GPa}$ & $\nu_{a}=0.3375$ & $\rho_{a}=2700 \mathrm{~kg} / \mathrm{m}^{3}$ \\
\hline Tape 1 & $E_{t}=0.005 \mathrm{GPa}$ & $\nu_{t}=0.49$ & $\rho_{t}=1106 \mathrm{~kg} / \mathrm{m}^{3}$ \\
\hline Tape 2 & $E_{t}=1 \mathrm{GPa}$ & $\nu_{t}=0.35$ & $\rho_{t}=1106 \mathrm{~kg} / \mathrm{m}^{3}$ \\
\hline
\end{tabular}




\subsection{Dispersion relations for single and double lay- ered plates (GMM)}

Figure 4.1 shows the first three modes for a $0.25 \mathrm{~mm}$ single layer of adhesive bond (tape 2) called "Tape2", a 1 mm single layer of aluminum called "Alu" and a double layer of $1 \mathrm{~mm}$ aluminum and $0.25 \mathrm{~mm}$ adhesive bond (tape 2) called "Alu-Tape2". In both representations it becomes clear that the aluminum modes of a single plate have a strong influence on the modes of the double layer plate. The first Alu-Tape2 mode starts with the A0 Alu mode while the second Alu-Tape2 mode starts with the S0 Alu mode and crosses A0 Alu mode after that. The third Alu-Tape2 mode (cut-off frequency of $0.6 \mathrm{MHz}$ ) does not start with any Alu mode, but gets under the influence of the A0 Alu mode first and then crosses the S0 Alu mode. In the frequency-wavenumber representation it becomes clear that only one at a time of the combined modes follows an A0 Alu mode.

\subsection{Typical FE results}

Figure 4.2 shows the frequency-wavenumber domain of in-plane and out-of-plane FE results via the 2D-FFT. Figure 4.3 pictures the slowness-frequency domain of in-plane and out-of-plane FE results via the STFT.

In both representations the Alu-Tape2 modes are not visible everywhere. This is because it is assumed that only the top surface of the aluminum is accessible and the displacement profiles of the Alu-Tape2 modes change with frequency and wavenumber. Although the displacement profiles will not play a major role in this work we still want to make some statements here. In [20] those displacement profiles are examined in detail. A single combined Alu-Tape2 mode in the frequency-wavenumber representation can be divided into parts where it has a very low slope, parts where it has a higher slope and into parts where the slope changes (transition zones). During the low slope parts most of the modes have only significant in-plane displacements in 


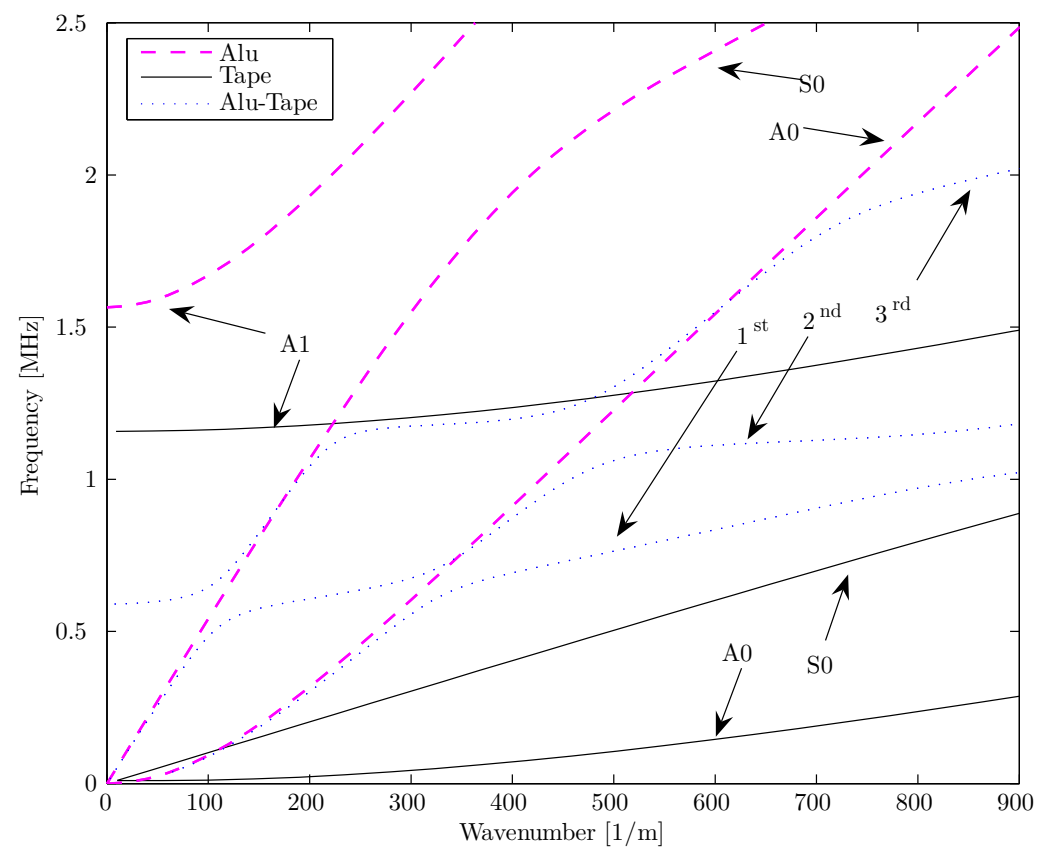

(a) Frequency-wavenumber relation

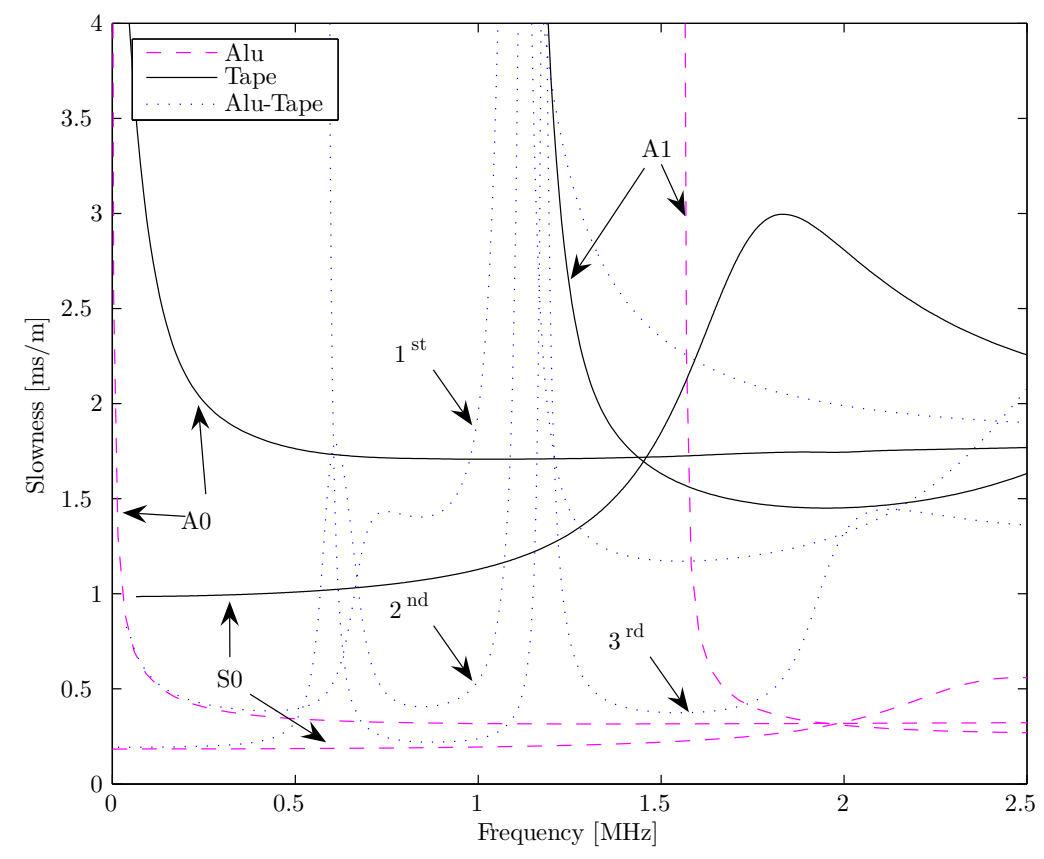

(b) Slowness-frequency relation

Figure 4.1: GMM for Alu, Tape2 and Alu-Tape2. 
the adhesive layer and almost no displacements in the aluminum layer [20]. During a high slope part, especially where the Alu-Tape2 mode crosses the Alu mode, the modes have significant in-plane and out-of-plane displacement in the tape and in the aluminum layer. There the displacement in the aluminum dominates the displacement in the tape layer. In other words the displacement field in the aluminum layer of an Alu-Tape mode and the displacement field of an Alu mode close to the Alu-Tape mode are similar. This explains why only the aluminum modes are measured for Alu-Tape [6]. The Alu-Tape2 modes show up only when they are close to the single plate aluminum modes and are therefore influenced by the single aluminum plate. Otherwise the visible dispersion relations of the FE results show the same behavior as predicted by the Global Matrix Method.

The difference of the in-plane and out-of-plane results are the same for both representations. The out-of-plane results show in general less information than the in-plane results. The second to fourth Alu-Tape2 modes under the influence of the S0 Alu mode are not or hardly visible. Further on the fifth and sixth Alu-Tape2 mode close to the A1 Alu mode bleach out at low frequencies.

Due to the fact that the infinite elements, mentioned in section 3.2.5, don't absorb $100 \%$ of the energy, a small reflection of the first Alu-Tape2 mode can be seen in the upper left corner of Figures 4.3(a) and 4.3(b). But those reflections do not influence any of the procedures in this work. 


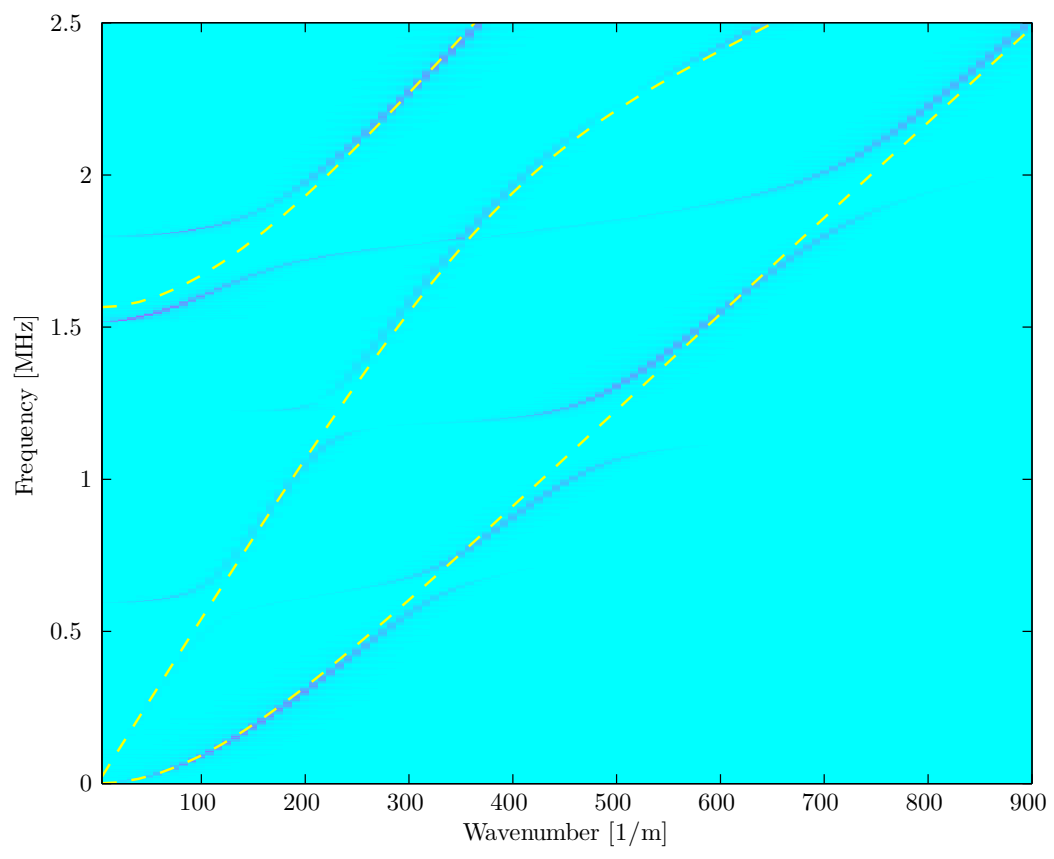

(a) In-plane component

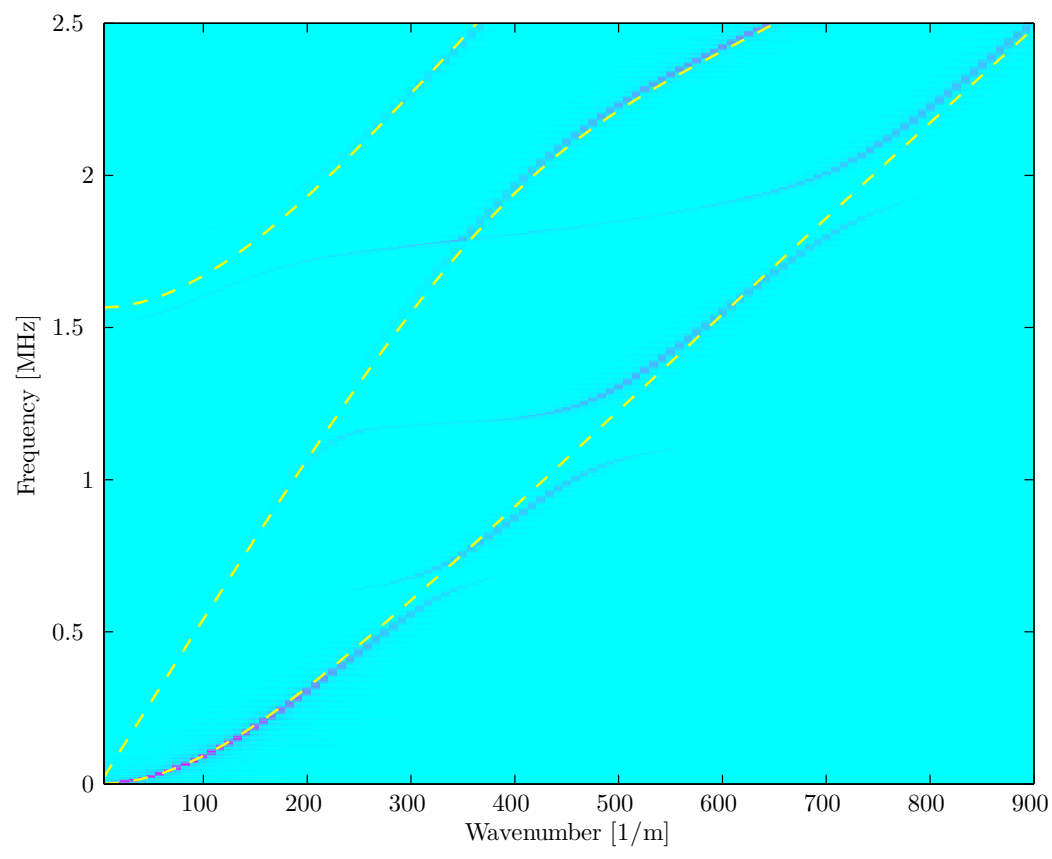

(b) Out-of-plane component

Figure 4.2: Frequency-wavenumber domain after the 2D-FFT of FE results for Alu-Tape2 with Alu modes (GMM) as yellow dashed lines. 


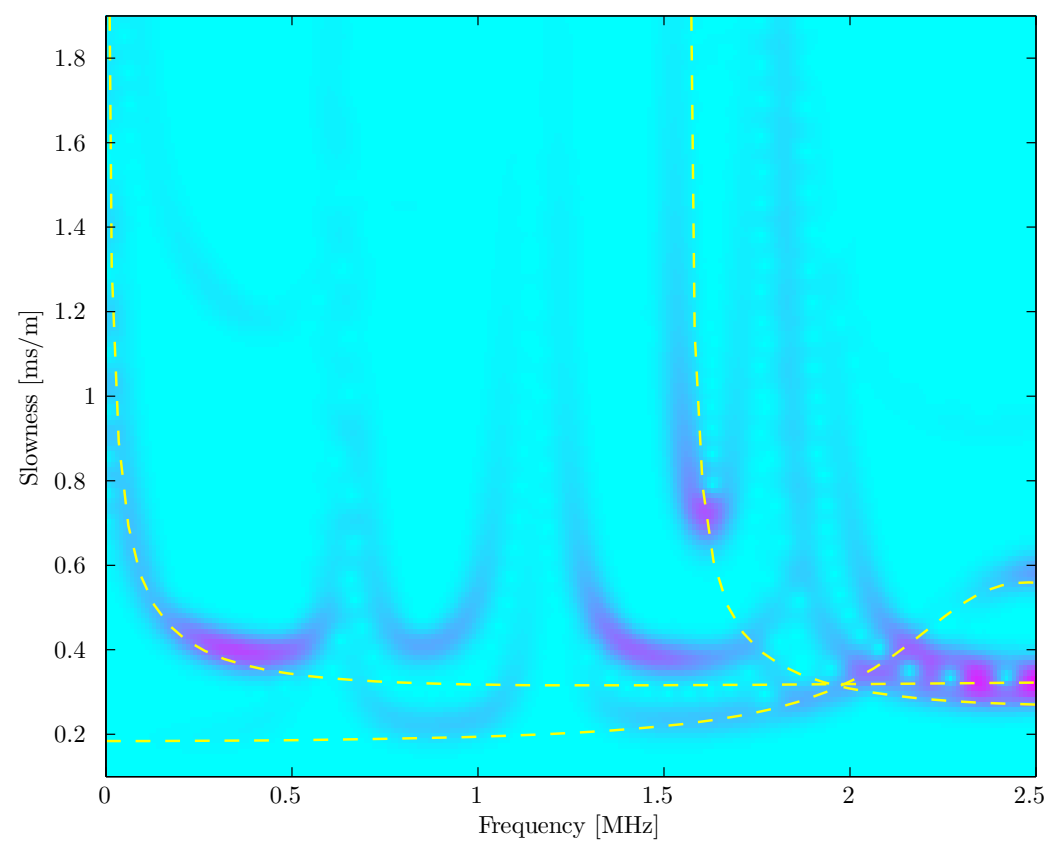

(a) In-plane component

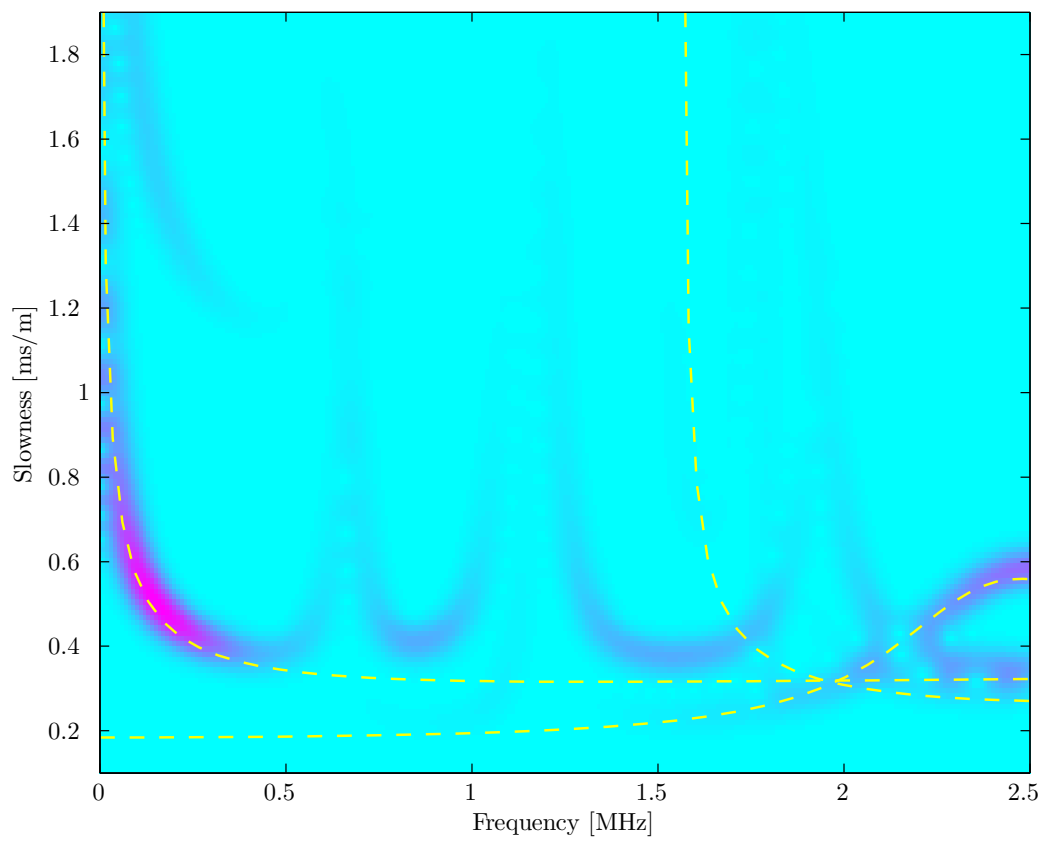

(b) Out-of-plane component

Figure 4.3: Slowness-frequency domain after the STFT of FE results for Alu-Tape2 with Alu modes (GMM) as yellow dashed lines. 


\subsection{Parametric studies (GMM)}

As a next step the GMM and the FEM are used to observe the effect of a change of one bond parameter at a time on the dispersion relations.

\subsubsection{Variation of the adhesive bond thickness}

Starting from the material properties of tape 2 (see Table 4.1) with the tape thickness $h_{t}=0.25 \mathrm{~mm}$ the first three Tape and Alu-Tape modes are investigated. Figures 4.4 and 4.5 show the first three modes for a tape thickness $h_{t}$ variation from $0.25 \mathrm{~mm}$ to $0.5 \mathrm{~mm}$ and $0.75 \mathrm{~mm}$. Although a variation of the adhesive bond thickness $h_{t}$ doesn't change the wave speeds $c_{L}$ and $c_{T}$ for the tape layer by itself, the influence on the Alu-Tape modes is significant. With decreasing $h_{t}$ the first mode stays longer under the influence of the A0 Alu mode. The same relation is valid for the second mode and the S0 Alu mode. For the second Alu-Tape mode and the A0 Alu mode as well as for the third and A0, S0 the influence zones shifts to higher frequencies with decreasing $h_{t}$. The influence zones in the frequency-wavenumber domain are the high slope parts where Alu and Alu-Tape modes are very close or even cross each other. In the slowness-frequency representation the influence zones are represented as local slowness minima close to the A0 and S0 Alu modes. In the latter representation, the shift is clearly visible for the second and third Alu-Tape mode in Figure 4.5. In other words an increase of $h_{t}$ in the double layered plate raises the influence of the single tape layer. Therefore the Alu-Tape modes follow the Alu modes less. Further on an increase of the tape thickness also means an increase of the number of modes in the same frequency-wavenumber window. The corresponding FE results show the same behavior like described in section 4.2 and can be found in Figures A.1 and A.2 of Appendix A. 

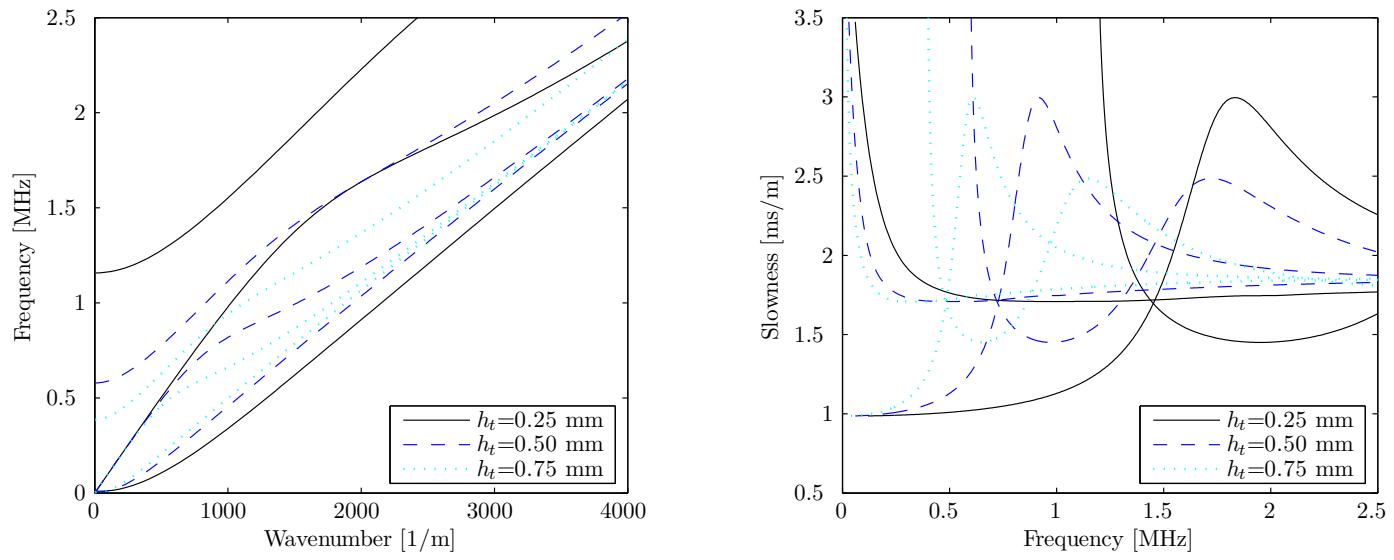

Figure 4.4: $h_{t}$ variation for Tape printed for the A0, S0 and A1 modes.
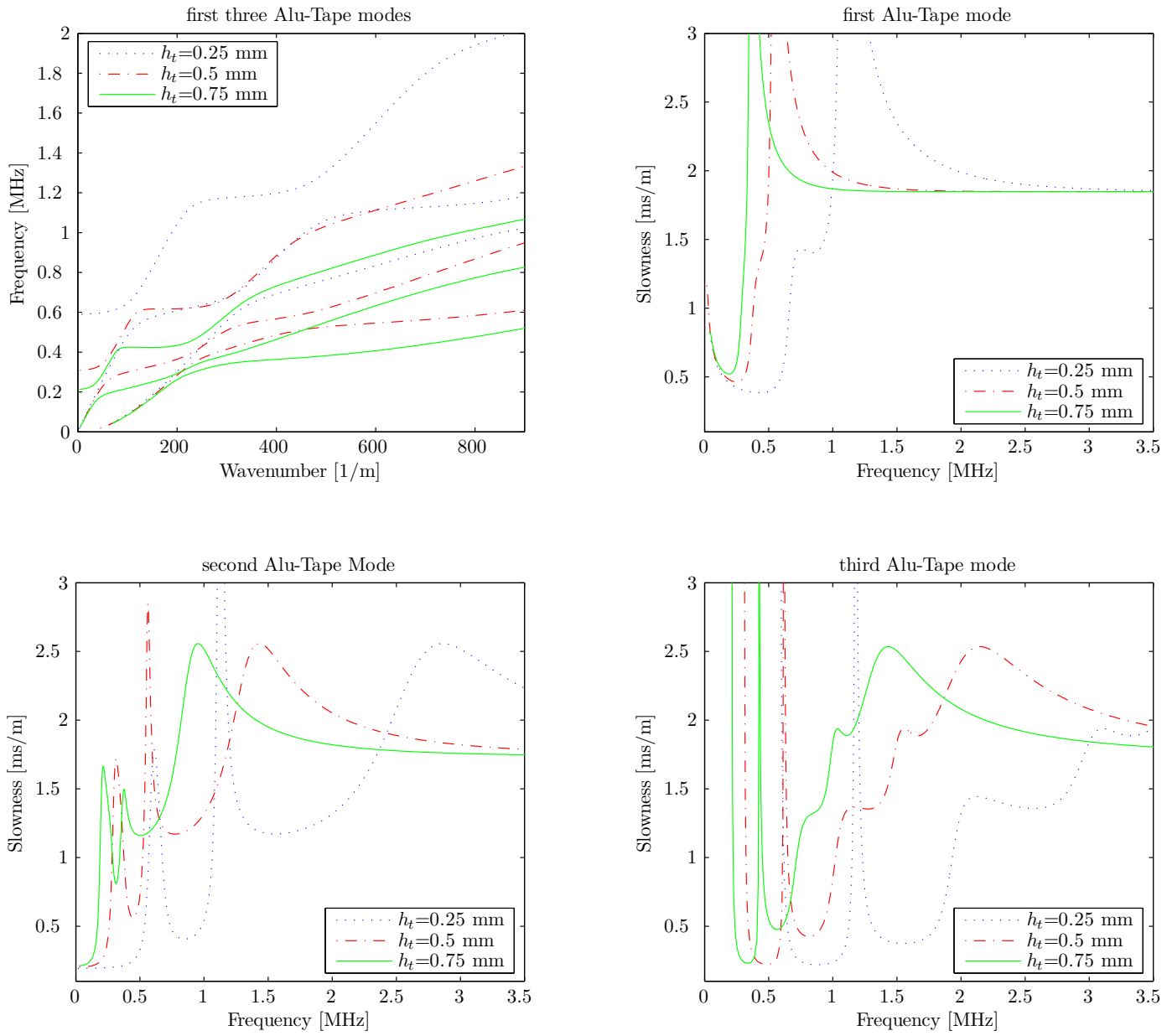

Figure 4.5: $h_{t}$ variation for Alu-Tape printed for the first three modes. 


\subsubsection{Variation of the Young's modulus}

Figure 4.6 pictures the first three modes of a single adhesive bond layer with the Young's modulus changing from $1.0 \mathrm{GPa}$ to $0.5 \mathrm{GPa}$ and finally to $0.25 \mathrm{GPa}$, while Figure 4.7 shows a change of $E_{t}$ in the same manner for the first three modes of a double layered plate. In contradiction to a tape thickness change, a variation of $E_{t}$ changes $c_{L}$ and $c_{T}$ in the Tape. Yet the effects on the dispersion relations look similar in the first moment. With increasing $E_{t}$ the influence zones shift to higher frequencies: the stiffer the Tape (the closer the Tape and the Alu are in terms of stiffness) the longer the combined modes follow the Alu-modes. The wave speeds for the Tape are higher for higher values of $E_{t}$. So it is possible to detect this change for example in the frequency-wavenumber domain (Figure 4.7) between the S0 and A0 Alu modes. In this region the slope of the second Alu-Tape mode increases with increasing $E_{t}$. This relationship is different for a change of $h_{t}$ where the slope stays the same. The according Figures A.3 and A.4 to this section for the FE results can be found in Appendix A.

\subsubsection{Variation of the Poisson's ratio}

In the last step the influence of the Poisson's ratio of the adhesive tape $\nu_{t}$ on the dispersion relations is examined. Figure 4.8 shows the first six Tape modes for a $\nu_{t}$ variation from 0.25 to 0.30 and 0.35 . According to those results, Figure 4.9 shows the first few Alu-Tape modes for three different $\nu_{t}$ variations. Due to the fact that the modes themselves don't change much, it becomes clear that the first two Alu-Tape modes don't change at all at low frequencies. One exception is beginning with the fourth Alu-Tape mode which changes to the third and second Alu-Tape mode after crossing the S0 and A0 Alu modes. The same behavior is visible in the slownessfrequency representation. So far the $\nu_{t}$ variation is only influencing the second gap 

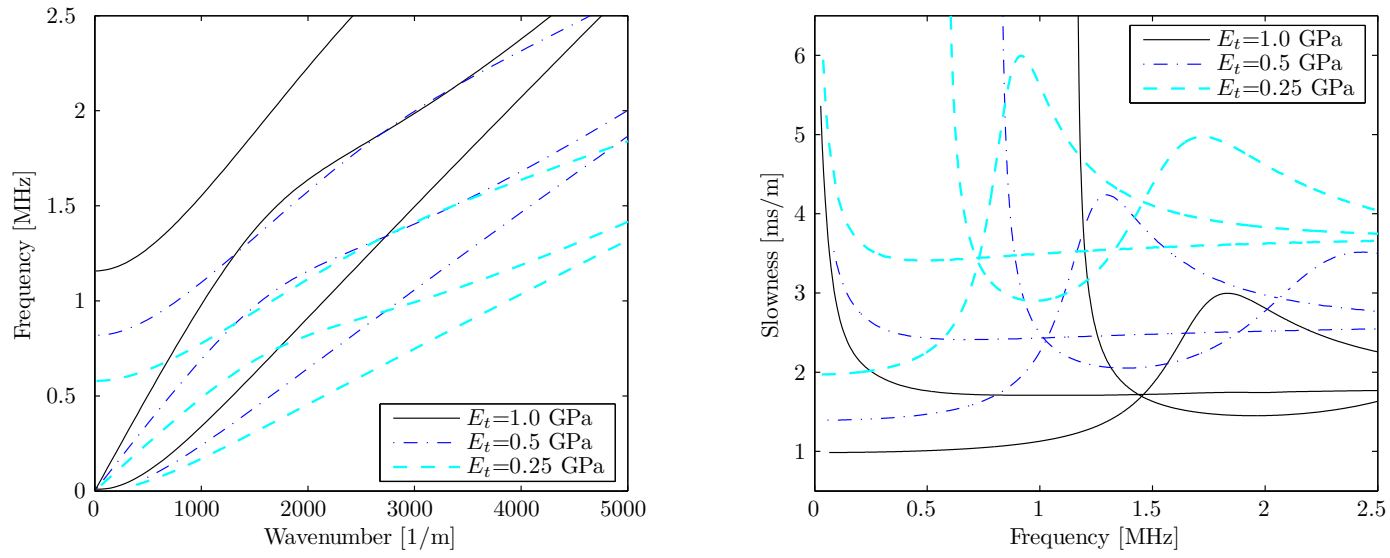

Figure 4.6: $E_{t}$ variation for Tape printed for the A0, S0 and A1 modes.
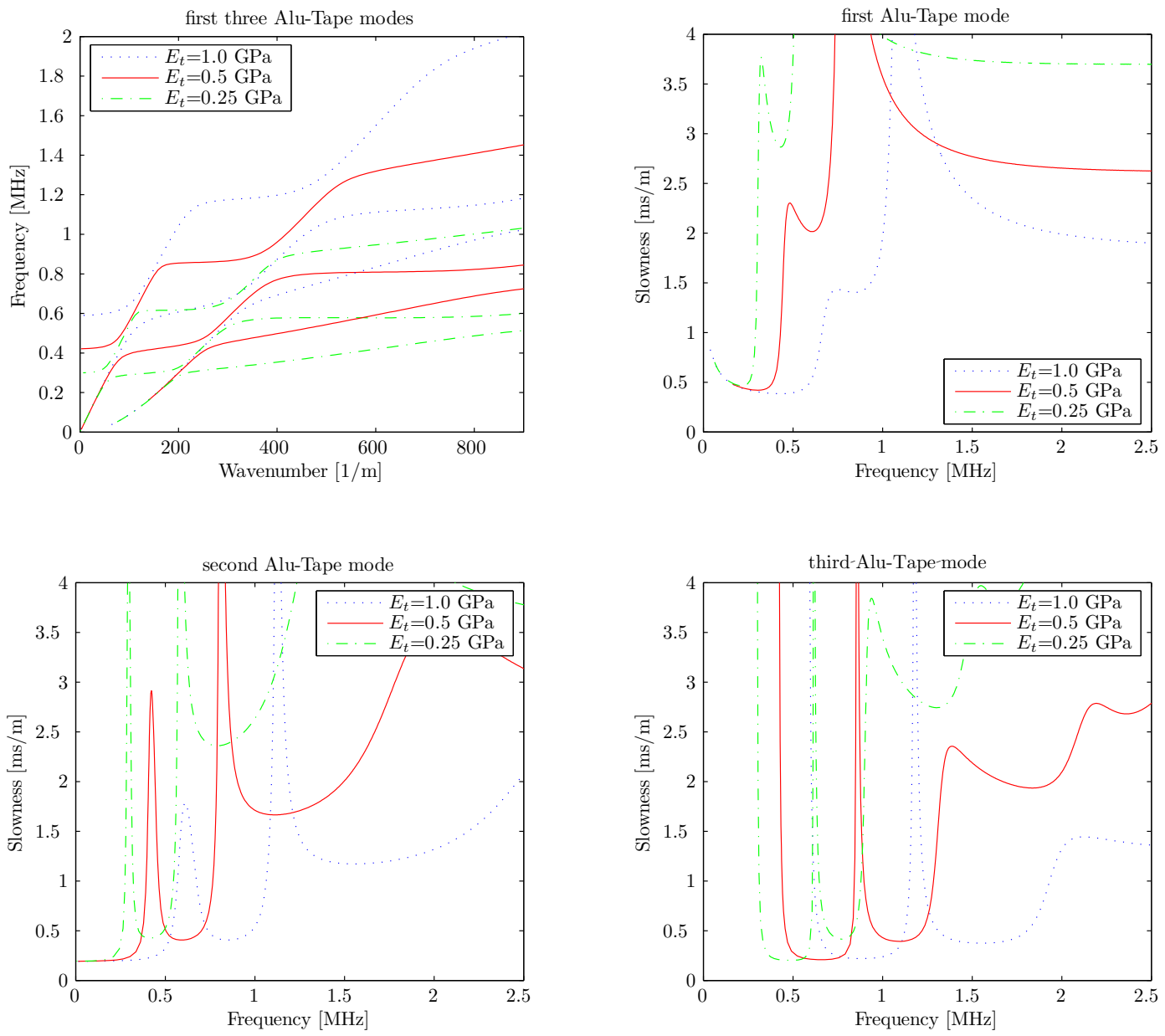

Figure 4.7: $E_{t}$ variation for Alu-Tape printed for the first three modes. 
along the A0 Alu mode and the third gap along the S0 Alu mode. In the slownessfrequency representation the slowness minima of the second Alu-Tape with A0 Alu mode as well as the third Alu-Tape with A0 and S0 Alu mode show an effect of the $\nu_{t}$ variation. The same statements are possible for the according FEM results represented by Figures A.5 and A.6 in Appendix A.
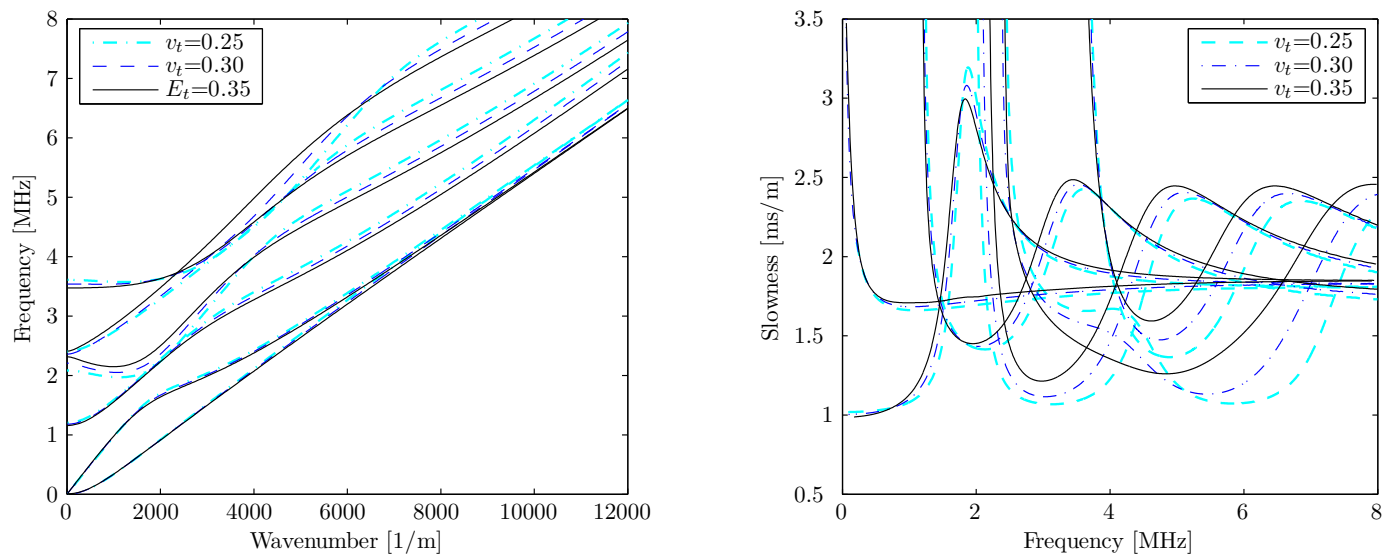

Figure 4.8: $\nu_{t}$ variation for Tape printed for the A0 to A2 modes.
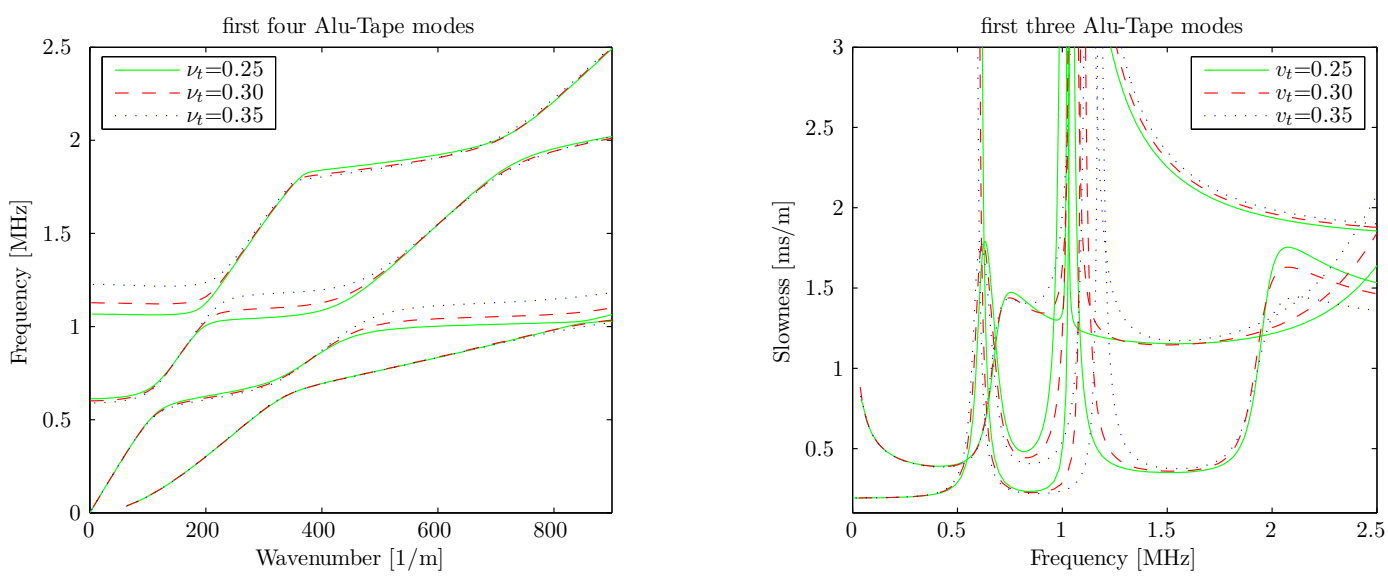

Figure 4.9: $\nu_{t}$ variation for Alu-Tape printed for the first few modes. 


\subsection{Typical waveguide $F E$ (WFE) results}

As a last step the waveguide FE method is used for an Alu-Tape plate, where $l_{e}$ is $0.02 \mathrm{~mm}$ for the aluminum and $0.01 \mathrm{~mm}$ in the tape layer. Figure $4.10(\mathrm{a})$ shows the frequency-wavenumber relation for this 75 element model while Figure 4.10(b) shows the slowness-frequency relation. Due to the lower transversal wave speed of the tape the accuracy window decreases compared to the single aluminum plate case from Section 3.3.4 (if the same $l_{e}$ would be assumed). Otherwise the equations responsible for the accuracy window $((3.45)$ to $(3.47))$ are also valid for the double layered plate.

A clear advantage of this method compared to the global matrix method is the ability to create the dispersion curves of any periodic structure and not only of layered plates. For example in [7] a rippled plate and a fluid filled corrugated pipe are investigated. However, the accuracy is influenced by the element size and the material properties, and the computational costs are influenced by the mesh size. The use of materials with very low transversal wave speeds causes a very small accuracy window. To correct this problem a smaller element size could be used, which would lead to a much longer calculation time. This is especially critical in this case, where adhesive bonds with a very low stiffness are used. The global matrix method on the other side also shows numerical difficulties for materials with very low wave speeds and more than two layers. Finally, from an inversion point of view, the interface between the FE-software and the optimization software presents an obstacle and is the reason why this method is not used in the inversion process of this work. 


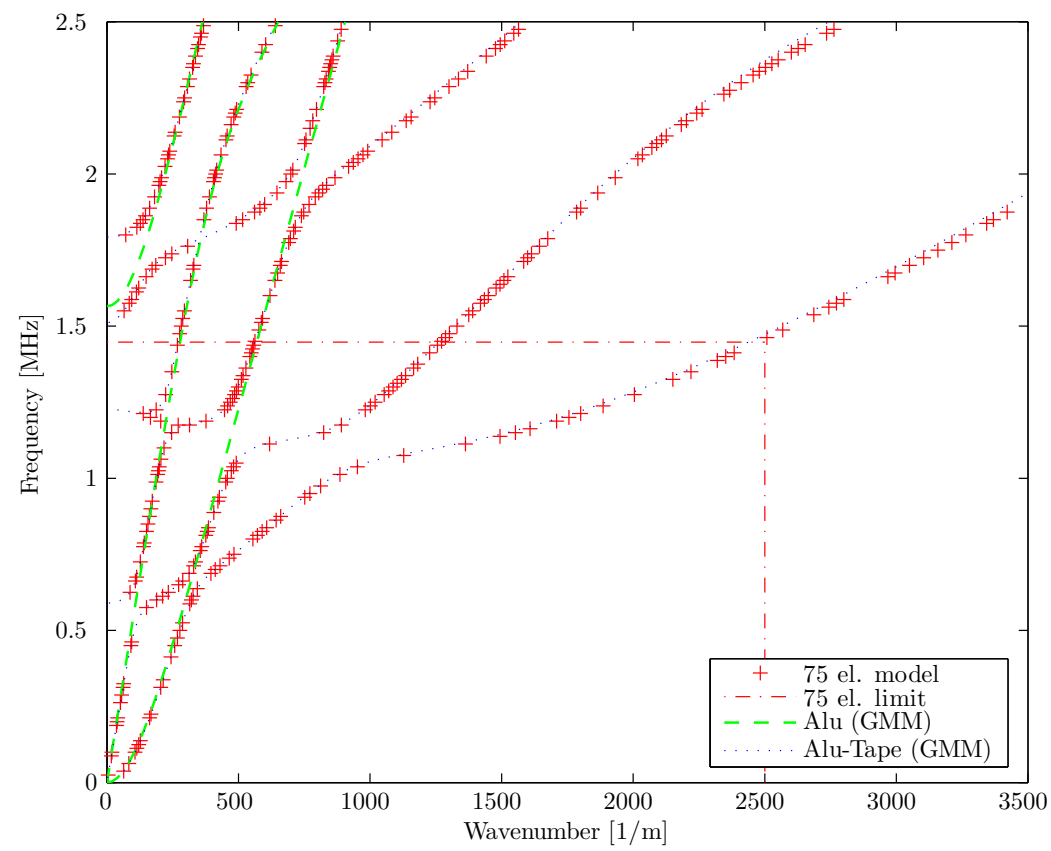

(a) Frequency-wavenumber relation

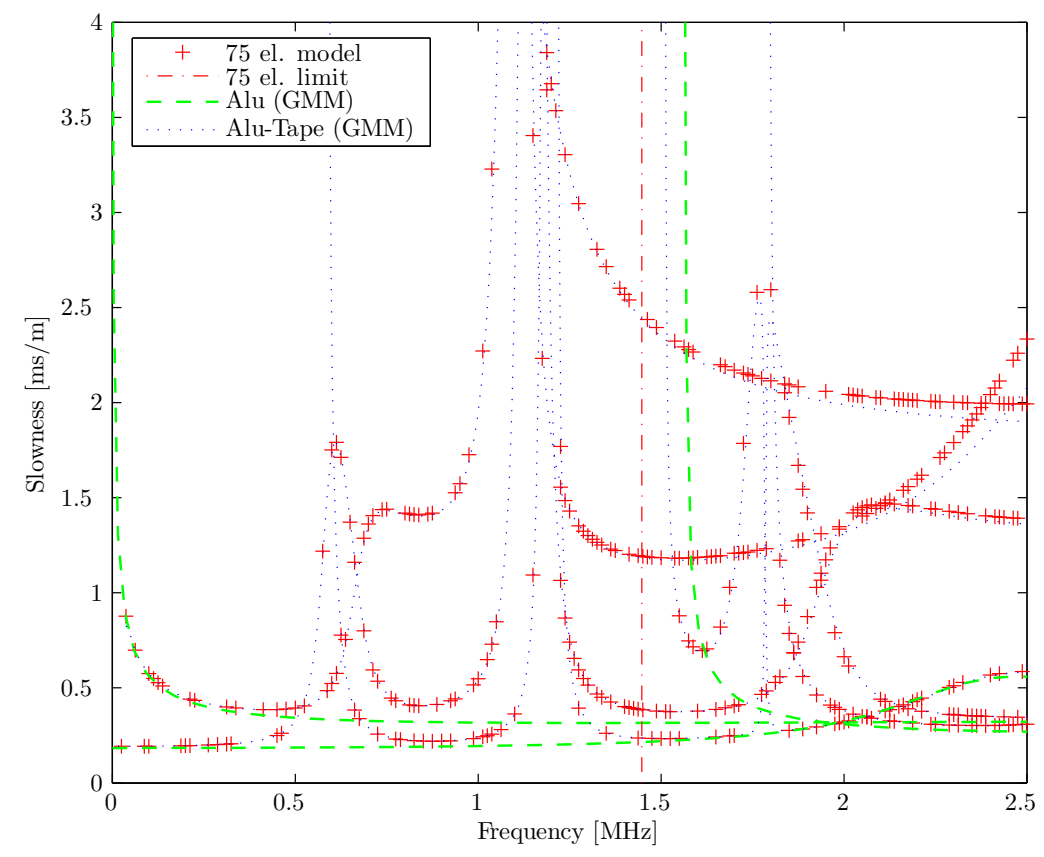

(b) Slowness-frequency relation

Figure 4.10: Waveguide FE results for a 75 element double layered Alu-Tape plate. 


\section{CHAPTER V}

\section{CONNECTING FORWARD TO INVERSE PROBLEM}

So far three possibilities to solve the forward problem are presented: the global matrix method, the FE simulation and the waveguide FE method. Depending on the experiment to be simulated, a frequency-wavenumber representation or a slownessfrequency representation is obtained. Considering the experimental costs, producing one transient output signal is the "easy" way. In order to solve the inverse problem a few parameter sensitive and FE-simulation-visible points have to be chosen as an input for the inversion procedure. Although the preceding chapter already shows tendencies as to choose these points, this process is iterative and offers lots of different possibilities. Due to the FE results and the fact that the dispersion relation of the single layer of aluminum is known the following sets of sensitive points are chosen.

\subsection{Frequency-wavenumber representation}

As seen in Section 4.3 the crossing points and the gaps of the Alu-Tape modes with the Alu modes represent a sensitive and visible set of important points. In this case the wavenumber or the frequency of those points could be chosen to define them. In other words a projection of the FE results along any Alu mode on the frequency or wavenumber axis could be represented as a function where the maxima represent the crossing points and the minima represent the gaps. Figure 5.1 shows this relation. After comparing the results of this procedure along the first three Alu modes it becomes clear that the A0 Alu mode for in-plane FE data delivers the best results. Therefore all further investigations are based on a projection of the 2D-FFT results 
(in-plane FE data) along the A0 single layer aluminum mode.

Due to the 3D nature of the 2D FFT results of an FE simulation or experiment the minima for this procedure cannot be achieved with other methods like the GMM or WFE. These methods represent the frequency as function of wavenumber for every single mode, which would only produce the maxima for the so far used procedure. Therefore the procedure to find the minima has to be same for the forward problem and for the inverse problem. A closer look at this issue is presented in the chapter for the actual inversion.

\subsection{Slowness-frequency representation}

In this representation the points that are sensitive to a tape parameter change and visible have to be found, too. According to the parametric studies and the fact that the A0 Alu mode is considered, the choice fell on the slowness minima of the AluTape modes that are closest but above the A0 Alu mode, see Figure 5.1. According to equations (2.20) and (2.21) for the absence of attenuation those slowness minima in the slowness-frequency representation are the points of maximum slope for each Alu-Tape mode crossing the Alu mode in the frequency-wavenumber representation. 


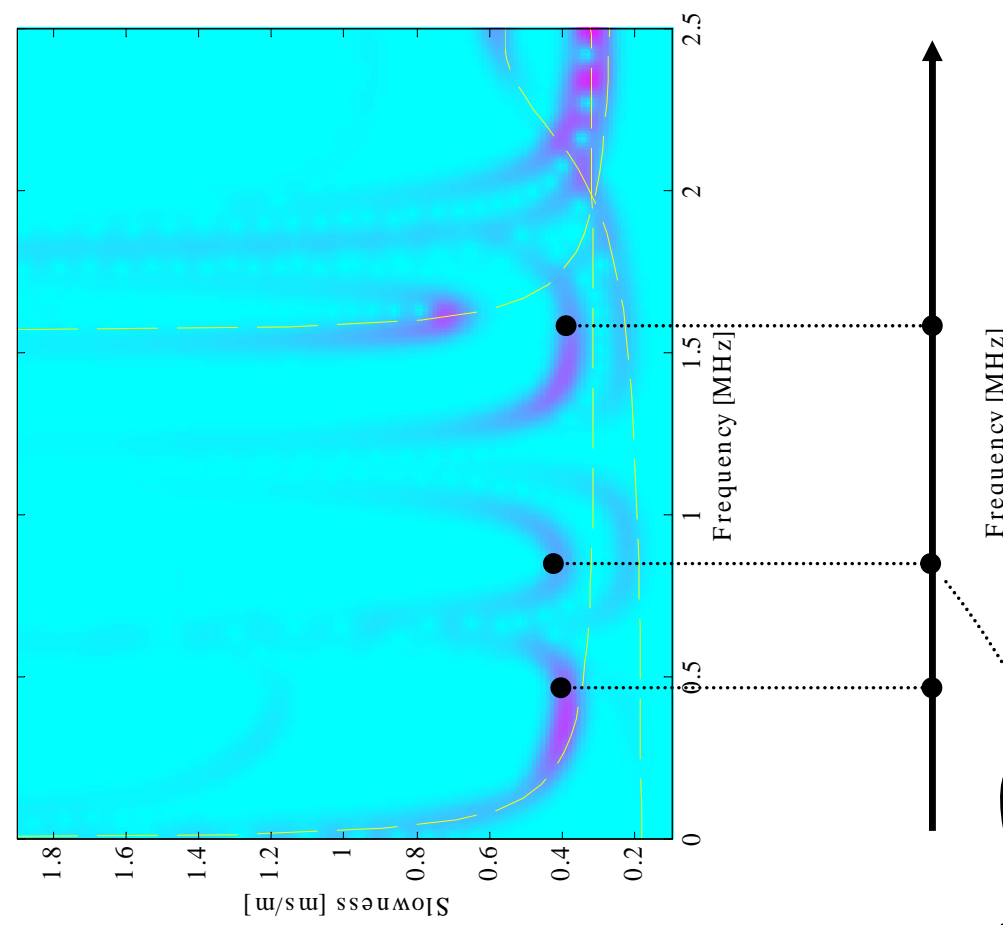

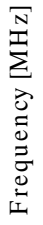

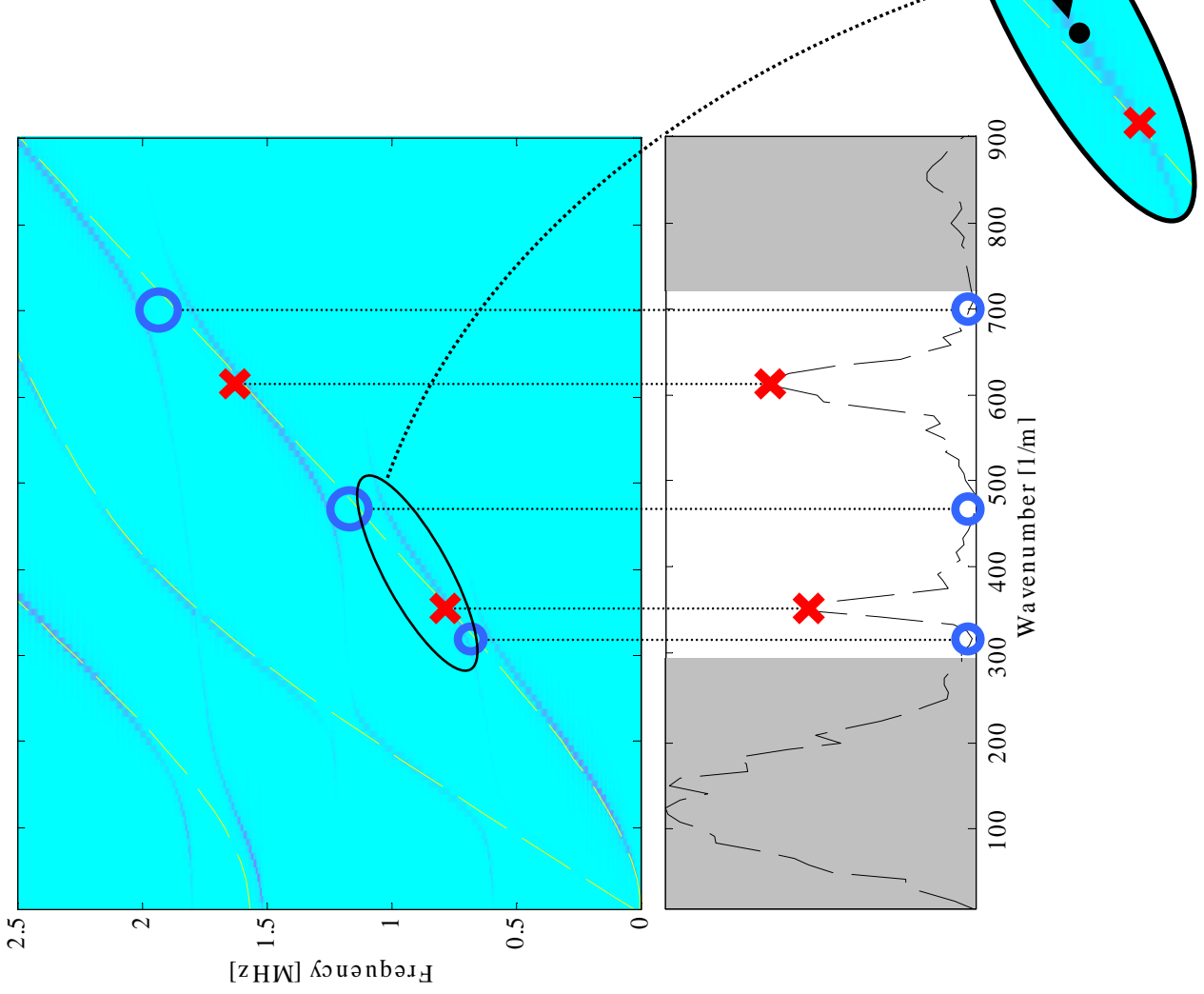

Figure 5.1: Relation of the sensitive points between the different representations. 


\subsection{Adhesive bond parameter influence}

Any change in the tape parameters causes a "shift" of the combined Alu-Tape modes with respect to the single aluminum plate modes. Therefore the tape parameters also influence the frequency location of the minima, maxima and points of maximum slope. In order to determine if the selected points are suitable for an inversion procedure, the influence of the bond parameters on the frequency location of those points is observed. Therefore one parameter at a time is changed and the changes are examined.

Figure 5.2 shows first a variation of the tape thickness $h_{t}$ from $0.25 \mathrm{~mm}$ to 0.5 $\mathrm{mm}, 0.75 \mathrm{~mm}$ and $1.0 \mathrm{~mm}$. This figure represents the expected behavior from Section 4.3. With increasing $h_{t}$ all points shift to lower frequencies. Although the observed movement with changing $h_{t}$ is similar for all points, it is not linear. Remarkable is the fact that the sequence of the points stays the same except for the crossing point and the point of maximum slope for the third Alu-Tape mode, which swap positions.

The second figure shows a variation of the Young's modulus of the tape $E_{t}$ from 1.0 GPa to $0.75 \mathrm{GPa}, 0.5 \mathrm{GPa}$ and $0.25 \mathrm{GPa}$. With increasing $E_{t}$ all points shift to higher frequencies and stay in the same sequence.

The third and last figure shows a variation of the Poisson's ratio of the tape $\nu_{t}$ from 0.25 to $0.3,0.35,0.4$ and 0.45 . The effect of this change on the points is quite different. The first point of maximum slope, the minimum between the first and second AluTape mode and the crossing points of the second and third Alu-Tape mode stay almost at the same frequencies while the other points move to higher frequencies with increasing Poisson's ratio. The crossing point and the point of maximum slope for the third Alu-Tape mode swap positions. This will be crucial for the inversion process and presents the reason why an inversion based on multiple transient output signals is capable to perform a three parameter optimization. 

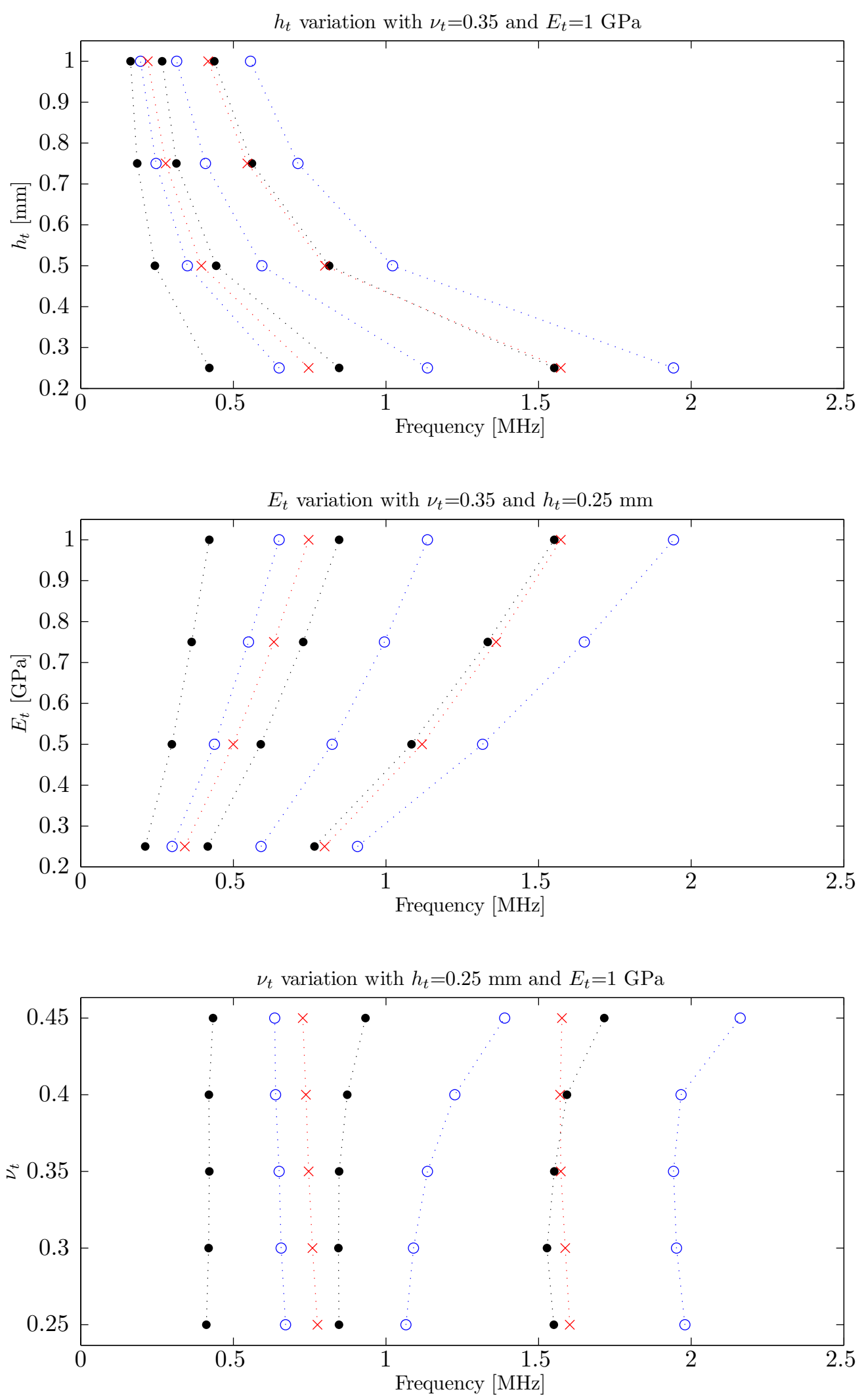

Figure 5.2: Influence of a variation of one parameter at a time on the minima (o), maxima (x) and points of maximum slope $(\bullet)$. 


\section{CHAPTER VI}

\section{INVERSE PROBLEM}

So far the forward problem is solved with the FEM Simulation in order to model a real experiment. Further on trends have been observed and based on that a set of critical points have been selected, whose frequency locations serve as the input for the inversion procedure. Figure 6.1 summarizes the complete procedure of the inverse problem. Important parts herein are the algorithm that uses the global matrix method to back out the necessary sensitive points, the error-function and the optimization algorithm.

The inversion procedure is applied on two cases. Just like stated in the previous chapter it would be preferable from an experimental point of view to work with only one transient output signal. Therefore first the points of maximum slope are

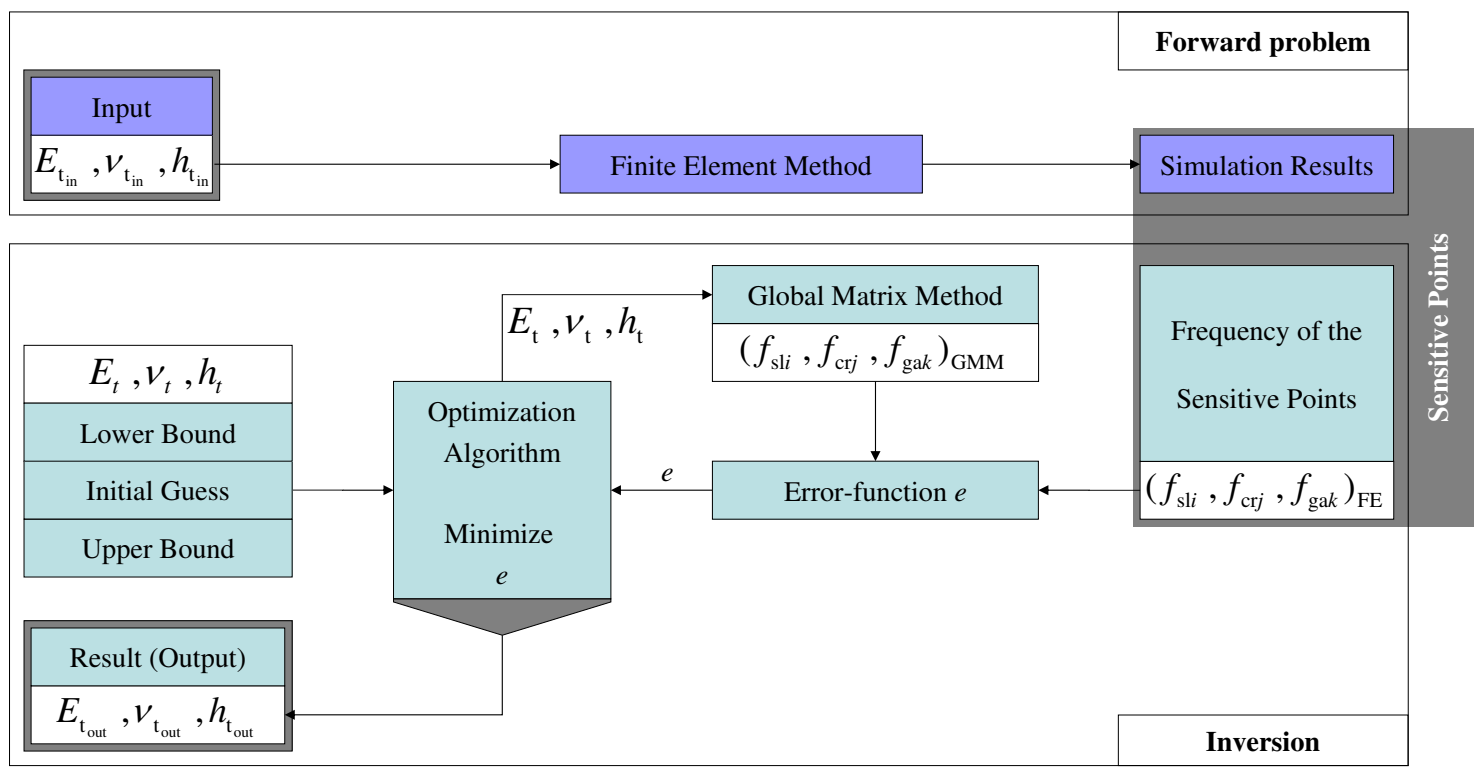

Figure 6.1: Structure of the forward and inverse problem. 
considered as an input for the inversion procedure. After that the inversion procedure is applied on the results of a simulation with multiple output points, which allows all so far identified sensitive points as an inversion input. Afterwards a weaker adhesive bond and a triple layered plate are discussed.

\subsection{Parts of the inversion process}

In this section important parts of the inversion procedure will be explained. First the algorithm that uses the global matrix method to back out the necessary sensitive points is described. After that the role of the error-function is presented and finally some facts about the used optimization algorithm are identified.

\subsubsection{Computing the sensitive points with the GMM}

During one optimization depending on the optimization algorithm up to 300 function evaluations are performed. Therefore the speed of the algorithm that uses the global matrix method to back out the necessary sensitive points is crucial. The biggest influence on the computation time of the global matrix method are the step sizes

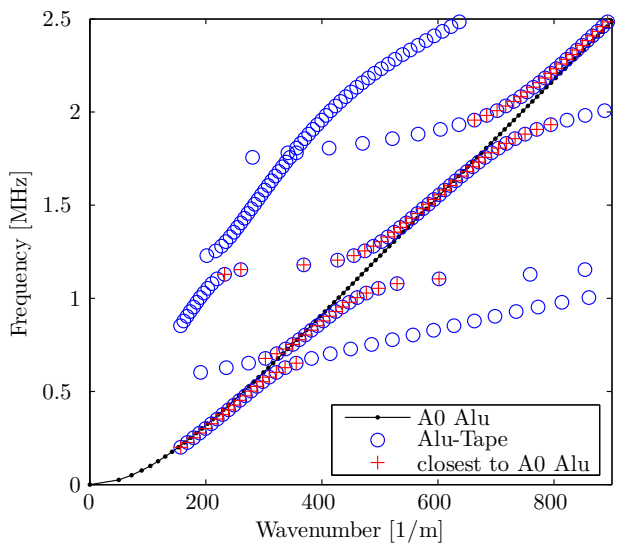

(a) closest points to A0 Alu

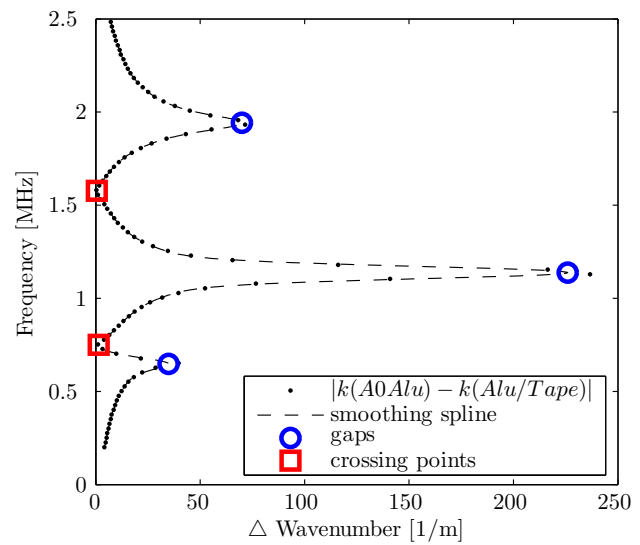

(b) $|\triangle k|$ between A0 Alu mode and closest Alu-Tape solutions

Figure 6.2: Result of the global matrix method for Alu-Tape and Alu. 


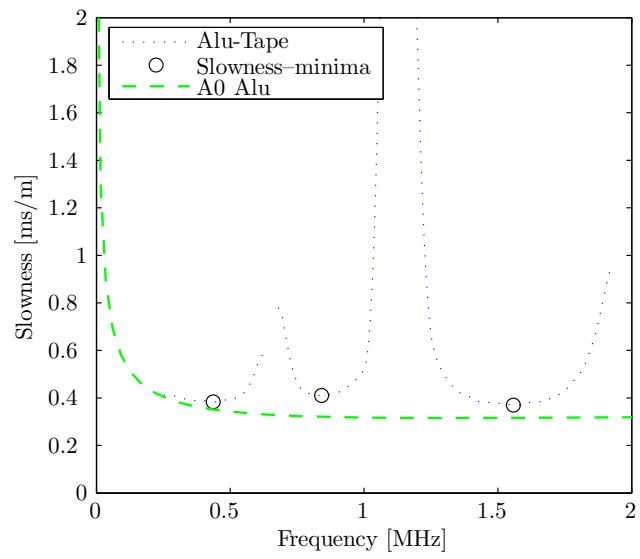

Figure 6.3: Slowness-minima for the first three Alu-Tape modes closest to the A0 Alu mode.

of the frequency and the wavenumber in the scanning approach. Therefore an approximation based on a previously stored set of 125 values ( 5 steps for each material parameter) in between the upper and lower bounds restrict the calculation range in terms of frequency and wavenumber. For the tape parameters of Tape2 for example 100 frequency steps and 2400 wave number steps are made. After the calculation via the global matrix method is finished in the approximated frequency and wavenumber ranges, for each frequency the closest point in terms of wavenumber distance to the actual A0 Alu mode is selected, see Figure 6.2(a). The wavenumber of all these closest points is then a function of frequency. This function is continuous for every single Alu-Tape mode and shows a discontinuity in between two modes. In the next step the absolute value of the difference of this function and the A0 Alu mode (of the same frequency) in terms of wavenumber is computed and approximated by a smoothing spline. Figure 6.2(b) shows the new created function which has a minimum at each frequency location where a crossing point exists and a maximum at each frequency location where a gap would be centered. In other words a gap is located at that frequency which has a maximal wavenumber difference to the closest solution. The procedure to find the minima of the 2D-FFT results from a simulation or an experiment has to be same. 
In the next step the first three continuous functions (representing the first three Alu-Tape modes) are separated using the frequency location of the gaps. Each function is fitted with a smoothing spline and equations (2.20) and (2.21) lead to the slowness-frequency representation. In this representation (Figure 6.3) the slowness minima of each function represent the point of maximum slope in the frequencywavenumber representation, as explained in Section 5.2.

\subsubsection{Error-function $e$}

The optimization algorithm tries to minimize the error-function. The error-function is set up to quantitatively describe how well the result of the global matrix method matches the frequency location of the prior selected sensitive points from the FE simulation. Therefore an error-function $e$ which would use all so far identified points for example is

$$
\begin{aligned}
e & =\sum_{i=1}^{3}\left|\left(f_{\mathrm{s} l i}\right)_{\mathrm{GMM}}-\left(f_{\mathrm{s} l i}\right)_{\mathrm{FE}}\right| \\
& +\sum_{j=1}^{2}\left|\left(f_{\mathrm{cr} j}\right)_{\mathrm{GMM}}-\left(f_{\mathrm{cr} j}\right)_{\mathrm{FE}}\right| \\
& +\sum_{k=1}^{3}\left|\left(f_{\mathrm{ga} k}\right)_{\mathrm{GMM}}-\left(f_{\mathrm{gak}}\right)_{\mathrm{FE}}\right|
\end{aligned}
$$

where "sl" denotes the points of maximum slope, "cr" the crossing points and "ga"

the gaps. The maximum set of sensitive points along the A0 Alu mode consists of three points of maximum slope, two crossing points and three gaps. Sections 6.2.1 and 6.2.2 explain the choice of critical points in detail.

\subsubsection{Optimization algorithm}

Optimization problems can take many iterations to converge and can be sensitive to numerical problems. Moreover, there is no guarantee that the optimization algorithm finds the global minimum. Different algorithms have been used to verify the results and to find the solution to the inverse problem in an efficient way. 
The present problem is in the category of constrained optimizations. The used algorithm is for medium scale problems, which means that it is based on a line search as opposed to large scale algorithms which use trust regions. Further on the algorithm is based on the quasi-Newton method and uses a sequential quadratic programming (SQP) routine. SQP methods represent the state of the art in nonlinear programming methods. The method offers the possibility to closely mimic Newton's method for constrained optimization just as is done for unconstrained optimization. At each major iteration, an approximation is made of the Hessian of the Lagrangian function using a quasi-Newton updating method. This is then used to generate a quadratic programming (QP) subproblem whose solution is used to form a search direction for

a line search procedure. For further reference the interested reader should consult $[4,12]$.

\subsubsection{Initial guess, lower and upper bounds}

Starting the optimization from a number of different starting points helps to locate the global minimum. In this case four different starting points (initial guesses) are used to find a solution and validate the result. A set of results consisting of the three result parameters $E_{t}, \nu_{t}$ and $h_{t}$ is only a valid result if the value of the error-function $e$ reaches a minimum relative to the other three results. In order to validate the capabilities of the procedure and the chosen set of sensitive points the deviation sum also has to be a minimum compared to the other three deviation sums. The deviation sum is the sum of the deviation of each parameter. The deviation $\sigma_{E_{t}}$ for the Young's modulus of the adhesive bond $E_{t}$ for example is the relation of the result value $E_{t_{\text {out }}}$ of the inversion to the input value $E_{t_{\text {in }}}$ of the forward problem

$$
\sigma_{E_{t}}=\frac{\left|E_{t_{\mathrm{out}}}-E_{t_{\mathrm{in}}}\right|}{E_{t_{\mathrm{in}}}} .
$$

The input values for the forward problem are those of Table 4.1. The upper and lower bounds in Table 6.1 are chosen for different reasons. One reason is the investigated 
Table 6.1: Starting points, lower and upper bounds for the inversion.

\begin{tabular}{|l|c|c|c|}
\hline Tape & $E_{t}[\mathrm{GPa}]$ & $\nu_{t}$ & $h_{t}[\mathrm{~mm}]$ \\
\hline Lower bound & 0.25 & 0.27 & 0.1 \\
\hline Initial guess 1 & 0.5 & 0.3 & 0.5 \\
\hline Initial guess 2 & 0.5 & 0.4 & 0.5 \\
\hline Initial guess 3 & 1.2 & 0.3 & 0.5 \\
\hline Initial guess 4 & 1.2 & 0.4 & 0.5 \\
\hline Upper bound & 1.25 & 0.44 & 0.95 \\
\hline
\end{tabular}

range of parameter values in Section 5.3, so that concrete statements are possible. On the other side the GMM algorithm has to be time efficient, because many inversions, each with four initial guesses on the values of $E_{t}, \nu_{t}, h_{t}$ and up to 300 function evaluations, have to be calculated. Another reason for the values of the bounds is the fact that the algorithm has to be $100 \%$ stable inside those bounds. Stability and time efficiency create a compromise that is represented in the choice of the upper and lower bound. The four initial guesses can also be found in Table 6.1. A small change of $h_{t}$ has usually a relative big influence (see Figure 5.2) on the value of the error-function, that's why a variation of $h_{t}$ for the initial guesses is unnecessary.

\subsection{Results}

Computing the frequency location of the critical points with the FEM and using the GMM to find the tape parameters based on those values makes the deviation between both a very critical subject. Table 6.2 shows all so far identified sensitive points and their frequency location obtained with the FEM and with the GMM. Further on the frequency difference and deviation for each point is presented. The deviation for every single point stays below $1 \%$, although both methods are influenced 
Table 6.2: Frequency locations of the critical points.

\begin{tabular}{|l|c|c|c|c|}
\hline Point & GMM $[\mathrm{kHz}]$ & FEM $[\mathrm{kHz}]$ & $\triangle f[\mathrm{kHz}]$ & Deviation $[\%]$ \\
\hline$f_{\text {sl1 }}$ & 422.5 & 420 & 2.5 & 0.59 \\
\hline$f_{\text {ga } 1}$ & 648.0 & 651 & 3.0 & 0.46 \\
\hline$f_{\text {cr1 }}$ & 749.8 & 756 & 6.2 & 0.82 \\
\hline$f_{\text {s12 }}$ & 852.0 & 850 & 2.0 & 0.23 \\
\hline$f_{\text {ga2 }}$ & 1137.4 & 1140 & 2.6 & 0.23 \\
\hline$f_{\text {sl3 }}$ & 1555.5 & 1560 & 4.5 & 0.29 \\
\hline$f_{\text {cr2 }}$ & 1577.2 & 1570 & 7.2 & 0.46 \\
\hline$f_{\text {ga3 }}$ & 1942.4 & 1950 & 7.6 & 0.39 \\
\hline
\end{tabular}

by numerical noise. A too high frequency difference increases the probability that an other parameter combination is at the global minimum. Therefore all inversion processes are also tested with GMM results in which case the global minimum is guaranteed to be at the right parameter combination.

\subsubsection{Case of only one transient output signal}

Using only one transient output signal provides only the points of maximum slope as an input for the optimization (inversion) process. Due to this and the fact that a change in Youngs modulus $E_{t}$ or tape thickness $h_{t}$ have a similar influence on the frequency location of the points of maximum slope, a full three variable optimization is not possible. This becomes clear in the optimization results where the set of result parameters with the smallest deviation sum does not have the smallest value for the error-function. This behavior can be observed if the set of sensitive points consists of all three slowness minima or just of the last two slowness minima. Even if the forward 

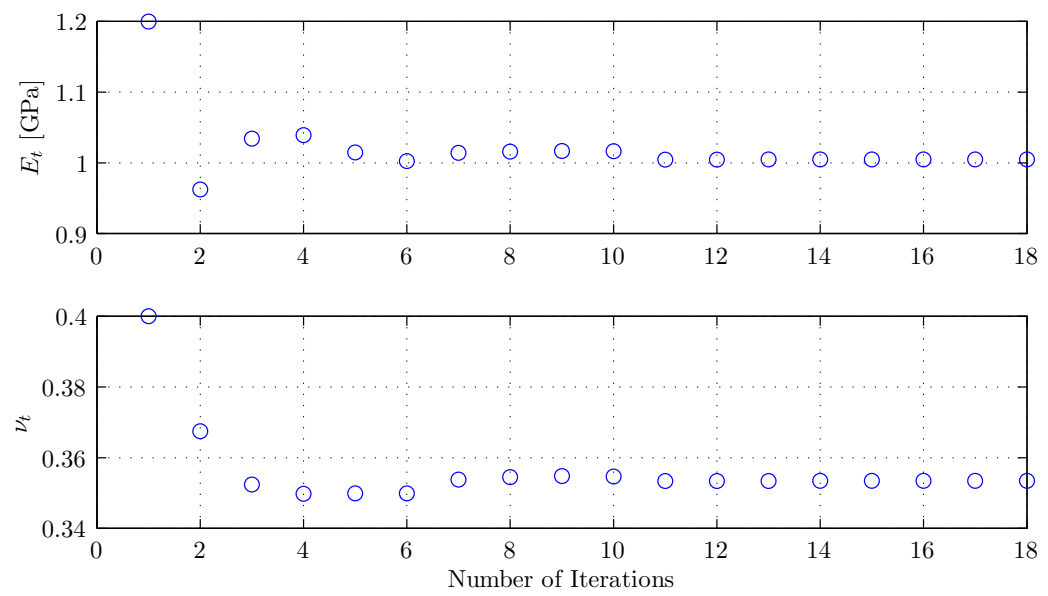

Figure 6.4: Two parameter optimization path for $f_{\mathrm{sl} 2}$ and $f_{\mathrm{sl} 3}$ of FEM results.

problem is solved with the GMM instead of the FEM the inversion procedure is not capable of performing a three parameter optimization.

This limits the case of only one transient output signal to a two parameter optimization. Therefore $h_{t}$ is assumed to be known. The best results are obtained if only the second and third slowness minima of the FEM results are used. For $E_{t_{\text {out }}}=10.0496 \mathrm{GPa}$ and $\nu_{t_{\text {out }}}=0.3535$ the deviation sum is only $1.5 \%$. Figure 6.4 shows the path of this result. Although the figure shows only 18 iteration steps the process has more than 200 function evaluations.

\subsubsection{Case of multiple transient output signals}

Using multiple transient output signals provides all three determined sets of sensitive points as an input for the optimization process. Therefore a full three variable optimization is possible. This is so because the behavior for a change in $E_{t}$ or $h_{t}$ can be differentiated if more than only the points of maximum slope are used. Considering this statement and Figure 5.2 especially the use of the second maximum should lead to good results. Using the second and third point of minimum slope and the second crossing point leads to a deviation sum of $7.2 \%$ for the FEM forward problem. Figure 6.5 pictures the according inversion path with the final values $E_{t_{\text {out }}}=9.586$ 

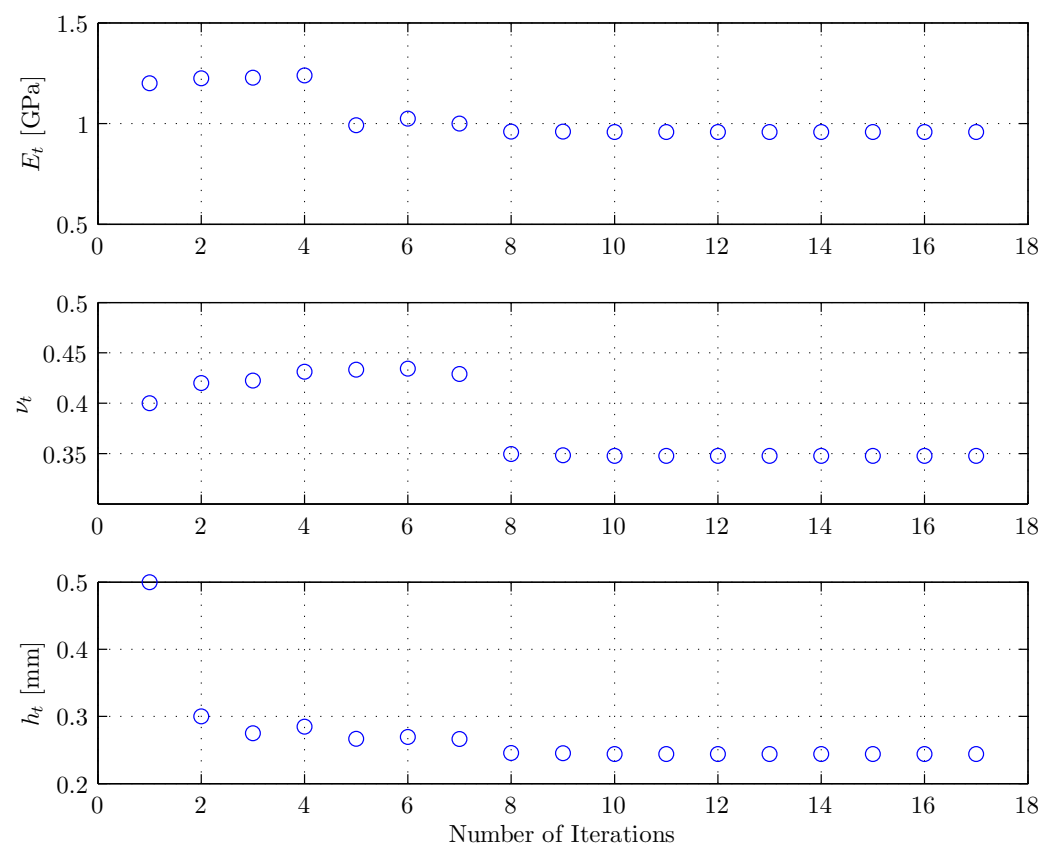

Figure 6.5: Three parameter optimization path for $f_{\mathrm{sl} 2}, f_{\mathrm{sl} 3}$ and $f_{\mathrm{cr} 2}$ of FEM results.

$\mathrm{GPa}, \nu_{t_{\mathrm{out}}}=0.3477$ and $h_{t_{\mathrm{out}}}=0.2441 \mathrm{~mm}$. A three parameter optimization is much harder than the inversion for only two parameters. All presented results are heavily dependent on the environmental settings (step sizes, lower and upper bounds, fitting tools, resolution of FE data) for the algorithms. Hence a sensitivity for numerical noise in the GMM algorithm has the ability to disturb the optimization algorithm. This also means that the presented results could be improved with an upgraded setup, fine tuning or more computation power. Further on the surface impedance matrices of [8] might offer a possibility to create the dispersion relation in a more time efficient way. Another possibility to reduce calculation time would be to track for example only the second Alu-Tape mode with an eigenpath analysis [11]. This would lead to one influence zone on the S0 Alu mode and one influence zone on the A0 Alu mode. Higher modes would show more influence zones but are also more likely to be damped out $[20]$. 


\subsection{Limits and outlook}

The inversion procedure for a double layered plate using an adhesive bond with a relative high stiffness has been investigated so far. Now a second adhesive bond with a relatively low stiffness as well as an adhesive bonded triple layer plate are investigated.

\subsubsection{Lower stiffness adhesive bond}

Figures 6.6 and 6.7 show the results of an FE simulation for a double layered plate using tape 1 called "Alu-Tape1". Tape 1 has a significantly lower stiffness than tape 2 while the Poisson's ration is very high, see Table 4.1. The number of modes in the same frequency-wavenumber window increases. Alu-Tape2 shows three and a half modes along the A0 Alu mode up to $2.5 \mathrm{MHz}$ while Alu-Tape1 shows five in the same frequency range. The parts of low slope don't even show up. Hence it's possible to say that the Tape1 influence on the Alu-Tape1 modes is very small. The stiffness difference becomes also clear if the transition zones are compared. A transition zone is the area on both sides of an Alu mode where the influence of the Alu on the Alu-Tape modes vanishes, in other words where the Alu-Tape mode changes from a generally low slope to a higher slope that is comparable with the slope of the Alu mode. The transition zones are much more distinct for Alu-Tape2, they almost vanish at higher frequencies for Alu-Tape1.

All those differences between Alu-Tape1 and Alu-Tape2 influence the possibility of an inversion procedure. The inversion will be much harder for a Alu-Tape plate with Tape1 as an adhesive bond. One reason is that the crossing points are hard to identify because the Alu-Tape modes and the Alu modes almost have the same slope in the higher slope parts. The gaps are clearly visible in the A0 Alu mode for the in-plane velocity component. The slowness minima are also present although numerical noise could cause variations in the inversion results. 
Even though the inversion of Alu-Tape1 seems to be feasible for FE results it is also an example of the limits of the inversion procedure. If properties of the adhesive bond and the adherent are too different an inversion as suggested in this work becomes more and more difficult.

\subsubsection{Triple layered plate}

The investigation of double layered structures was successful so far. Triple layered structures though have more application possibilities. Figures 6.8 and 6.9 depict the FE results for a triple layered plate using Tape2 to connect two aluminum plates. Both representations look different from the double layer Alu-Tape2 figures (4.2 and 4.3). The main difference is the fact that always two modes follow one Alu mode, while there is only one mode following an Alu mode for the Alu-Tape. The number of modes for example along the A0 Alu mode almost stays the same, but the AluTape modes follow the Alu mode generally longer than for the two layered plate. For example the frequency for the first mode to leave the A0 Alu mode is around $1.1 \mathrm{MHz}$ for the triple layer plate while it is $0.6 \mathrm{MHz}$ for the double layer plate.

Considering the inversion procedure the set of sensitive points used for the double layer plate could not be transferred to the triple layer plate. First of all there are no real gaps along the Alu modes. The crossing points and the slowness minima are affected by the fact that two modes follow one Alu mode. Therefore the detection

of the slowness minima gets more difficult. For the case of the triple layer plate more advanced sensitive points, like asymptotic expressions, have to be found and examined. 


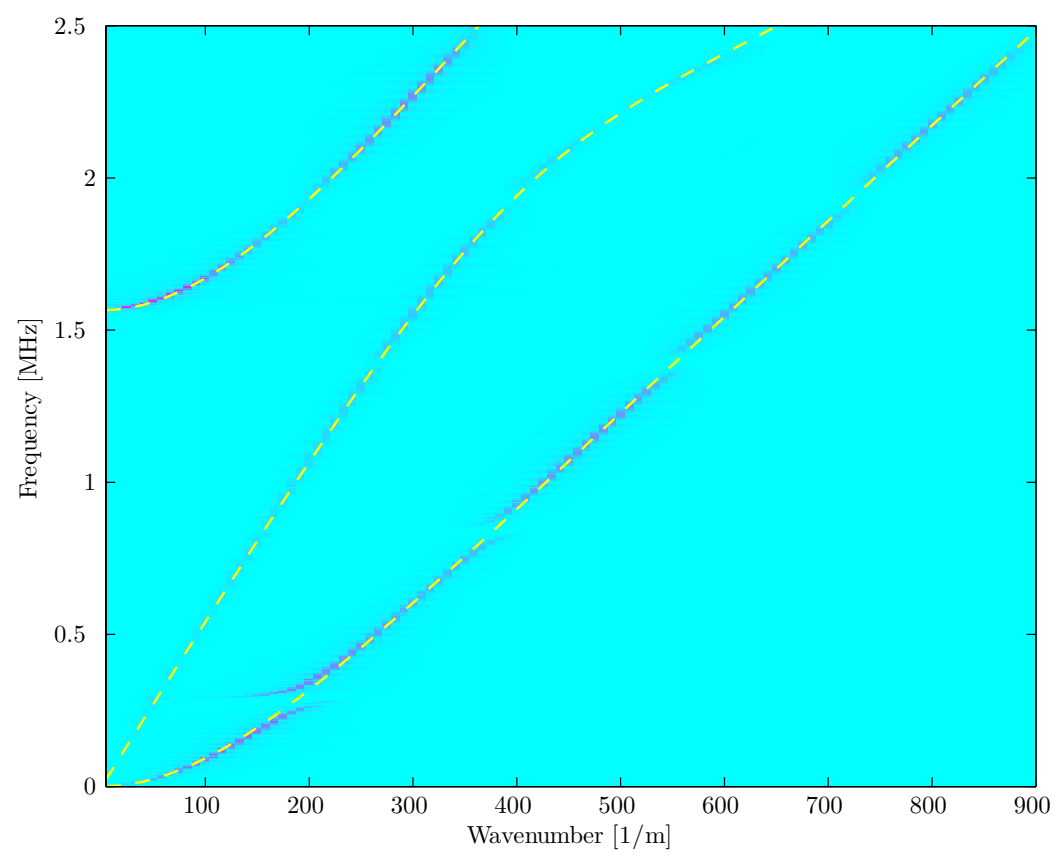

(a) In-plane component

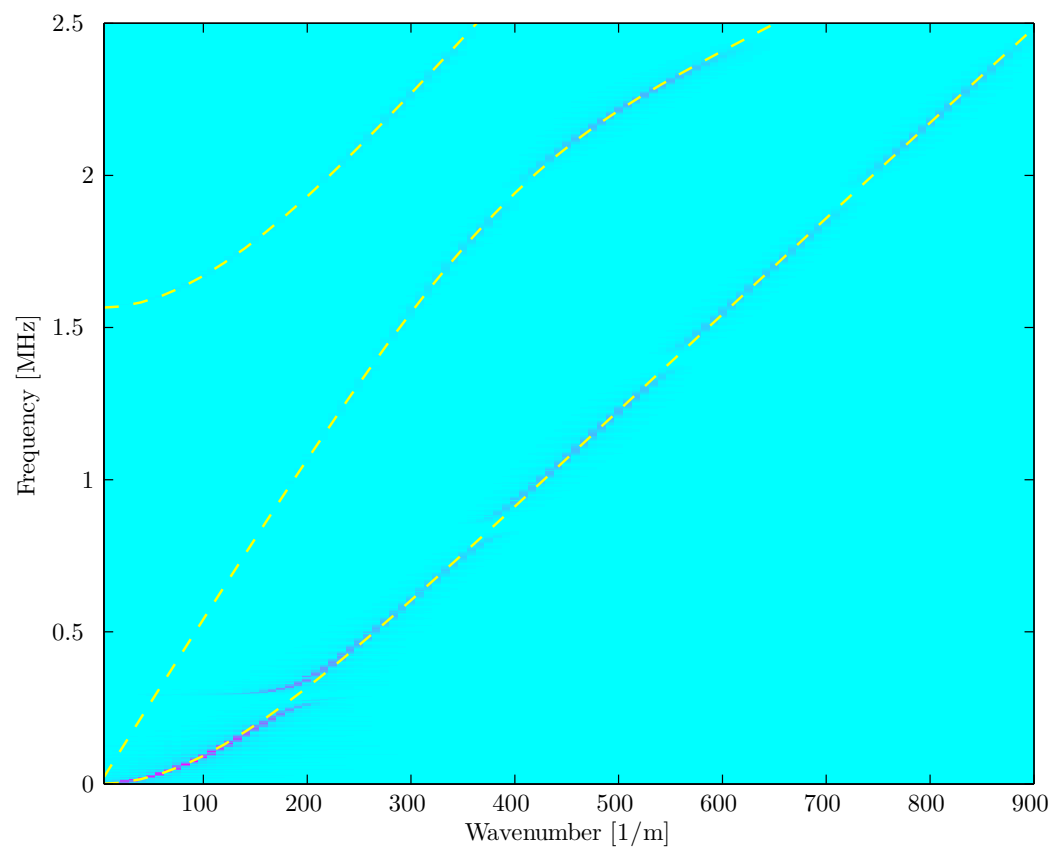

(b) Out-of-plane component

Figure 6.6: Frequency-wavenumber domain after the 2D-FFT of FE results for Alu-Tape1 with Alu modes (GMM) as yellow dashed lines. 


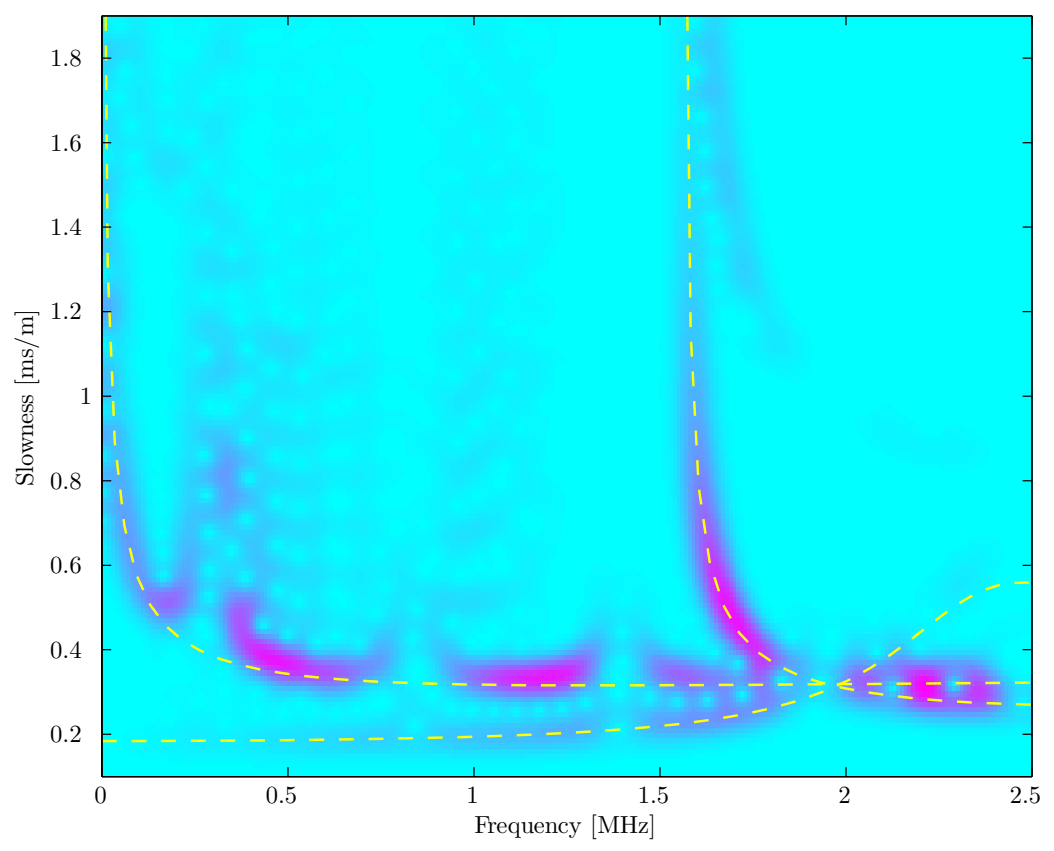

(a) In-plane component

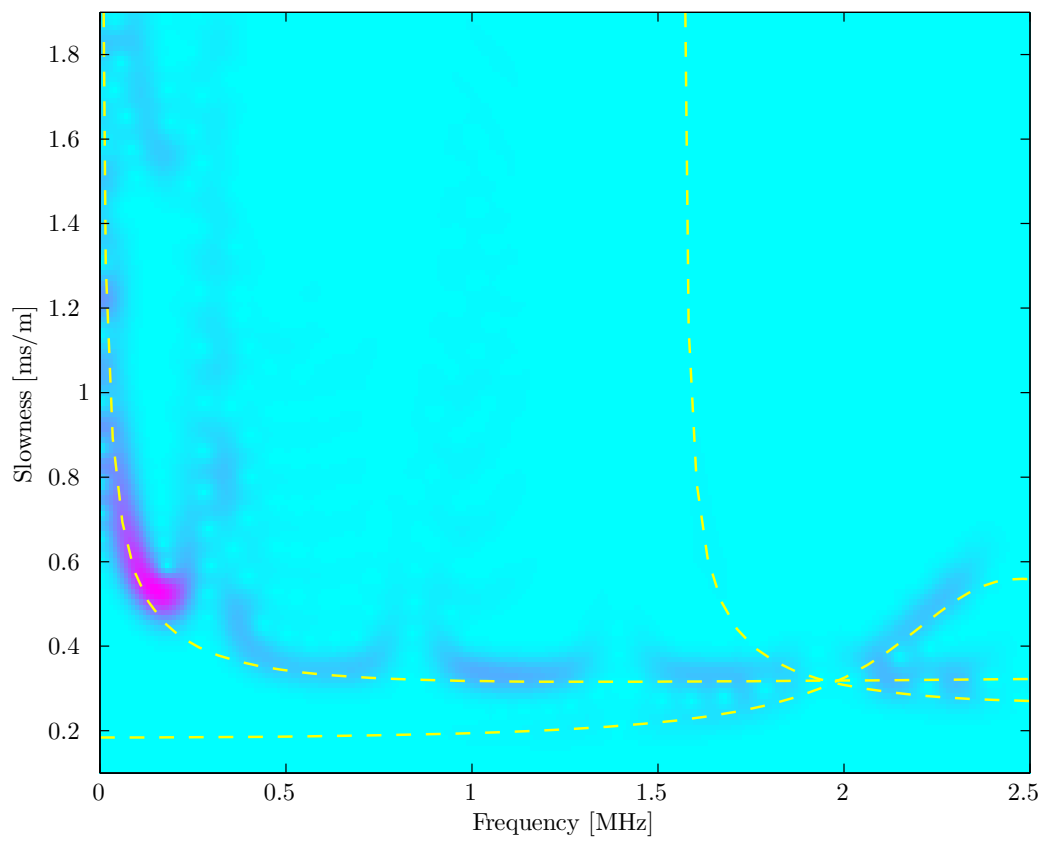

(b) Out-of-plane component

Figure 6.7: Slowness-frequency domain after the STFT of FE results for Alu-Tape1 with Alu modes (GMM) as yellow dashed lines. 


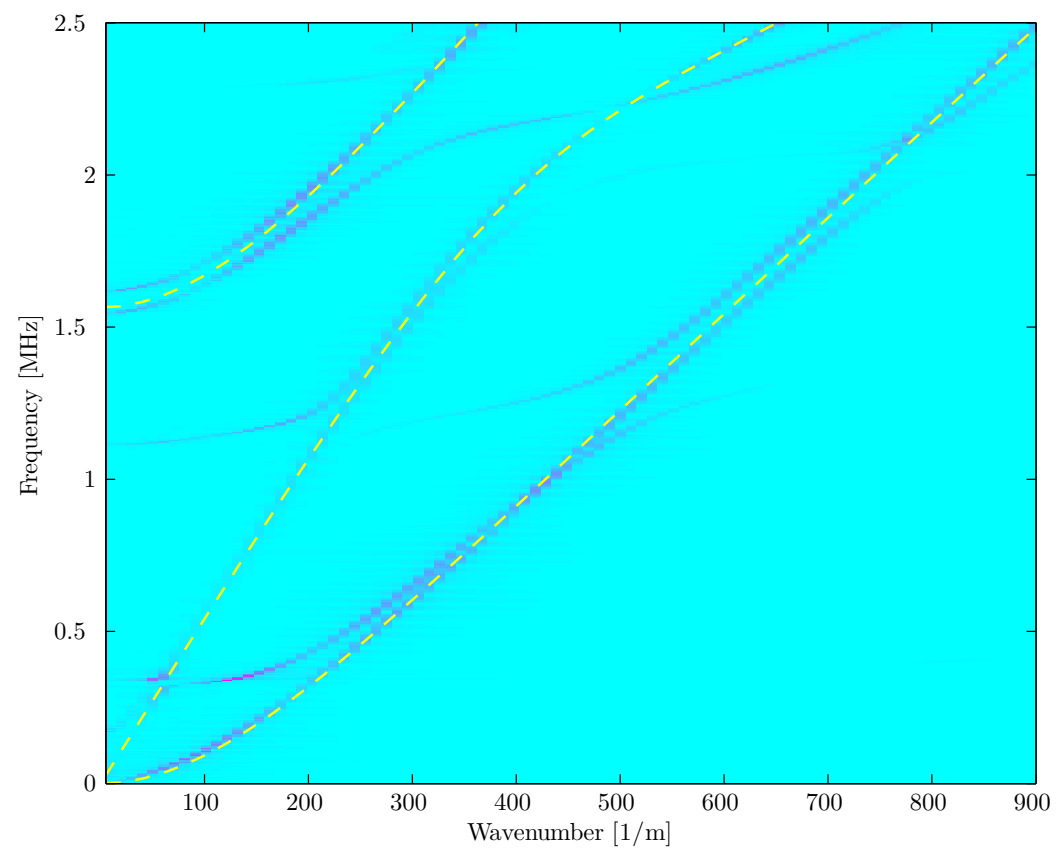

(a) In-plane component

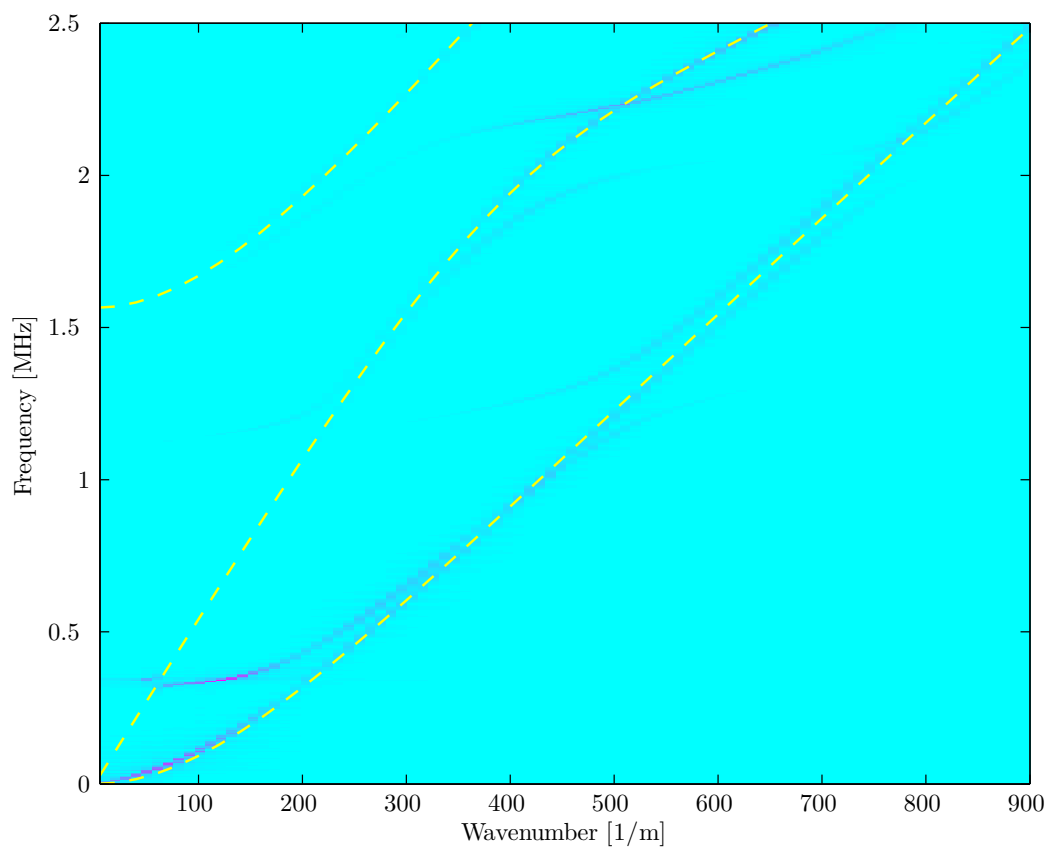

(b) Out-of-plane component

Figure 6.8: Frequency-wavenumber domain after the 2D-FFT of FE results for a triple layered plate (Tape2 in the middle) with Alu modes (GMM) as yellow dashed lines. 


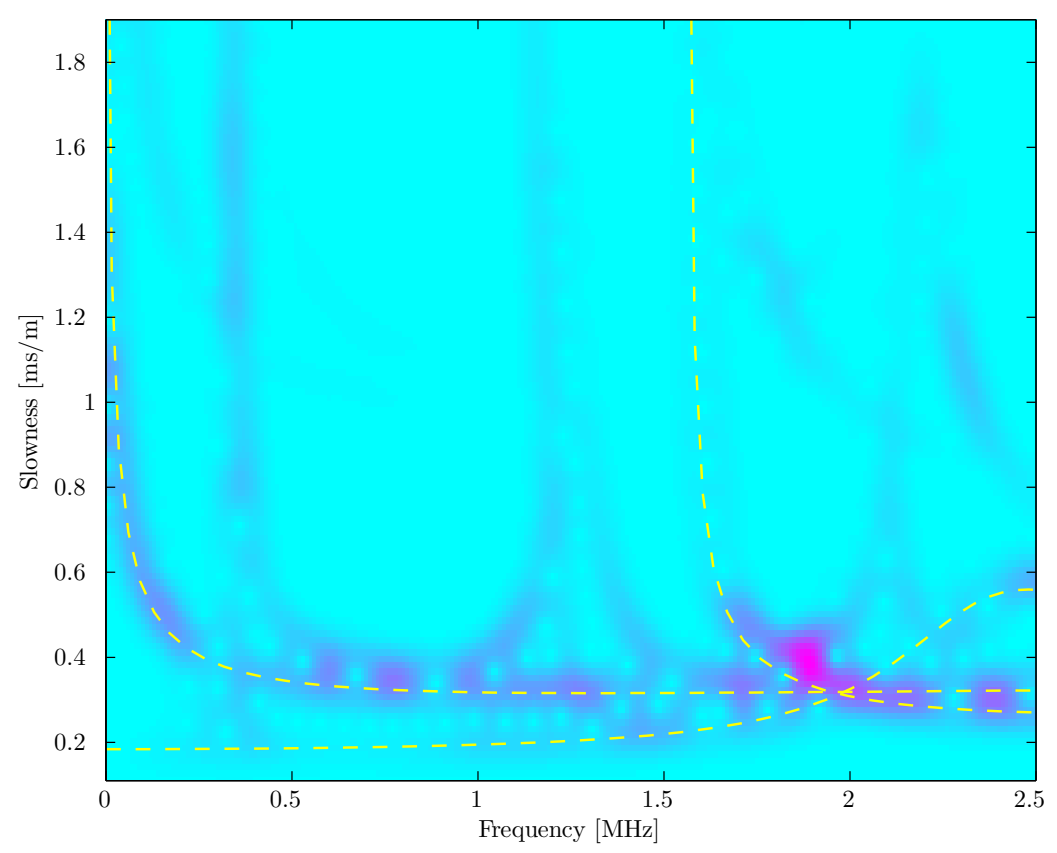

(a) In-plane component

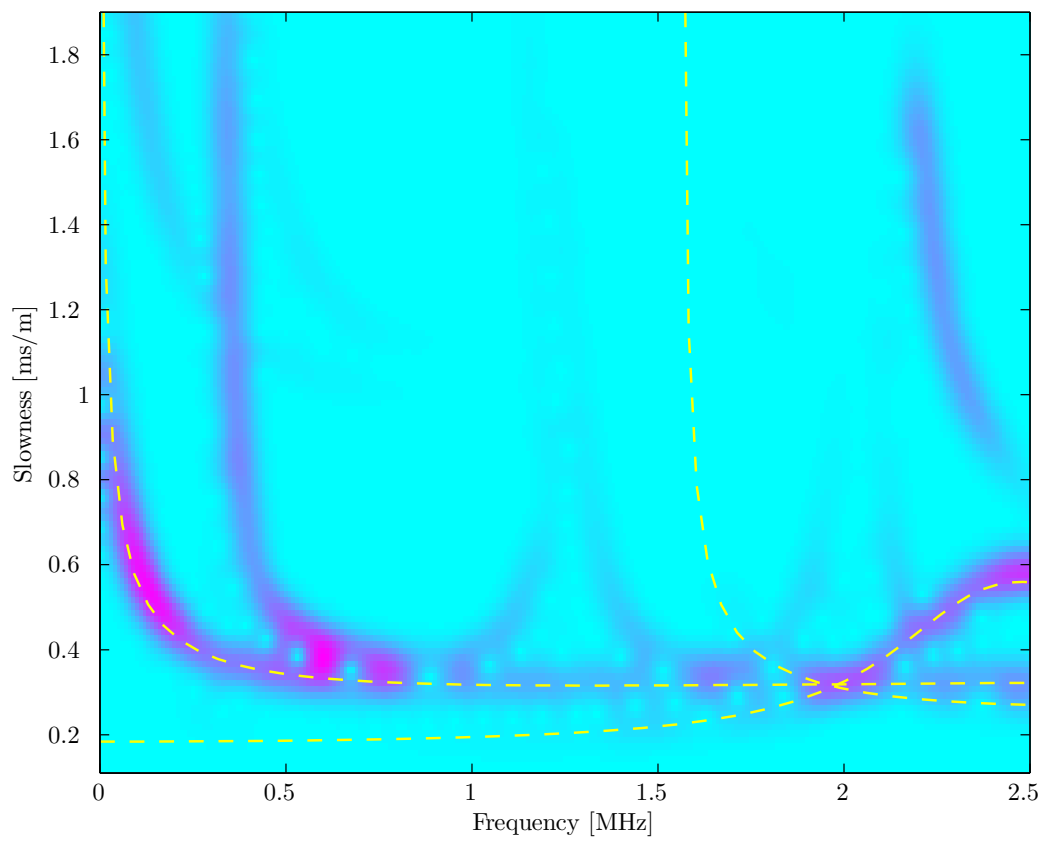

(b) Out-of-plane component

Figure 6.9: Slowness-frequency domain after the STFT of FE results for a triple layered plate (Tape2 in the middle) with Alu modes (GMM) as yellow dashed lines. 


\section{CHAPTER VII}

\section{CONCLUSION}

The goal of this work is to use numerical methods to quantify the material properties of a hidden layer of adhesive bond (tape). The Young's modulus $E_{t}$ of the tape, the Poisson's ratio $\nu_{t}$ of the tape and the tape thickness $h_{t}$ are examined. First, three methods are presented that are able to solve the forward problem. The methods include the global matrix method, the finite element simulation and the waveguide FE method. The result of the forward problem is always a dispersion relation in the form of a frequency-wavenumber or slowness-frequency domain.

In the next step the effects of a parameter change on the dispersion relations are investigated. Based on this and in order to connect the forward and the inverse problems a set of tape parameter sensitive points is identified as input for the inversion procedure. The finite element method and signal processing tools are used to simulate an experiment and create the results on which the input for the inversion procedure is based.

The inversion itself uses the global matrix method to find a set of parameters for which the frequency location of the critical points is equal to the input values. With respect to experimental practices, the capabilities of the inversion process are presented. Depending on the number of transient output signals, it is possible to solve the inverse problem for two or even three parameters. In detail this means that the two parameter optimization based on only one transient FE signal shows reliable results with a deviation sum of only $1.5 \%$ for $E_{t}$ and $\nu_{t}$. The three parameter inversion procedure including $h_{t}$ and based on multiple transient output signals is more complicated, but also shows a deviation sum of only $7.2 \%$ in the best case. 
Further on, an experimental verification of these results as well as the application of this procedure for layered media with more than two layers appears to be feasible. The global matrix method and therefore the inversion process so far is limited to canonical geometries. A larger range of applications could be investigated with a similar time efficiency by using the waveguide FE method in the inversion.

Recommendations for future research would include using a viscoelastic material for the tapes. Further on the suggested procedure could be transferred to plates with more than two layers, which would involve finding new critical points. Another approach would be the implementation of the waveguide FE method in order to solve the inverse problem for more complicated structures. This would also include connecting the FE software with the optimization software. Finally an experimental verification especially for the higher stiffness tape would be able to show the hands-on capabilities of the procedure. 


\section{APPENDIX A}

\section{PARAMETRIC STUDIES (FEM)}
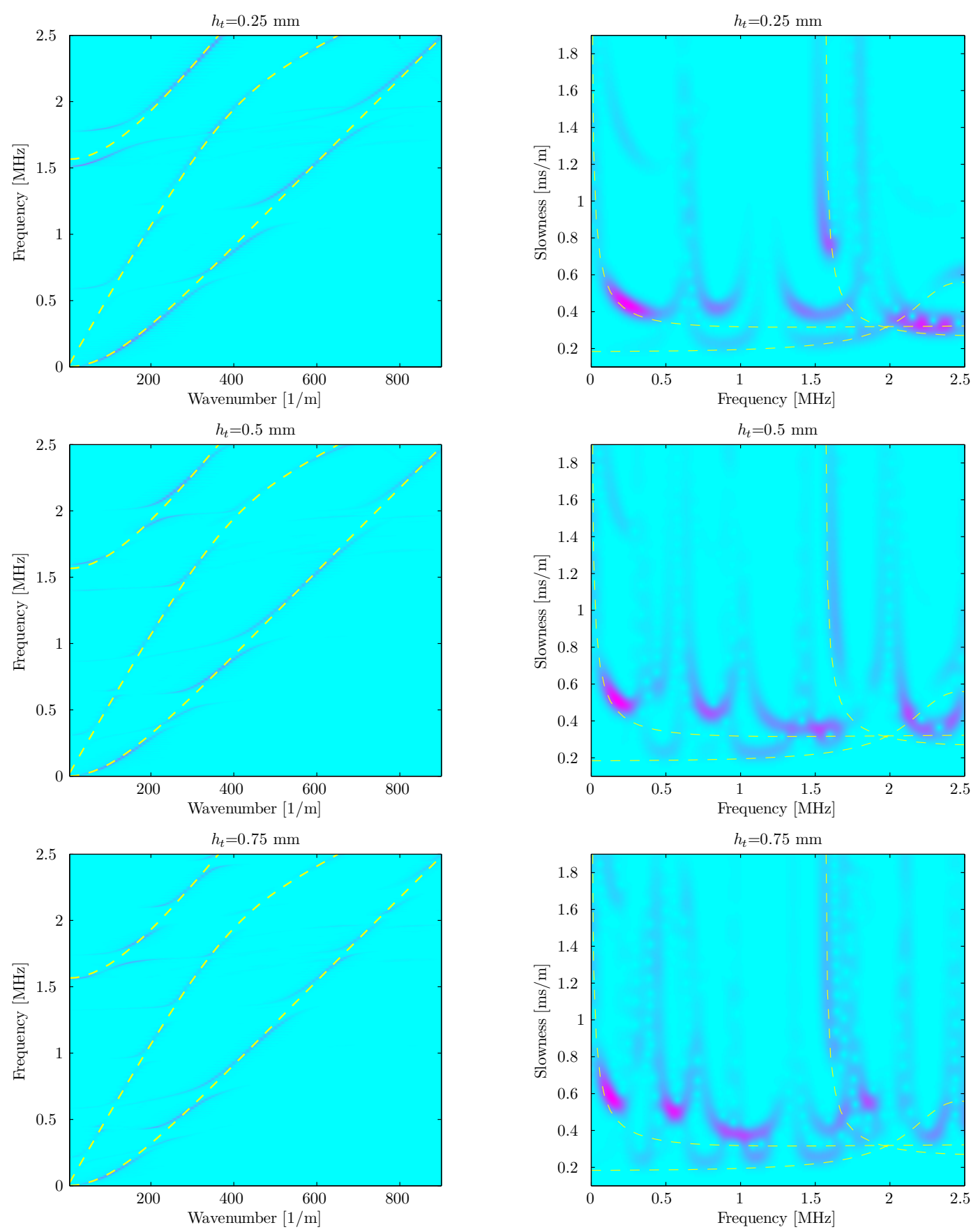

Figure A.1: $h_{t}$ variation for in-plane FEM results of Alu-Tape with Alu modes (GMM) as yellow dashed lines. 

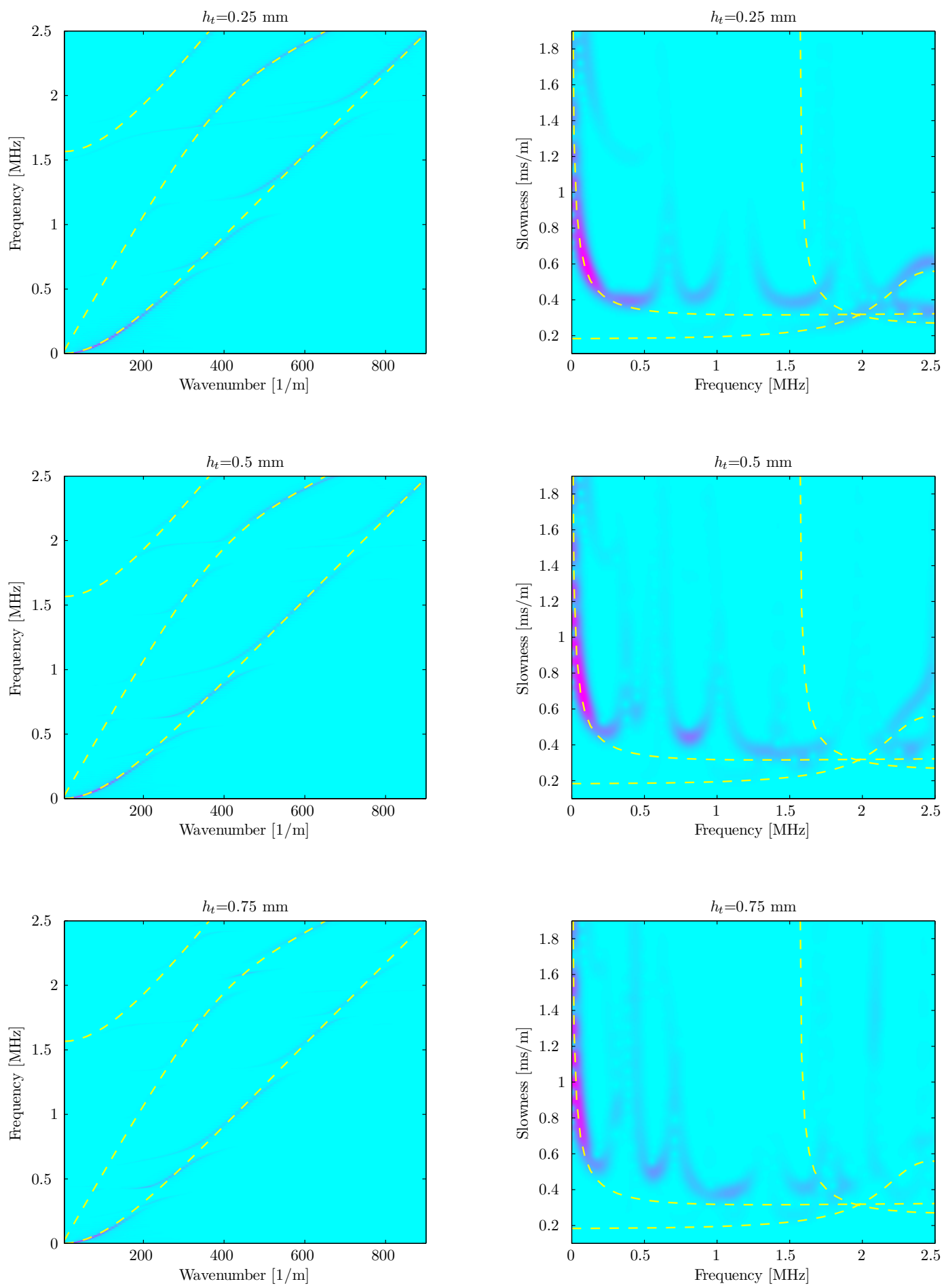

Figure A.2: $h_{t}$ variation for out-of-plane FEM results of Alu-Tape with Alu modes (GMM) as yellow dashed lines. 

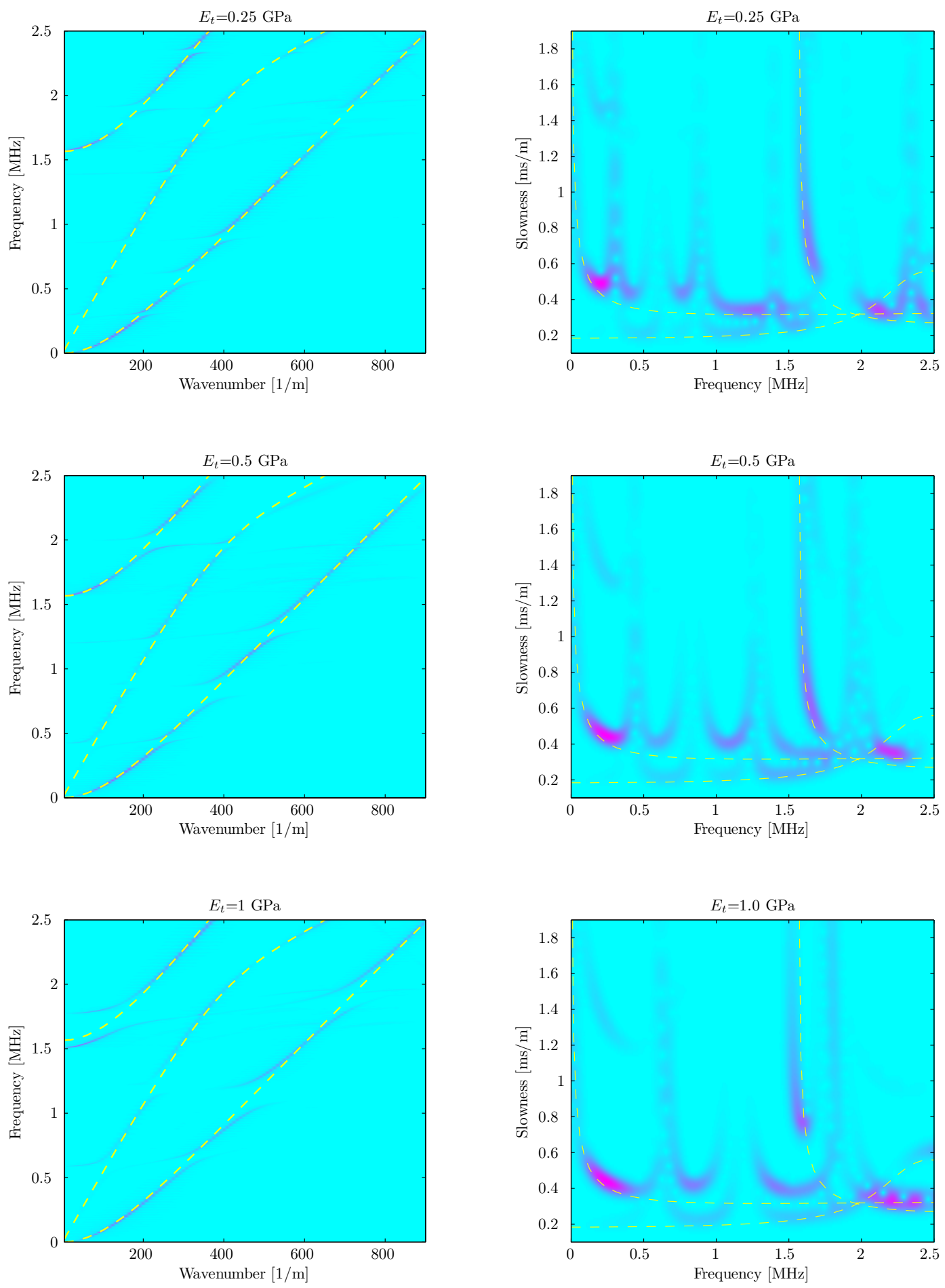

Figure A.3: $E_{t}$ variation for in-plane FEM results of Alu-Tape with Alu modes (GMM) as yellow dashed lines. 

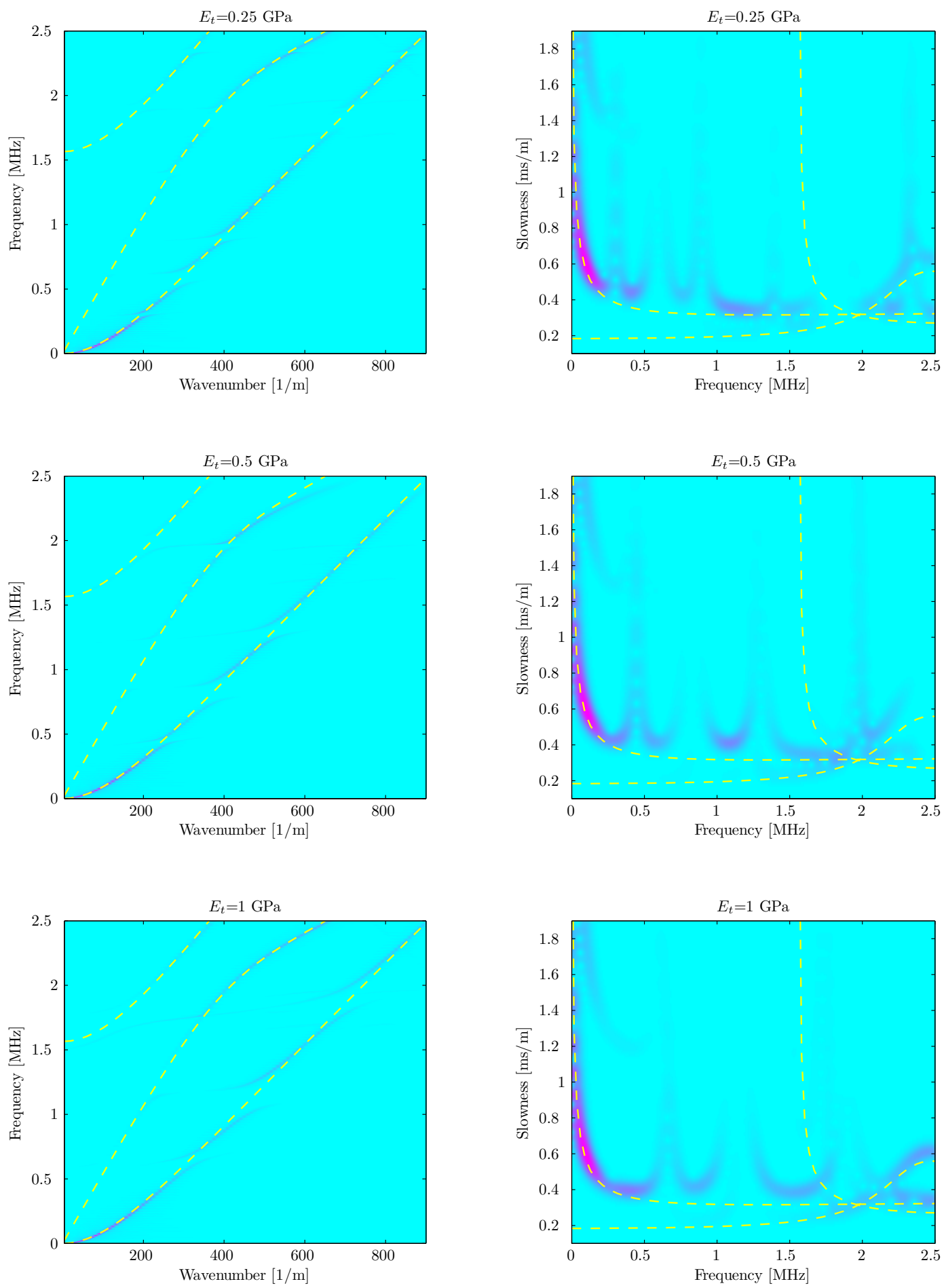

Figure A.4: $E_{t}$ variation for out-of-plane FEM results of Alu-Tape with Alu modes (GMM) as yellow dashed lines. 

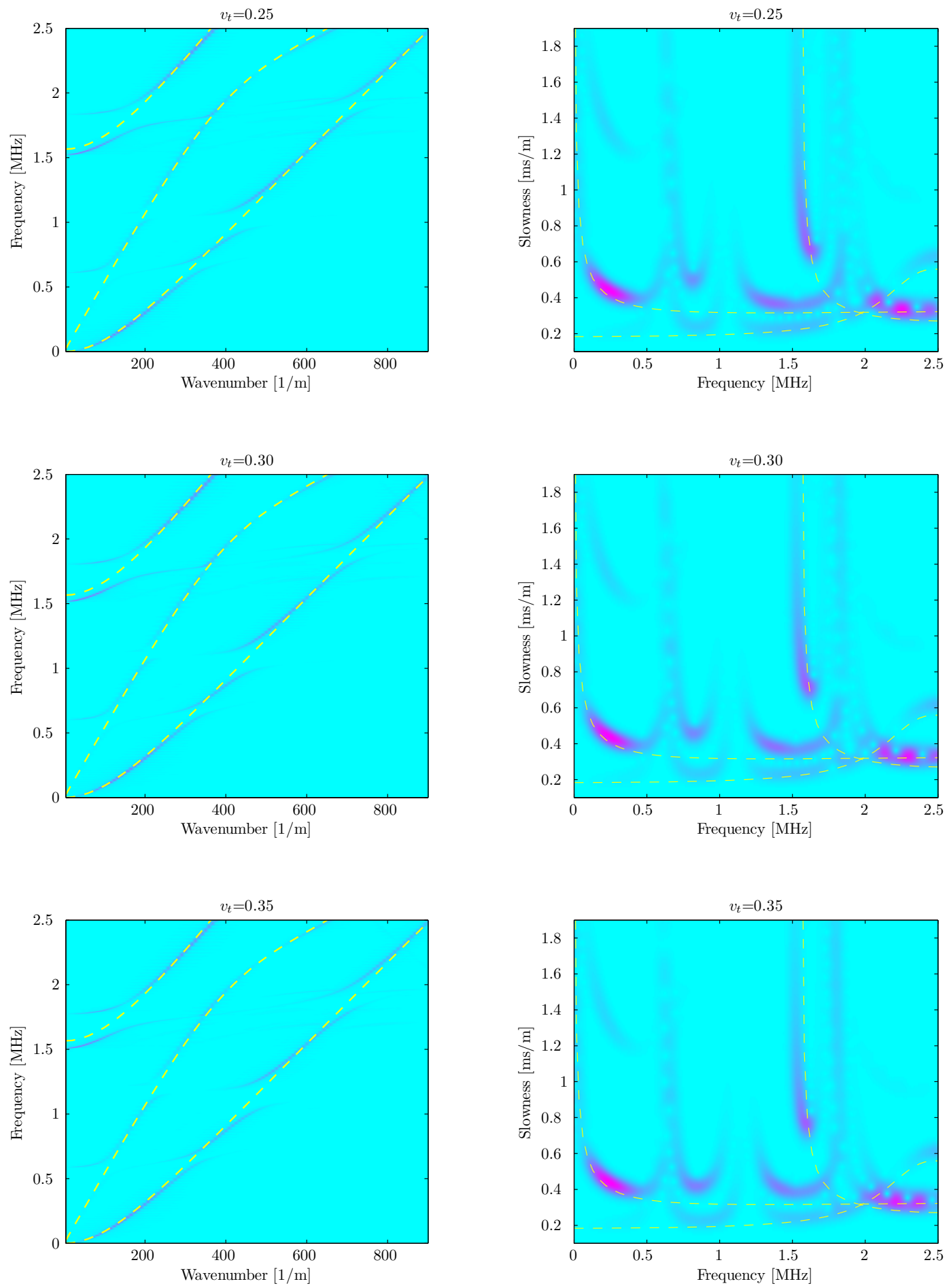

Figure A.5: $\nu_{t}$ variation for in-plane FEM results of Alu-Tape with Alu modes (GMM) as yellow dashed lines. 

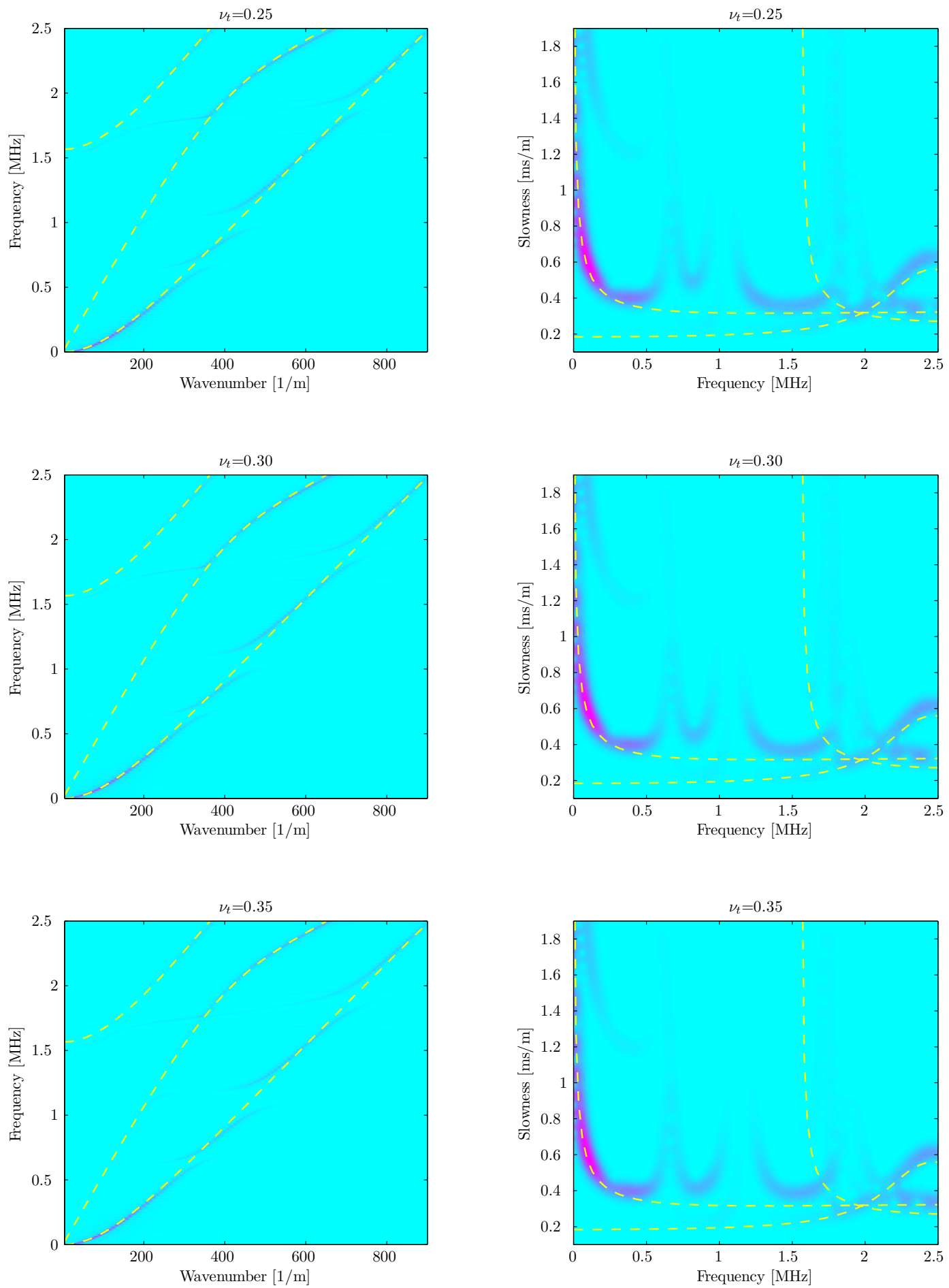

Figure A.6: $\nu_{t}$ variation for out-of-plane FEM results of Alu-Tape with Alu modes (GMM) as yellow dashed lines. 


\section{REFERENCES}

[1] ABAQUS, Inc., ABAQUS Documentation, Version 6.5, 2006.

[2] Achenbach, J. D., Wave Propagation in Elastic Solids. Amsterdam: Elsevier North-Holland, 1984.

[3] Alleyne, D. and Cawley, P., "A two-dimensional Fourier transform method for measurement of propagating multimode signals," J. Acoust. Soc. Am., vol. 89, no. 3, pp. 1159-1168, 1991.

[4] Fletcher, R., Practical methods of optimization. New York: John Wiley and Sons, 2 ed., 1987.

[5] Graff, K. F., Wave Motion in Elastic Solids. Dover Press, Inc., 1975.

[6] Heller, K., Jacobs, L. J., and Qu, J., "Characterization of adhesive bond properties using lamb waves," NDT $\&$ E International, vol. 33, pp. 555-563, 2000.

[7] Herrmann, J., "Numerische Analyse von Dispersionseigenschaften in fluidbefuellten Wellenrohren," in German, Diploma thesis, University of Stuttgart, 2006.

[8] Hosten, B. and Castaings, M., "Surface impedance matrices to model the propagation in multilayered media," Ultrasonics, vol. 41, pp. 501-507, 2003.

[9] Hosten, B. and Castaings, M., "FE modeling of lamb mode diffraction by defects in anisotropic viscoelastic plates," NDT $\&$ E International, vol. 39, pp. 195$204,2006$.

[10] Mace, B. R., Duhamel, D., Brennan, M. J., and Hinke, L., "Finite element prediction of wave motion in structural waveguides," J. Acoust. Soc. Am., vol. 117, pp. 2835-2843, 2005.

[11] Maess, M., Wagner, N., and Gaul, L., "Dispersion curves of fluid filled elastic pipes by standard FE models and eigenpath analysis," Journal of Sound and Vibration, vol. 296, pp. 264-276, 2006.

[12] MathWorks, Inc., Matlab 7.0.4.354(R14) - Optimization Toolbox 3.0.2, 2005.

[13] Mindlin, R., "Waves and vibrations in isotropic, elastic plates," in Structural Mechanics, Proceedings of the first symposium on naval structural mechanics, pp. 199-232, 1958. 
[14] Moser, F., Jacobs, L. J., and Qu, J., "Modeling elastic wave propagation in waveguides with the finite element method," NDT $\&$ E International, vol. 32, no. 4, pp. 225-234, 1999.

[15] Niethammer, M., Jacobs, L., Qu, J., and Jarzynski, J., "Application of time-frequency representations to characterize ultrasonic signals," master's thesis, Georgia Institute of Technology, August 1999.

[16] Oppenheim, A. V. and Schafer, R. W., Discrete-Time Signal Processing. Upper Saddle River, N.J.: Prentice-Hall, 1999.

[17] Pavlakovic, B. and Lowe, M. J. S., Disperse, A system for Generating Dispersion Curves, Version 2.0, 2000.

[18] Rose, J. L., Ultrasonic Waves in Solid Media. Cambridge University Press, 1999.

[19] Rose, J. L., Zhu, W., and ZAidi, M., "Ultrasonic NDT of titanium diffusion bonding with guided waves," Materials Evaluation, vol. 56, no. 4, pp. 535-539, 1998.

[20] Seifried, R., Jacobs, L. J., and Qu, J., "Propagation of guided waves in adhesive bonded components," NDT $\mathscr{E}$ E International, vol. 35, pp. 317-328, 2002.

[21] Valle, C., Guided circumferential waves in annular structures. PhD thesis, Georgia Institute of Technology, Atlanta, Georgia, 1999.

[22] Valle, C., Niethammer, M., Qu, J., and Jacobs, L. J., "Chrack characterization using guided circumferential waves," J. Acoust. Soc. Am., vol. 110, pp. 1282-1290, 2001.

[23] Zienkiewicz, O. C. and Taylor, R. L., The Finite Element Method. Oxford: Butterworth-Heinemann, 2000. 